\title{
Superconvergence of ultra-weak discontinuous Galerkin methods for the linear Schrödinger equation in one dimension
}

\author{
Anqi Chen * $\quad$ Yingda Cheng ${ }^{\dagger} \quad$ Yong Liu $^{\ddagger} \quad$ Mengping Zhang $§$ \\ May 21, 2019
}

\begin{abstract}
We analyze the superconvergence properties of ultra-weak discontinuous Galerkin (UWDG) methods with various choices of flux parameters for one-dimensional linear Schrödinger equation. In our previous work [10], stability and optimal convergence rate are established for a large class of flux parameters. Depending on the flux choices and if the polynomial degree $k$ is even or odd, in this paper, we prove $2 k$ or $(2 k-1)$-th order superconvergence rate for cell averages and numerical flux of the function, as well as $(2 k-1)$ or $(2 k-2)$-th order for numerical flux of the derivative. In addition, we prove superconvergence of $(k+2)$ or $(k+3)$-th order of the DG solution towards a special projection. At a class of special points, the function values and the first and second order derivatives of the DG solution are superconvergent with order $k+2, k+1, k$, respectively. The proof relies on the correction function techniques initiated in [8], and applied to [6] for direct DG (DDG) methods for diffusion problems. Compared with [6], Schrödinger equation poses unique challenges for superconvergence proof because of the lack of the dissipation mechanism from the equation. One major highlight of our proof is that we introduce specially chosen test functions in the error equation and show the superconvergence of the second derivative and jump across the cell interfaces of the difference between numerical solution and projected exact solution. This technique was originally proposed in [12] and is essential to elevate the convergence order for our analysis. Finally, by negative norm estimates, we apply the post-processing technique and show that the accuracy of our scheme can be enhanced to order $2 k$. Theoretical results are verified by numerical experiments.
\end{abstract}

Keywords. Ultra-weak discontinuous Galerkin method, superconvergence, post-processing, projection, one-dimensional Schrödinger equation.

\footnotetext{
*Department of Mathematics, Michigan State University, East Lansing, MI 48824 U.S.A. chenanq3@msu. edu.

${ }^{\dagger}$ Department of Mathematics, Department of Computational Mathematics, Science and Engineering, Michigan State University, East Lansing, MI 48824, USA. E-mail: ycheng@msu.edu. Research is supported by NSF grants DMS-1453661 and DMS-1720023.

$\ddagger$ School of Mathematical Sciences, University of Science and Technology of China, Hefei, Anhui 230026 People's Republic of China. yong123@mail.ustc.edu.cn.

${ }^{\S}$ School of Mathematical Sciences, University of Science and Technology of China, Hefei, Anhui, 230026 People's Republic of China. mpzhang@ustc.edu.cn. Research supported by NSFC grant 11871448.
} 


\section{Introduction}

Discontinuous Galerkin (DG) methods belong to a class of finite element methods using discontinuous piecewise function space for test functions and numerical solution. The first DG method was introduced by Reed and Hill in [21] for solving neutron transport problems. A major development of DG methods is the Runge-Kutta DG (RKDG) framework introduced for solving hyperbolic conservation laws in a series of papers, see [14] for a review. Because of the completely discontinuous basis, DG methods have several attractive properties. It can be used on many types of meshes, even those with hanging nodes. The methods can be designed with $h-p$ adaptivity and very high parallel efficiency.

We are interested in solving the following linear Schrödinger equations by DG methods.

$$
\begin{aligned}
& i u_{t}+u_{x x}=0, \quad(x, t) \in I \times\left(0, T_{e}\right], \\
& u(x, 0)=u_{0}(x),
\end{aligned}
$$

where $I=[a, b]$ and periodic boundary condition. Various types of DG schemes for discretizing the second order spatial derivatives have been used to compute (1), including the local DG (LDG) method [25, 26, 17] and the direct DG (DDG) methods [18]. This paper will focus on the ultra-weak DG (UWDG) methods, which can be traced backed to [9], and refer to those DG methods [24] that rely on repeatedly applying integration by parts so all the spatial derivatives are shifted from the solution to the test function in the weak formulations [11, 3]. In our previous work [10], UWDG methods were studied, and a systematic choice of flux parameters were made to guarantee stability or energy conservation property of the scheme. Moreover, using projection techniques, convergence results of the UWDG method for the one-dimensional nonlinear Schrödinger equation were established. It was shown that a wide range of flux parameter choices can yield optimally convergent scheme. In this work, we continue the research and investigate superconvergence of the UWDG scheme.

The study of superconvergence is of importance because a posteriori error estimates can be derived guiding adaptive calculations. For superconvergence of DG methods, many results exist in the literature. We refer the readers to [2, 1] for ordinary differential equation results. In [12, Cheng and Shu proved that the DG and LDG solutions are $(k+3 / 2)$-th order superconvergent towards projections of exact solutions of hyperbolic conservation laws and convection-diffusion equations using specially designed test functions when piecewise polynomials of degree $k$ are used. For linear hyperbolic problems, in [27], Yang and Shu proved that, under suitable initial discretization, the DG solutions of linear hyperbolic systems are convergent with optimal $(k+2)$-th order at Radau points. More recently, in [8], Cao et al proved the $(2 k+1)$-th superconvergence rate for cell average and DG numerical fluxes by introducing a locally defined correction function. The correction function also helps simplify the proof for point wise $(k+2)$-th superconvergence rate at Radau points and prove the derivative of DG solution has $(k+1)$-th superconvergence rate at so-called "left Radau" points. Then this technique has been extended to prove the superconvergence of DG solutions for linear and nonlinear hyperbolic PDEs in [7, 5, DDG method for convection diffusion equations [6] and LDG method for linear Schrödinger equations [28]. Overall, for equations with higher order spatial derivatives, the same type of correction functions can be used for the LDG method which is based on a reformulation into a first order system of equations. For DDG method, new correction functions are needed treating the second order derivative directly [6]. 
Another type of superconvergence of DG methods is achieved by postprocessing the solution by convolution with a kernel function, which is a linear combination of B-spline functions. For linear hyperbolic systems, [13] provided a framework for constructing such postprocessor and proving the superconvergence of the postprocessed DG solutions. Through the analysis of negative norm estimates and divided difference estimates, they showed that the postprocessed solution is superconvergent at a rate of $2 k+1$. More recently, in [16, 19] the analysis are extended to scalar nonlinear hyperbolic equations.

In this work, we aim at the study of superconvergence of the UWDG methods for (1) with scale invariant flux parameters. Such choice include all commonly used fluxes, e.g. alternating, central, DDG and interior penalty DG (IPDG) fluxes. Depending on the flux choices and the evenness of oddness of the polynomial degree $k$, we obtain $2 k$ or $(2 k-1)$ th order superconvergence rate for cell averages and numerical flux of the function, as well as $(2 k-1)$ or $(2 k-2)$-th order for numerical flux of derivative. The proof relies on the correction function techniques for second order derivatives applied to [6] for DDG methods for diffusion problems. This correction function also enable us to prove the UWDG solution is superconvergent with a rate of $k+3$ to the special projection $P_{h}^{\star}$ we introduced in [10] if $k \geq 3$. We show that the function values and the first and second order derivatives of the DG solution are superconvergent with order $k+2, k+1, k$, respectively, at interior points whose locations are determined by roots of certain polynomials associated with the flux parameters. We want to emphasize that our approach is related but different from the superconvergence proof in [6] for diffusion equations, mainly because there is no dissipation mechanism in the Schrödinger equation. Therefore, when $k$ is even, there is some additional terms in the error estimates that cannot be bounded. To overcome this difficulty, we take specially chosen test functions in the error equation and show the superconvergence of some intermediate quantities. This technique was originally proposed in [12] and is essential to elevate the convergence order for our scheme. For the postprocessed UWDG solution, we introduce a dual problem and prove $(2 k)$-th order negative norm estimate. The order is also one order less than that in hyperbolic equations, and again, due to the ultra-weak formulation which has boundary term of the product of derivatives and function values. With the negative norm estimates and divided difference estimates, we prove the $(2 k)$-th order superconvergence rate for the postprocessed solution.

The rest of the paper is organized as follows. In Section 2, we recall the UWDG scheme for linear Schrödinger equations and some properties of the spatial discretization. In Section 3. we define notations and projections. Section 4 contains the main results of the paper, superconvergence of the UWDG solution in various measures. In Section 5, we provide numerical tests verifying theoretical results. Finally, we conclude in Section 6. Some technical proof is provided in the Appendix.

\section{Numerical Scheme}

We first define notations of the mesh and finite element solution space. For interval $I=[a, b]$, the usual DG mesh $\mathcal{I}_{N}$ and the index set $\mathbb{Z}_{N}=\{1,2, \cdots, N\}$ is defined as:

$$
a=x_{\frac{1}{2}}<x_{\frac{3}{2}}<\cdots<x_{N+\frac{1}{2}}=b, \quad I_{j}=\left(x_{j-\frac{1}{2}}, x_{j+\frac{1}{2}}\right), x_{j}=\frac{1}{2}\left(x_{j-\frac{1}{2}}+x_{j+\frac{1}{2}}\right), j \in \mathbb{Z}_{N}
$$


and

$$
h_{j}=x_{j+\frac{1}{2}}-x_{j-\frac{1}{2}}, \quad h=\max _{j \in \mathbb{Z}_{N}} h_{j},
$$

with mesh regularity requirement $\frac{h}{\min h_{j}}<\sigma, \sigma$ is fixed during mesh refinement. The approximation space, which is a piecewise complex polynomial space on $\mathcal{I}_{N}$ is defined as:

$$
V_{h}^{k}=\left\{v_{h}:\left.v_{h}\right|_{I_{j}} \in P_{c}^{k}\left(I_{j}\right), I_{j} \in \mathcal{I}_{N}\right\}
$$

where $P_{c}^{k}\left(I_{j}\right)$ is the space of complex polynomials of degree up to $k$ on cell $I_{j}$. For a function $v_{h} \in V_{h}^{k}$, we use $\left(v_{h}\right)_{j-\frac{1}{2}}^{-}$and $\left(v_{h}\right)_{j-\frac{1}{2}}^{+}$to denote the value of $v_{h}$ at $x_{j-\frac{1}{2}}$ from the left cell $I_{j-1}$ and the right cell $I_{j}$ respectively. The jump and average values are defined as $\left[v_{h}\right]=v_{h}^{+}-v_{h}^{-}$ and $\left\{v_{h}\right\}=\frac{1}{2}\left(v_{h}^{+}+v_{h}^{-}\right)$at cell interfaces.

Throughout the paper, we use the standard Sobolev norm notations $\|\cdot\|_{W^{s, p}(I)}$ and broken Sobolev space on mesh $\mathcal{I}_{N}$. We denote $\|v\|_{H^{s}\left(\mathcal{I}_{N}\right)}^{2}=\sum_{j=1}^{N}\|v\|_{H^{s}\left(I_{j}\right)}^{2}$ and $\|v\|_{W^{s, \infty}\left(\mathcal{I}_{N}\right)}=$ $\max _{j}\|v\|_{W^{s, \infty}\left(I_{j}\right)}$. In Section 4.3, we consider negative norms and the definition is $\|v\|_{H^{-l}(I)}=$ $\sup _{\Phi \in \mathcal{C}_{0}^{\infty}(I)} \frac{\int_{I} v(x) \Phi(x) d x}{\|\Phi\|_{H^{l}(I)}}$. Additionally, we denote by $\|v\|_{L^{2}\left(\partial \mathcal{I}_{N}\right)}$ the broken $L^{2}$ norm on cell interfaces, i.e., $\|v\|_{L^{2}\left(\partial \mathcal{I}_{N}\right)}^{2}=\sum_{j=1}^{N}\|v\|_{L^{2}\left(\partial I_{j}\right)}^{2}$, where $\|v\|_{L^{2}\left(\partial I_{j}\right)}^{2}=\left(v_{x_{j+\frac{1}{2}}}^{-}\right)^{2}+\left(v_{x_{j-\frac{1}{2}}}^{+}\right)^{2}$. We also denote $\|\cdot\|=\|\cdot\|_{L^{2}(I)}=\|\cdot\|_{L^{2}\left(\mathcal{I}_{N}\right)}$ to shorten the notation. Lastly, we recall inverse inequalities

$$
\begin{array}{r}
\left\|\left(v_{h}\right)_{x}\right\|_{L^{2}\left(\mathcal{I}_{j}\right)} \leq C h_{j}^{-1}\left\|v_{h}\right\|_{L^{2}\left(\mathcal{I}_{j}\right)}, \quad\left\|v_{h}\right\|_{L^{2}\left(\partial \mathcal{I}_{j}\right)} \leq C h^{-\frac{1}{2}}\left\|v_{h}\right\|_{L^{2}\left(\mathcal{I}_{j}\right)}, \\
\left\|v_{h}\right\|_{L^{\infty}\left(\mathcal{I}_{j}\right)} \leq C h^{-\frac{1}{2}}\left\|v_{h}\right\|_{L^{2}\left(\mathcal{I}_{j}\right)}, \quad \forall v_{h} \in V_{h}^{k},
\end{array}
$$

and trace inequalities

$$
\|v\|_{L^{2}\left(\partial I_{j}\right)}^{2} \leq C h_{j}^{-1}\|v\|_{L^{2}\left(I_{j}\right)}^{2},
$$

where, here and below $C$ is a constant independent of the function $u$ and the mesh size $h$.

The semi-discrete UWDG scheme formulated in [10] is defined as follows: we solve for the unique function $u_{h}=u_{h}(t) \in V_{h}^{k}, k \geq 1, t \in\left(0, T_{e}\right]$, such that

$$
a_{j}\left(u_{h}, v_{h}\right)=0, \quad \forall j \in \mathbb{Z}_{N}
$$

holds for all $v_{h} \in V_{h}^{k}$, where

$$
a_{j}\left(u_{h}, v_{h}\right)=\int_{I_{j}}\left(u_{h}\right)_{t} v_{h} d x-i A_{j}\left(u_{h}, v_{h}\right)
$$

with $A_{j}\left(u_{h}, v_{h}\right)=\int_{I_{j}} u_{h}\left(v_{h}\right)_{x x} d x-\left.\hat{u}_{h}\left(v_{h}\right)_{x}^{-}\right|_{j+\frac{1}{2}}+\left.\hat{u}_{h}\left(v_{h}\right)_{x}^{+}\right|_{j-\frac{1}{2}}+\left.\widetilde{\left(u_{h}\right)_{x}} v_{h}^{-}\right|_{j+\frac{1}{2}}-\left.\widetilde{\left(u_{h}\right)_{x}} v_{h}^{+}\right|_{j-\frac{1}{2}}$ as the UWDG spatial discretization for the second order derivative term. The "hat" and "tilde" terms are the numerical fluxes for $u$ and $u_{x}$ at cell boundaries, which are single valued functions defined as:

$$
\begin{array}{rlr}
\hat{u}_{h} & =\left\{u_{h}\right\}-\alpha_{1}\left[u_{h}\right]+\beta_{2}\left[\left(u_{h}\right)_{x}\right], \quad \alpha_{1}, \beta_{2} \in \mathbb{R} . \\
\widetilde{\left(u_{h}\right)_{x}} & =\left\{\left(u_{h}\right)_{x}\right\}+\alpha_{1}\left[\left(u_{h}\right)_{x}\right]+\beta_{1}\left[u_{h}\right], \quad \beta_{1} \in \mathbb{R},
\end{array}
$$


where $\alpha_{1}, \beta_{1}, \beta_{2}$ are prescribed parameters that may have $h$ dependence. Note that we can rewrite the flux definition above in a matrix form

$$
\left[\frac{\hat{u}_{h}}{\left(u_{h}\right)_{x}}\right]=G\left[\begin{array}{c}
u_{h}^{-} \\
\left(u_{h}\right)_{x}^{-}
\end{array}\right]+H\left[\begin{array}{c}
u_{h}^{+} \\
\left(u_{h}\right)_{x}^{+}
\end{array}\right], \quad G=\left[\begin{array}{cc}
\frac{1}{2}+\alpha_{1} & -\beta_{2} \\
-\beta_{1} & \frac{1}{2}-\alpha_{1}
\end{array}\right], H=I_{2}-G=\left[\begin{array}{cc}
\frac{1}{2}-\alpha_{1} & \beta_{2} \\
\beta_{1} & \frac{1}{2}+\alpha_{1}
\end{array}\right],
$$

where $I_{2}$ denotes the $2 \times 2$ identity matrix. Some commonly used fluxes take the following choices of parameters.

- central flux, $\alpha_{1}=\beta_{1}=\beta_{2}=0$;

- alternating flux, $\alpha_{1}= \pm \frac{1}{2}, \beta_{1}=\beta_{2}=0$;

- IPDG like flux, $\alpha_{1}=\beta_{2}=0, \beta_{1}=\tilde{\beta}_{1} h^{-1}$;

- DDG like flux, $\alpha_{1}=\tilde{\alpha}_{1}, \beta_{2}=0, \beta_{1}=\tilde{\beta}_{1} h^{-1}$;

- more generally, any scale invariant flux, $\alpha_{1}=\tilde{\alpha_{1}}, \beta_{1}=\tilde{\beta}_{1} h^{-1}, \beta_{2}=\tilde{\beta}_{2} h$;

where $\tilde{\alpha_{1}}, \tilde{\beta}_{1}, \tilde{\beta}_{2}$ are prescribed constants independent of mesh size. For simplicity, in this paper we will only consider scale invariant flux choices. We now introduce

$$
\begin{aligned}
& a\left(u_{h}, v_{h}\right)=\sum_{j=1}^{N} a_{j}\left(u_{h}, v_{h}\right), \\
& A\left(u_{h}, v_{h}\right)=\sum_{j=1}^{N} A_{j}\left(u_{h}, v_{h}\right)=\int_{I} u_{h}\left(v_{h}\right)_{x x} d x+\left.\sum_{j=1}^{N}\left(\hat{u}_{h}\left[\left(v_{h}\right)_{x}\right]-\widetilde{\left(u_{h}\right)_{x}}\left[v_{h}\right]\right)\right|_{j+\frac{1}{2}} .
\end{aligned}
$$

Clearly, the scheme boils down to requiring $a\left(u_{h}, v_{h}\right)=0, \forall v_{h} \in V_{h}^{k}$.

The following lemma shows the symmetry property of $A(\cdot, \cdot)$.

Lemma 2.1 (Symmetry of $A(\cdot, \cdot)$ ). For $u, v \in H^{2}\left(\mathcal{I}_{N}\right)$ satisfying periodic boundary condition, we have $A(u, v)=A(v, u)$. Furthermore, $A(v, \bar{v}) \in \mathbb{R}$. Here and in what follows, the overline means complex conjugate.

Proof. From integration by parts, we have

$$
A(u, v)=-\int_{I} u_{x} v_{x} d x+\left.\sum_{j=1}^{N}\left(\hat{u}\left[v_{x}\right]-\left[u v_{x}\right]-\widetilde{u_{x}}[v]\right)\right|_{j+\frac{1}{2}} .
$$

Similarly, $A(v, u)=-\int_{I} u_{x} v_{x} d x+\left.\sum_{j=1}^{N}\left(\hat{v}\left[u_{x}\right]-\left[v u_{x}\right]-\widetilde{v_{x}}[u]\right)\right|_{j+\frac{1}{2}}$. Plugging in the definition of the numerical fluxes in (5), we have at $x_{j+\frac{1}{2}}, \forall j \in \mathbb{Z}_{N}$

$$
\begin{aligned}
\hat{u}\left[v_{x}\right]-\left[u v_{x}\right]-\widetilde{u_{x}}[v] & =\left(\{u\}-\alpha_{1}[u]+\beta_{2}\left[u_{x}\right]\right)\left[v_{x}\right]-\left(\{u\}\left[v_{x}\right]+[u]\left\{v_{x}\right\}\right) \\
& -\left(\left\{u_{x}\right\}+\alpha_{1}\left[u_{x}\right]+\beta_{1}[u]\right)[v] \\
& =\left[u_{x}\right]\left(\{v\}-\alpha_{1}[v]+\beta_{2}\left[v_{x}\right]\right)-[u]\left(\left\{v_{x}\right\}+\alpha_{1}\left[v_{x}\right]+\beta_{1}[v]\right) \\
& -\left(\left[u_{x}\right]\{v\}+\left\{u_{x}\right\}[v]\right) \\
& =\left[u_{x}\right] \hat{v}-[u] \widetilde{v}_{x}-\left[u_{x} v\right],
\end{aligned}
$$


then the proof for $A(u, v)=A(v, u)$ is complete. It follows that $A(v, \bar{v})-\overline{A(v, \bar{v})}=A(v, \bar{v})-$ $A(\bar{v}, v)=0$, which implies $A(v, \bar{v}) \in \mathbb{R}$.

In our previous work [10], we proved that our semi-discrete scheme is energy conservative, which is a direct result of the lemma above:

$$
0=a\left(u_{h}, \overline{u_{h}}\right)+\overline{a\left(u_{h}, \overline{u_{h}}\right)}=\frac{d}{d t}\left\|u_{h}\right\|^{2}-i A\left(u_{h}, \bar{u}_{h}\right)+i A\left(u_{h}, \bar{u}_{h}\right)=\frac{d}{d t}\left\|u_{h}\right\|^{2} .
$$

This property of our scheme is consistent with the energy conservation property of Schrödinger equations. It is essential to have a symmetric $A\left(u_{h}, v_{h}\right)$ for designing a finite element scheme which is energy-preserving for Schrödinger equations. Compared with discretization for diffusion equations, we don't have any extra diffusion term in (77) to help with the estimates. Therefore, superconvergence error estimates are more challenging compared with [6].

\section{Notations and Projections}

To facilitate the discussion, we introduce notations and define projection operators that will be used in the paper.

\subsection{Notations}

We introduce some notations first. We define the Legendre expansion of a function $u \in L^{2}(I)$ on cell $I_{j}$ as follows,

$$
\left.u\right|_{I_{j}}=\sum_{m=0}^{\infty} u_{j, m} L_{j, m}(x),
$$

where $L_{j, m}(x):=L_{m}(\xi), \xi=\frac{x-x_{j}}{h_{j} / 2}$, and $L_{m}(\cdot)$ is the standard Legendre polynomial of degree $m$ on $[-1,1]$. In what follows, we write $L_{j, m}(x)$ as $L_{j, m}$, and $L_{m}(\xi)$ as $L_{m}$ for notational convenience. We can compute $u_{j, m}$ using orthogonality of Legendre polynomials and Rodrigues' formula,

$$
\begin{aligned}
u_{j, m} & =\frac{2 m+1}{h_{j}} \int_{I_{j}} u(x) L_{j, m} d x=\frac{2 m+1}{2} \int_{-1}^{1} \hat{u}_{j}(\xi) L_{m} d \xi=\frac{2 m+1}{2} \frac{1}{2^{m} m !} \int_{-1}^{1} \hat{u}_{j}(\xi) \frac{d}{d \xi^{m}}\left(\xi^{2}-1\right)^{m} d \xi \\
& =\frac{2 m+1}{2} \frac{(-1)^{l}}{2^{m} m !} \int_{-1}^{1} \frac{d}{d \xi^{l}} \hat{u}_{j}(\xi) \frac{d}{d \xi^{m-l}}\left(\xi^{2}-1\right)^{m} d \xi
\end{aligned}
$$

where $\hat{u}_{j}(\xi)=u(x(\xi))$ is defined as the function $\left.u\right|_{I_{j}}$ transformed to the reference domain $[-1,1]$. By Holder's inequality, if $u \in W^{l, p}(I)$,

$$
\left|u_{j, m}\right| \leq C h_{j}^{l-\frac{1}{p}}|u|_{W^{l, p}\left(I_{j}\right)}, \quad 0 \leq l \leq m
$$

Similar to [8], we define operator $D^{-1}$ for any integrable function $v$ on $I_{j}$ by

$$
D^{-1} v(x)=\frac{2}{h_{j}} \int_{x_{j-\frac{1}{2}}}^{x} v(x) d x=\int_{-1}^{\xi} \hat{v}(\xi) d \xi, \quad x \in I_{j} .
$$


Table 1: Notations for some frequently used quantities. Subscript $j$ can be dropped for uniform mesh.

\begin{tabular}{|c|c|c|c|}
\hline Notation & Definition & Notation & Definition \\
\hline$G$ & {$\left[\begin{array}{cc}\frac{1}{2}+\alpha_{1} & -\beta_{2} \\
-\beta_{1} & \frac{1}{2}-\alpha_{1}\end{array}\right]$} & $H$ & {$\left[\begin{array}{cc}\frac{1}{2}-\alpha_{1} & \beta_{2} \\
\beta_{1} & \frac{1}{2}+\alpha_{1}\end{array}\right]$} \\
\hline$\Gamma_{j}$ & $\beta_{1}+\frac{\beta_{2}}{h_{j}^{2}} k^{2}\left(k^{2}-1\right)-\frac{2 k^{2}}{h_{j}}\left(\alpha_{1}^{2}\right.$ & $\Lambda_{j}$ & $-\frac{2 k}{h_{j}}\left(\alpha_{1}^{2}+\beta_{1} \beta_{2}-\frac{1}{4}\right)$ \\
\hline$L_{j, m}^{-}$ & $\begin{array}{c}L_{j, m}\left(x_{j+\frac{1}{2}}\right) \\
\frac{2}{h_{j}} \frac{d}{d x} L_{j, m}\left(x_{j+\frac{1}{2}}\right)\end{array}$ & $L_{j, m}^{+}$ & $\begin{array}{c}L_{j, m}\left(x_{j-\frac{1}{2}}\right) \\
\frac{2}{h_{j}} \frac{d}{d x} L_{j, m}\left(x_{j-\frac{1}{2}}\right)\end{array}$ \\
\hline$A_{j}$ & $G\left[L_{j, k-1}^{-}, L_{j, k}^{-}\right]$ & $B_{j}$ & $H\left[L_{j, k-1}^{+}, L_{j, k}^{+}\right]$ \\
\hline$r_{l}$ & $Q^{l}\left(I_{2}-Q^{N}\right)^{-1}$ & $\mathcal{M}_{j, m}$ & $\left(A_{j}+B_{j}\right)^{-1}\left(G L_{j, m}^{-}+H L_{j, m}^{+}\right)$ \\
\hline
\end{tabular}

Using the property of Legendre polynomials, we have

$$
\begin{aligned}
D^{-1} L_{j, k} & =\frac{1}{2 k+1}\left(L_{j, k+1}-L_{j, k-1}\right), \quad k \geq 1 \\
D^{-2} L_{j, k} & =\frac{1}{2 k+1}\left(\frac{1}{2 k+3}\left(L_{j, k+2}-L_{j, k}\right)-\frac{1}{2 k-1}\left(L_{j, k}-L_{j, k-2}\right)\right), \quad k \geq 2,
\end{aligned}
$$

where $D^{-2}=D^{-1} \circ D^{-1}$.

Finally, we collect some additional notations that will be frequently used in the paper in Table 1.

\subsection{Projections}

We summarize the definition and properties of projections in this subsection. We denote the standard $L^{2}$ projection of $u$ onto $V_{h}^{k}$ by $P_{h}^{0}$. Clearly, $\left.P_{h}^{0} u\right|_{I_{j}}=\sum_{m=0}^{k} u_{j, m} L_{j, m}$. The following projection $P_{h}^{\star}$ was introduced in [10].

Definition 3.1. For DG scheme with flux choice (5), we define the associated projection operator $P_{h}^{\star}$ for any periodic function $u \in W^{1, \infty}(I)$ to be the unique polynomial $P_{h}^{\star} u \in V_{h}^{k}$ (when $k \geq 1$ ) satisfying

$$
\begin{aligned}
\int_{I_{j}} P_{h}^{\star} u v_{h} d x & =\int_{I_{j}} u v_{h} d x & & \forall v_{h} \in P_{c}^{k-2}\left(I_{j}\right), \\
\widehat{P_{h}^{\star} u}=\left\{P_{h}^{\star} u\right\}-\alpha_{1}\left[P_{h}^{\star} u\right]+\beta_{2}\left[\left(P_{h}^{\star} u\right)_{x}\right] & =u & & \text { at } \quad x_{j+\frac{1}{2}}, \\
\widehat{\left(P_{h}^{\star} u\right)_{x}}=\left\{\left(P_{h}^{\star} u\right)_{x}\right\}+\alpha_{1}\left[\left(P_{h}^{\star} u\right)_{x}\right]+\beta_{1}\left[P_{h}^{\star} u\right] & =u_{x} & & \text { at } \quad x_{j+\frac{1}{2}},
\end{aligned}
$$

for all $j \in \mathbb{Z}_{N}$. When $k=1$, only conditions (13b)-(13c) are needed.

(13b)-(13c) is equivalent to

$$
\left.G\left[\begin{array}{c}
P_{h}^{\star} u \\
\left(P_{h}^{\star} u\right)_{x}
\end{array}\right]\right|_{x_{j+\frac{1}{2}}} ^{-}+\left.H\left[\begin{array}{c}
P_{h}^{\star} u \\
\left(P_{h}^{\star} u\right)_{x}
\end{array}\right]\right|_{x_{j+\frac{1}{2}}} ^{+}=\left.G\left[\begin{array}{c}
u \\
u_{x}
\end{array}\right]\right|_{x_{j+\frac{1}{2}}}+\left.H\left[\begin{array}{c}
u \\
u_{x}
\end{array}\right]\right|_{x_{j+\frac{1}{2}}} .
$$

In [10], the properties of $P_{h}^{\star}$ with general parameter choice $\alpha_{1}, \beta_{1}, \beta_{2}$ are shown, which is a key step to establish optimal convergence of the UWDG for many cases. In this paper, we only consider $k \geq 2$, which is required for superconvergence properties to hold. 
For completeness of the paper, we will briefly summarize the properties of $P_{h}^{\star}$ under the our assumptions as follows. Based on the results in [10], the existence and uniqueness of $P_{h}^{\star}$ is guaranteed if any of the following assumptions is satisfied.

- A1. (Local projection) scale invariant flux, $\alpha_{1}^{2}+\beta_{1} \beta_{2}=\frac{1}{4}$ and $\Gamma_{j} \neq 0$.

- A2. (Global projection) scale invariant flux, uniform mesh $\left(h_{j}=h, \forall j\right), \alpha_{1}^{2}+\beta_{1} \beta_{2} \neq \frac{1}{4}$ and $\left|\frac{\Gamma}{\Lambda}\right|>1$.

- A3. (Global projection) scale invariant flux, uniform mesh $\left(h_{j}=h, \forall j\right), \alpha_{1}^{2}+\beta_{1} \beta_{2} \neq \frac{1}{4}$. Besides, either $\left|\frac{\Gamma}{\Lambda}\right|=1,\left((-1)^{k+1} \frac{\Gamma}{\Lambda}\right)^{N} \neq 1$ with $N$ being an odd number or $\left|\frac{\Gamma}{\Lambda}\right|<1$, $\left((-1)^{k+1} \frac{\Gamma}{\Lambda}+\sqrt{\left(\frac{\Gamma}{\Lambda}\right)^{2}-1}\right)^{N} \neq 1$.

For example, alternating fluxes satisfy A1, central flux satisfies A2, IPDG, DDG and other more general fluxes may satisfy any of the assumptions A1/A2/A3 depending on the parameters. Here, the word "local" refers to the fact that $P_{h}^{\star}$ can be locally determined on each cell $I_{j}$. Otherwise $P_{h}^{\star}$ is a global projection and $P_{h}^{\star} u$ is the solution of a $2 N \times 2 N$ block-circulant system, where the coefficient matrix is

$$
M=\operatorname{circ}\left(A, B, 0_{2}, \cdots, 0_{2}\right),
$$

denoting a block-circulant matrix with first two rows as $\left(A, B, 0_{2}, \cdots, 0_{2}\right), 0_{2}$ is the $2 \times 2$ zero matrix, and $A, B$ are defined in Table 1 .

Assumptions A2 and A3 ensure the global matrix $M$ is invertible. It's known that

$$
M^{-1}=\operatorname{circ}\left(r_{0}, \cdots, r_{N-1}\right) \otimes A^{-1}
$$

where $\otimes$ means Kronecker product for block matrices,

$$
r_{j}=Q^{j}\left(I_{2}-Q^{N}\right)^{-1}, \quad Q=-A^{-1} B .
$$

With assumption A2, the eigenvalues of $Q$ are real and distinct. With assumption A3, the eigenvalues are either complex or repeated.

To shorten the notation, from here on we use two notations $C_{m}$ and $C_{m, n}$ to denote mesh independent constants. $C_{m}$ may depend on $|u|_{W^{k+1+m, \infty}(I)}$ for assumptions A1/A2, and $\|u\|_{W^{k+3+m, \infty}(I)}$ for assumption A3 when $\left|\frac{\Gamma}{\Lambda}\right|<1$, $\|u\|_{W^{k+4+m, \infty}(I)}$ for assumption A3 when $\left|\frac{\Gamma}{\Lambda}\right|=1$. $C_{m, n}$ may depend on $|u|_{W^{k+1+m+2 n, \infty(I)}}$ for assumptions A1/A2, on $\|u\|_{W^{k+3+m+3 n, \infty}(I)}$ for assumption A3 when $\frac{|\Gamma|}{|\Lambda|}<1$ and on $\|u\|_{W^{k+4+m+4 n, \infty}(I)}$ for assumption A3 when $\frac{|\Gamma|}{|\Lambda|}=1$.

We have the following lemma for some algebraic formulas that will be used several times in later sections.

Lemma 3.1. Suppose any of the assumptions A1/A2/A3 holds. Define

$$
\mathcal{M}_{j, m}=\left(A_{j}+B_{j}\right)^{-1}\left(G L_{j, m}^{-}+H L_{j, m}^{+}\right), \quad \forall m \in \mathbb{Z}^{+}, \forall j \in \mathbb{Z}_{N},
$$

where $G, H, A_{j}, B_{j}, L_{j, m}^{-}, L_{j, m}^{+}, \Gamma_{j}, \Lambda_{j}$ are defined in Table 1 . Then $\forall j \in \mathbb{Z}_{N}$,

$$
\left\|\left(A_{j}+B_{j}\right)^{-1} G\left[\begin{array}{cc}
1 & 0 \\
0 & \frac{1}{h_{j}}
\end{array}\right]\right\|_{\infty} \leq C,\left\|\left(A_{j}+B_{j}\right)^{-1} H\left[\begin{array}{cc}
1 & 0 \\
0 & \frac{1}{h_{j}}
\end{array}\right]\right\|_{\infty} \leq C,\left\|\mathcal{M}_{j, m}\right\|_{\infty} \leq C .
$$


Proof. If the mesh is uniform, the three matrices in (19) are independent of mesh size $h$ and the inequalities in (19) hold. For nonuniform mesh, the proof is given in Appendix A.2.

Since $P_{h}^{\star} u \in V_{h}^{k}$, it can be expressed in Legendre basis as

$$
\left.P_{h}^{\star} u\right|_{I_{j}}=\sum_{m=0}^{k} \dot{u}_{j, m} L_{j, m}
$$

By (13a), $u-P_{h}^{\star} u \perp V_{h}^{k-2}$, thus $u_{j, m}=u_{j, m}, \forall m \leq k-2$.

The following Lemma is a summary and extension of the results in Lemmas 3.2, 3.4, 3.8, 3.9 in [10].

Lemma 3.2. Suppose any of the assumptions A1/A2/A3 holds, for u satisfying the condition in Definition 3.1. If assumption $A 1$ is satisfied, then (14) is equivalent to $\forall j \in \mathbb{Z}_{N}$,

$$
\left.G\left[\begin{array}{c}
P_{h}^{\star} u \\
\left(P_{h}^{\star} u\right)_{x}
\end{array}\right]\right|_{x_{j+\frac{1}{2}}} ^{-}+\left.H\left[\begin{array}{c}
P_{h}^{\star} u \\
\left(P_{h}^{\star} u\right)_{x}
\end{array}\right]\right|_{x_{j-\frac{1}{2}}} ^{+}=\left.G\left[\begin{array}{c}
u \\
u_{x}
\end{array}\right]\right|_{x_{j+\frac{1}{2}}}+\left.H\left[\begin{array}{c}
u \\
u_{x}
\end{array}\right]\right|_{x_{j-\frac{1}{2}}},
$$

thus making $P_{h}^{\star}$ a local projection and

$$
\left[\begin{array}{c}
\dot{u}_{j, k-1} \\
\dot{u}_{j, k}
\end{array}\right]=\left[\begin{array}{c}
u_{j, k-1} \\
u_{j, k}
\end{array}\right]+\sum_{m=k+1}^{\infty} u_{j, m} \mathcal{M}_{j, m}
$$

If any of the assumptions A2/A3 is satisfied, then

$$
\left[\begin{array}{c}
\dot{u}_{j, k-1} \\
\dot{u}_{j, k}
\end{array}\right]=\left[\begin{array}{c}
u_{j, k-1} \\
u_{j, k}
\end{array}\right]+\sum_{m=k+1}^{\infty}\left(u_{j, m} V_{1, m}+\sum_{l=0}^{N-1} u_{j+l, m} r_{l} V_{2, m}\right)
$$

where $V_{1, m}=\left[L_{k-1}^{+}, L_{k}^{+}\right]^{-1} L_{m}^{+}, V_{2, m}=\left[L_{k-1}^{-}, L_{k}^{-}\right]^{-1} L_{m}^{-}-\left[L_{k-1}^{+}, L_{k}^{+}\right]^{-1} L_{m}^{+}$.

We have the following estimates

$$
\left|\dot{u}_{j, m}-u_{j, m}\right| \leq C_{0} h^{k+1}, m=k-1, k, \quad\left\|u-P_{h}^{\star} u\right\|_{L^{\nu}\left(\mathcal{I}_{N}\right)} \leq C_{0} h^{k+1}, \quad \nu=2, \infty .
$$

In addition, if $h_{j}=h_{j+1}$,

$$
\left|\dot{u}_{j, m}-u_{j, m}-\left(\dot{u}_{j+1, m}-u_{j+1, m}\right)\right| \leq C_{1} h^{k+2}, \quad m=k-1, k .
$$

Proof. Proof is given in Appendix A.3.

With the optimal estimates of $P_{h}^{\star} u$, we proved the optimal $L^{2}$ error estimate of the DG scheme in Theorem 3.10 in [10], which is restated below.

Theorem 3.3. [10] Suppose any of the assumptions A1/A2/A3 holds, let the exact solution $u$ of (1) be sufficiently smooth, satisfying periodic boundary condition and $u_{h}$ be the $D G$ solution in (41), then

$$
\left\|P_{h}^{\star} u-u_{h}\right\| \leq C_{2} h^{k+1}, \quad\left\|u-u_{h}\right\| \leq C_{2} h^{k+1} .
$$


Next, we introduce a local projection $P_{h}^{\dagger}$ as a variant of $P_{h}^{\star}$ and study its approximation properties. Similar ideas have been employed in [5] for proving the superconvergence at the so-called generalized Radau points when using upwind-biased flux for hyperbolic equations. This projection will help us reveal the superconvergence results at special points.

Definition 3.2. For DG scheme with flux choice (5), we define a local projection operator $P_{h}^{\dagger}$ associated with flux choice (5) for any periodic function $u \in W^{1, \infty}(I)$ to be the unique polynomial $P_{h}^{\dagger} u \in V_{h}^{k}$ (when $k \geq 1$ ) satisfying

$$
\begin{array}{r}
\int_{I_{j}} P_{h}^{\dagger} u v_{h} d x=\int_{I_{j}} u v_{h} d x, \quad \forall v_{h} \in P_{c}^{k-2}\left(I_{j}\right), \\
\left.G\left[\begin{array}{c}
P_{h}^{\dagger} u \\
\left(P_{h}^{\dagger} u\right)_{x}
\end{array}\right]\right|_{x_{j+\frac{1}{2}}} ^{-}+\left.H\left[\begin{array}{c}
P_{h}^{\dagger} u \\
\left(P_{h}^{\dagger} u\right)_{x}
\end{array}\right]\right|_{x_{j-\frac{1}{2}}} ^{+}=\left.G\left[\begin{array}{c}
u \\
u_{x}
\end{array}\right]\right|_{x_{j+\frac{1}{2}}}+\left.H\left[\begin{array}{c}
u \\
u_{x}
\end{array}\right]\right|_{x_{j-\frac{1}{2}}}
\end{array}
$$

for all $j \in \mathbb{Z}_{N}$. When $k=1$, only condition (27b) is needed.

Projection $P_{h}^{\dagger}$ is always a local projection. Denote $\left.P_{h}^{\dagger} u\right|_{I_{j}}=\sum_{m=0}^{k} \grave{u}_{j, m} L_{j, m}$, by (27a), $\grave{u}_{j, m}=u_{j, m}, m \leq k-2$. The similarities in definition imply that $P_{h}^{\star}$ and $P_{h}^{\dagger}$ are very close to each other, as shown in the following lemma.

Lemma 3.4. For periodic function $u \in W^{1, \infty}(I)$, if assumption $A 1$ is satisfied, $P_{h}^{\star} u=P_{h}^{\dagger} u$. If any of the assumptions A2/A3 is satisfied, $P_{h}^{\dagger}$ exists and is uniquely defined if $(-1)^{k+1} \frac{\Gamma_{j}}{\Lambda_{j}} \neq$ 1 for all $j \in \mathbb{Z}_{N}$. Then,

$$
\left\|u-P_{h}^{\dagger} u\right\|_{L^{\nu}\left(I_{j}\right)} \leq C h^{k+1}|u|_{W^{k+1, \nu}\left(I_{j}\right)}, \quad \nu=2, \infty .
$$

If any of the assumptions A2/A3 is satisfied, we have

$$
\left\|P_{h}^{\star} u-P_{h}^{\dagger} u\right\|_{L^{\nu}\left(\mathcal{I}_{\mathcal{N}}\right)} \leq C_{1} h^{k+2}, \quad \nu=2, \infty .
$$

Proof. When assumption A1 is satisfied, due to (21), $P_{h}^{\star}=P_{h}^{\dagger}$. The rest of the proof is given in Appendix A.4.

To analyze the superconvergence property at special points, we need to investigate the expansion of the projection error of $P_{h}^{\dagger}$ on every cell $I_{j}$

$$
\left.\left(u-P_{h}^{\dagger} u\right)\right|_{I_{j}}=\left[L_{j, k-1}, L_{j, k}\right]\left[\begin{array}{c}
u_{j, k-1}-\grave{u}_{j, k-1} \\
u_{j, k}-\grave{u}_{j, k}
\end{array}\right]+\sum_{m=k+1}^{\infty} u_{j, m} L_{j, m}=\sum_{m=k+1}^{\infty} u_{j, m} R_{j, m}, \quad \text { if } A 1 / A 2 / A 3,
$$

where $u_{j, m}$ is defined in (9) and

$$
R_{j, m}=L_{j, m}-\left[L_{j, k-1}, L_{j, k}\right] \mathcal{M}_{m}
$$

We write out the explicit expression of the leading term in expansions

$$
R_{j, k+1}=L_{j, k+1}+b L_{j, k}+c L_{j, k-1}
$$


where

$$
\begin{aligned}
& b=-\frac{2 \alpha_{1} \frac{2 k+1}{h_{j}}}{\Gamma_{j}+(-1)^{k} \Lambda_{j}}, \\
& c=-\frac{\beta_{1}-\frac{2(k+1)^{2}}{h_{j}}\left(\alpha_{1}^{2}+\beta_{1} \beta_{2}+\frac{1}{4}\right)-(-1)^{k+1} \frac{2(k+1)}{h_{j}}\left(\alpha_{1}^{2}+\beta_{1} \beta_{2}-\frac{1}{4}\right)+\frac{\beta_{2}}{h_{j}{ }^{2}} k(k+2)(k+1)^{2}}{\Gamma_{j}+(-1)^{k} \Lambda_{j}}
\end{aligned}
$$

to determine the location of superconvergent points.

For $s=0,1,2$, denote $D_{j}^{s}$ as the roots of $\frac{d^{s}}{d x^{s}} R_{j, k+1}, D^{s}=\bigcup_{j=1}^{N} D_{j}^{s}$, then it follows from (30) and (10) that, for $x \in D_{j}^{s}$,

$$
\partial^{s}\left(u-P_{h}^{\dagger} u\right)(x)=\sum_{m=k+2}^{\infty} u_{j, m} \frac{d^{s}}{d x^{s}} R_{j, m} \leq C h^{k+\frac{3}{2}-s}|u|_{W^{k+2, s}\left(I_{j}\right)}, \quad \text { if } A 1 / A 2 / A 3,
$$

indicating superconvergence at those points. For details, please see results in Theorem 4.6,

Since the expression of $b, c$ depends on $h_{j}$, on nonuniform mesh, $D_{j}^{s}, s=0,1,2$ have nodes with the different relative locations on each cell. For simplicity, below we discuss the locations of $D_{j}^{0}, D_{j}^{1}, D_{j}^{2}$ for special flux choices on uniform mesh.

- Alternating fluxes: $b= \pm \frac{2 k+1}{k}, c=-\frac{(k+1)^{2}}{k}$.

- Central flux: if $k$ is even, then $b=0, c=-\frac{(k+1)(k+2)}{k(k-1)}$; if $k$ is odd, then $b=0, c=-1$.

- IPDG fluxes: if $k$ is even, then $b=0, c=-\frac{(k+1)(k+2)-2 \tilde{\beta_{1}}}{k(k-1)-2 \tilde{\beta_{1}}}$; if $k$ is odd, then $b=0, c=-1$.

For central and IPDG fluxes, if $k$ is odd, $R_{j, k+1}=L_{j, k+1}-L_{j, k-1}, \frac{d}{d x} R_{j, k+1}=\frac{4 k+2}{h_{j}} L_{j, k}, \frac{d^{2}}{d x^{2}} R_{j, k+1}=$ $\frac{8 k+4}{h_{j}^{2}} L_{j, k}^{\prime}$, implying that $D_{j}^{0}, D_{j}^{1}, D_{j}^{2}$ are Lobatto points of order $k+1$, Gauss points of order $k$ and Lobatto points of order $k+1$ excluding end points, respectively, on interval $I_{j}$. Therefore, $\operatorname{card}\left(D_{j}^{0}\right)=k+1, \operatorname{card}\left(D_{j}^{1}\right)=k, \operatorname{card}\left(D_{j}^{2}\right)=k-1$.

Cao et al. proved there exists $k+1$ superconvergence points (Radau points) when using upwind flux for linear hyperbolic problem in [8], $k+1$ superconvergence points (Lobatto points) using special flux parameter in DDG method in [6] and $k+1$ or $k$ superconvergence points, depending on parameters, for using upwind-biased flux for linear hyperbolic problem in [5]. Analyzing the number and location of superconvergent points for our scheme is more challenging. We shall only provide lower bound estimates for the number of superconvergence points. For general parameters choices, when $k \geq 2, R_{j, k+1} \perp P_{c}^{k-2}\left(I_{j}\right)$, by Theorem 3.3 and Corollary 3.4 in [23], we can easily show $R_{j, k+1}$ has at least $k-1$ simple zeros, i.e., $\operatorname{card}\left(D_{j}^{0}\right) \geq k-1$. By the same approach, we can show when $k \geq 3, \operatorname{card}\left(D_{j}^{1}\right) \geq k-2$, and when $k \geq 4, \operatorname{card}\left(D_{j}^{2}\right) \geq k-3$. For small $k$ values, $D_{j}^{1}, D_{j}^{2}$ can possibly be empty sets.

\section{Superconvergence Properties}

In this section, we study superconvergence of the numerical solution. First, we investigate the superconvergence of UWDG fluxes, cell averages, towards a particular projection and at some special points. This analysis is done by decomposing the error into

$$
e=u-u_{h}=\epsilon_{h}+\zeta_{h}, \epsilon_{h}=u-u_{I}, \zeta_{h}=u_{I}-u_{h}
$$


for some $u_{I} \in V_{h}^{k}$. For error analysis of DG schemes, $u_{I}$ is usually taken as some projection of $u$. While for our purpose of superconvergence analysis, $u_{I}$ needs to be carefully designed as illustrated in Section 4.2. First, without specifying $u_{I}$, we prove some intermediate superconvergence results in Section 4.1. Then, the choice of $u_{I}$ is made in Section 4.2 and the main results are obtained. The other superconvergence property is about the negative norm of the UWDG solution, which enables a post-processing technique to obtain highly accurate solutions. This is considered in Section 4.3.

\subsection{Some intermediate superconvergence results}

This subsection will collect superconvergence results of $\left\|\left(\zeta_{h}\right)_{x x}\right\|_{L^{2}(I)},\left(\frac{1}{N} \sum_{j=1}^{N}\left|\left[\zeta_{h}\right]\right|_{j+\frac{1}{2}}^{2}\right)^{\frac{1}{2}}$, $\left(\frac{1}{N} \sum_{j=1}^{N}\left|\left[\left(\zeta_{h}\right)_{x}\right]\right|_{j+\frac{1}{2}}^{2}\right)^{\frac{1}{2}}$ without specifying $u_{I}$. The main idea is to choose special test functions in error equation, similar to the techniques used in [12] for hyperbolic problems. This is an essential step to elevate the superconvergence order in Theorem 4.4 when $k$ is even.

Lemma 4.1. For $k \geq 2$, let $u$ be the exact solution to (1) and $u_{h}$ be the $D G$ solution in (44). $\epsilon_{h}, \zeta_{h}$ are defined in (34). We define $s_{h}$ to be a unique function in $V_{h}^{k}$, such that $\int_{I} s_{h} v_{h} d x=a\left(\epsilon_{h}, v_{h}\right), \forall v_{h} \in V_{h}^{k}$. Then, when any of the assumptions A1/A2 is satisfied, we have

$$
\begin{aligned}
\left\|\left(\zeta_{h}\right)_{x x}\right\| & \leq C\left\|s_{h}+\left(\zeta_{h}\right)_{t}\right\|, \\
\left(\frac{1}{N} \sum_{j=1}^{N}\left|\left[\zeta_{h}\right]\right|_{j+\frac{1}{2}}^{2}\right)^{\frac{1}{2}} & \leq C h^{2}\left\|s_{h}+\left(\zeta_{h}\right)_{t}\right\|, \\
\left(\frac{1}{N} \sum_{j=1}^{N}\left|\left[\left(\zeta_{h}\right)_{x}\right]\right|_{j+\frac{1}{2}}^{2}\right)^{\frac{1}{2}} & \leq C h\left\|s_{h}+\left(\zeta_{h}\right)_{t}\right\| .
\end{aligned}
$$

Proof. The proof is given in Appendix A.5.

\subsection{Correction functions and the main results}

In this section, we shall present the main superconvergence results. The proof depends on Lemma 4.1 and the correction function technique introduced by Cao et al. in [8, 6], which is essential for superconvergence. We let $u_{I}=P_{h}^{\star} u$ when $k=2$, and $u_{I}=P_{h}^{\star} u-w$, when $k \geq 3$, where $w \in V_{h}^{k}$ is a specially designed correction function defined below.

Similar to [6], we start the construction by defining $w_{q}, 1 \leq q \leq\left\lfloor\frac{k-1}{2}\right\rfloor$. For $k \geq 3$, we denote $w_{0}=u-P_{h}^{\star} u$ and define a series of functions $w_{q} \in V_{h}^{k}$, as follows

$$
\begin{aligned}
\int_{I_{j}} w_{q}\left(v_{h}\right)_{x x} d x & =-i \int_{I_{j}}\left(w_{q-1}\right)_{t} v_{h} d x, & & \forall v_{h} \in P_{c}^{k}\left(I_{j}\right) \backslash P_{c}^{1}\left(I_{j}\right), \\
\widehat{w_{q}} & =0, & & \text { at } x_{j+\frac{1}{2}}, \\
\widetilde{\left(w_{q}\right)_{x}} & =0, & & \text { at } x_{j+\frac{1}{2}},
\end{aligned}
$$


for all $j \in \mathbb{Z}_{N} \cdot(\underline{38 \mathrm{~b}})$ and $(\underline{38 \mathrm{c}})$ is equivalent to

$$
\left.G\left[\begin{array}{c}
w_{q} \\
\left(w_{q}\right)_{x}
\end{array}\right]\right|_{x_{j+\frac{1}{2}}} ^{-}+\left.H\left[\begin{array}{c}
w_{q} \\
\left(w_{q}\right)_{x}
\end{array}\right]\right|_{x_{j+\frac{1}{2}}} ^{+}=0 .
$$

$w_{q}$ exists and is unique when any of the assumptions A1/A2/A3 is satisfied for the same reason as the existence and uniqueness of $P_{h}^{\star}$.

With the construction of $w_{q}$, we define

$$
w(x, t)=\sum_{q=1}^{\left\lfloor\frac{k-1}{2}\right\rfloor} w_{q}(x, t),
$$

then

$$
\begin{aligned}
a_{j}\left(\epsilon_{h}, v_{h}\right) & =a_{j}\left(u-P_{h}^{\star} u, v_{h}\right)+\sum_{q=1}^{\left\lfloor\frac{k-1}{2}\right\rfloor} a_{j}\left(w_{q}, v_{h}\right) \\
& =\int_{I_{j}}\left(w_{0}\right)_{t} v_{h} d x+\sum_{q=1}^{\left\lfloor\frac{k-1}{2}\right\rfloor}\left(\int_{I_{j}}\left(w_{q}\right)_{t} v_{h} d x-i \int_{I_{j}} w_{q}\left(v_{h}\right)_{x x} d x\right) \\
& =\int_{I_{j}}\left(w_{0}\right)_{t} v_{h} d x+\sum_{q=1}^{\left\lfloor\frac{k-1}{2}\right\rfloor} \int_{I_{j}}\left(w_{q}-w_{q-1}\right)_{t} v_{h} d x \\
& =\int_{I_{j}}\left(w_{\left\lfloor\frac{k-1}{2}\right\rfloor}\right)_{t} v_{h} d x, \quad \forall v_{h} \in V_{h}^{k}(I) .
\end{aligned}
$$

The approximation property of $w_{q}$ and $a_{j}\left(\epsilon_{h}, v_{h}\right)$ are presented in the following Lemma.

Lemma 4.2. For $k \geq 3$, suppose $u$ satisfies the condition in Theorem 3.3. For $w_{q}, 1 \leq q \leq$ $\left\lfloor\frac{k-1}{2}\right\rfloor, q+r \leq\left\lfloor\frac{k-1}{2}\right\rfloor+1$, we have

$$
\begin{array}{r}
\left.\partial_{t}^{r} w_{q}\right|_{I_{j}}=\sum_{m=k-1-2 q}^{k} \partial_{t}^{r} c_{j, m}^{q} L_{j, m}, \quad \partial_{t}^{r} c_{j, k-1-2 q}^{q}=C h_{j}^{2 q} \partial_{t}^{q+r}\left(u_{j, k-1}-\dot{u}_{j, k-1}\right), \\
\left|\partial_{t}^{r} c_{j, m}^{q}\right| \leq C_{2 r, q} h^{k+1+2 q},
\end{array}
$$

and then

$$
\left\|\partial_{t}^{r} w_{q}\right\| \leq C_{2 r, q} h^{k+1+2 q} .
$$

For any $v_{h} \in V_{h}^{k}$,

$$
\left|a\left(\epsilon_{h}, v_{h}\right)\right| \leq C_{2,\left\lfloor\frac{k-1}{2}\right\rfloor} h^{k+1+2\left\lfloor\frac{k-1}{2}\right\rfloor}\left\|v_{h}\right\| .
$$

Proof. The proof is given in Appendix A.6. 
Lemma 4.3. For $k \geq 2$, suppose $u$ satisfies the condition in Theorem 3.3. If the parameters satisfy any of the assumptions $A 1 / A 2$ and $\left.u_{h}\right|_{t=0}=\left.u_{I}\right|_{t=0}$, we have

$$
\begin{aligned}
&\left\|\left(\zeta_{h}\right)_{x x}\right\| \leq C_{4+2\left\lfloor\frac{k-1}{2}\right\rfloor} h^{k+1+2\left\lfloor\frac{k-1}{2}\right\rfloor} . \\
&\left(\frac{1}{N} \sum_{j=1}^{N}\left|\left[\left(\zeta_{h}\right)\right]\right|_{j+\frac{1}{2}}^{2}\right)^{\frac{1}{2}} \leq C_{4+2\left\lfloor\frac{k-1}{2}\right\rfloor} h^{k+3+2\left\lfloor\frac{k-1}{2}\right\rfloor} . \\
&\left(\frac{1}{N} \sum_{j=1}^{N}\left|\left[\left(\zeta_{h}\right)_{x}\right]\right|_{j+\frac{1}{2}}^{2}\right)^{\frac{1}{2}} \leq C_{4+2\left\lfloor\frac{k-1}{2}\right\rfloor} h^{k+2+2\left\lfloor\frac{k-1}{2}\right\rfloor} .
\end{aligned}
$$

Proof. When $k=2, w=0 . a\left(\epsilon_{h}, v_{h}\right)=\int_{I}\left(\epsilon_{h}\right)_{t} v_{h} d x$ from the definition of $P_{h}^{\star}$. That is, $s_{h}=\left(\epsilon_{h}\right)_{t}$ in the condition of Lemma 4.1. To bound $\left\|\left(\zeta_{h}\right)_{t}\right\|$, we take the time derivative of the error equation and obtain

$$
a\left(e_{t}, v_{h}\right)=a\left(\left(\epsilon_{h}\right)_{t}, v_{h}\right)+a\left(\left(\zeta_{h}\right)_{t}, v_{h}\right)=0 .
$$

Let $v_{h}=\overline{\left(\zeta_{h}\right)_{t}}$, by (7) and the property of $P_{h}^{\star} u$, we obtain

$$
\frac{d}{d t}\left\|\left(\zeta_{h}\right)_{t}\right\|^{2}=-a\left(\left(\epsilon_{h}\right)_{t}, \overline{\left(\zeta_{h}\right)_{t}}\right)-\overline{a\left(\left(\epsilon_{h}\right)_{t}, \overline{\left(\zeta_{h}\right)_{t}}\right)} \leq 2\left\|\left(\epsilon_{h}\right)_{t t}\right\|\left\|\left(\zeta_{h}\right)_{t}\right\|
$$

which implies $\frac{d}{d t}\left\|\left(\zeta_{h}\right)_{t}\right\| \leq\left\|\left(\epsilon_{h}\right)_{t t}\right\|$. To estimate $\left\|\left.\left(\zeta_{h}\right)_{t}\right|_{t=0}\right\|$, we let $t=0$ in the error equation. Since $\left.\zeta_{h}\right|_{t=0}=\left.\left(u_{h}-u_{I}\right)\right|_{t=0}=0$, we have

$$
a\left(\epsilon_{h}, v_{h}\right)+\left.\int_{I}\left(\zeta_{h}\right)_{t}\right|_{t=0} v_{h} d x=0
$$

Let $v_{h}=\left.\overline{\left(\zeta_{h}\right)_{t}}\right|_{t=0}$, then

$$
\left\|\left.\left(\zeta_{h}\right)_{t}\right|_{t=0}\right\|^{2} \leq\left\|\left(\epsilon_{h}\right)_{t}\right\|\left\|\left.\left(\zeta_{h}\right)_{t}\right|_{t=0}\right\|
$$

Therefore,

$$
\left\|\left(\zeta_{h}\right)_{t}\right\| \leq\left\|\left(\epsilon_{h}\right)_{t}\right\|+t\left\|\left(\epsilon_{h}\right)_{t t}\right\| .
$$

By Lemma 4.1, estimates in (24) and the inequality above, we can get (45)-(46).

For $k \geq 3$, by (41), we have $a\left(\epsilon_{h}, v_{h}\right)=\int_{I}\left(w_{\left\lfloor\frac{k-1}{2}\right\rfloor}\right)_{t} v_{h} d x$, that is, $s_{h}=\left(w_{\left\lfloor\frac{k-1}{2}\right\rfloor}\right)_{t}$ in the condition of Lemma 4.1. Then, following the same lines of proof as above, by replacing $\epsilon_{h}$ with $w_{\left\lfloor\frac{k-1}{2}\right\rfloor}$ and using Lemma 4.2, we are done.

Now we are ready to state the following estimates of $\left\|\zeta_{h}\right\|$.

Theorem 4.4. For $k \geq 2$, suppose $u$ satisfies the condition in Theorem 3.3 . Assume $\left.u_{h}\right|_{t=0}=$ $\left.u_{I}\right|_{t=0}$, then $\forall t \in\left(0, T_{e}\right]$,

$$
\left\|\zeta_{h}\right\| \leq \begin{cases}C_{2, \frac{k-1}{2}} h^{2 k} & \text { if } k \text { is odd and } A 1 / A 2 / A 3, \\ \left(C_{k+2} h^{4 k}+\sum_{I_{j} \subset I^{N U}} C_{k} h^{4 k-1}\right)^{\frac{1}{2}} & \text { if } k \text { is even and A1/A2, } \\ C_{2, \frac{k-2}{2}} h^{2 k-1}, & \text { if } k \text { is even and } A 3,\end{cases}
$$

where $I^{N U}$ is the collection of cells in which the length of $I_{j}$ is different with at least one of its neighbors. 
Proof. From error equation, $a\left(e, \overline{\zeta_{h}}\right)=a\left(\epsilon_{h}, \overline{\zeta_{h}}\right)+a\left(\zeta_{h}, \overline{\zeta_{h}}\right)=0$, which gives us

$$
\frac{d}{d t}\left\|\zeta_{h}\right\|^{2}=-a\left(\epsilon_{h}, \overline{\zeta_{h}}\right)-\overline{a\left(\epsilon_{h}, \overline{\zeta_{h}}\right)} \leq \begin{cases}2\left\|\left(\epsilon_{h}\right)_{t}\right\|\left\|\zeta_{h}\right\|, & k=2 \\ 2\left\|\left(w_{\left\lfloor\frac{k-1}{2}\right\rfloor}\right)_{t}\right\|\left\|\zeta_{h}\right\|, & k \geq 3\end{cases}
$$

By (24), (43) and Gronwall's inequality, we have

$$
\left\|\zeta_{h}\right\| \leq C_{2,\left\lfloor\frac{k-1}{2}\right\rfloor} t h^{k+1+2\left\lfloor\frac{k-1}{2}\right\rfloor}, \quad \forall t \in\left(0, T_{e}\right] .
$$

Therefore, when $k$ is odd , or $k$ is even and parameters satisfy A3, the proof is complete.

When $k$ is even and parameters satisfy any of the assumptions A1/A2, we make use of Lemma 4.3 to show the improved estimates. We let $l=\left\lfloor\frac{k-1}{2}\right\rfloor=\frac{k-2}{2}$, then

$$
\begin{aligned}
a\left(\epsilon_{h}, \overline{\zeta_{h}}\right) & =\int_{I}\left(w_{l}\right)_{t} \overline{\zeta_{h}} d x=\sum_{j=1}^{N} \sum_{m=1}^{k} \partial_{t} c_{j, m}^{l} \int_{I_{j}} L_{j, m} \overline{\zeta_{h}} d x \\
& =\sum_{j=1}^{N} \partial_{t} c_{j, 1}^{l} \int_{I_{j}} L_{j, 1} \overline{\zeta_{h}} d x+\sum_{j=1}^{N} \sum_{m=2}^{k} \partial_{t} c_{j, m}^{l} \int_{I_{j}} L_{j, m} \overline{\zeta_{h}} d x \doteq \mathcal{A}_{1}+\mathcal{A}_{2},
\end{aligned}
$$

where we denote the first term in the summation by $\mathcal{A}_{1}$, and the other summation term as $\mathcal{A}_{2}$. Note that $D^{-1} L_{j, m} \perp P^{0}, m \geq 1$ in the inner product sense, thus $D^{-2} L_{j, m}( \pm 1)=0, m \geq 2$. By integration by parts, we get

$$
\begin{array}{rl}
\mathcal{A}_{2} & =\sum_{j=1}^{N} \sum_{m=2}^{k} \frac{h_{j}^{2}}{4} \partial_{t} c_{j, m}^{l} \int_{I_{j}} D^{-2} L_{j, m} \overline{\left(\zeta_{h}\right)} \\
x x & d x \\
& \leq C h^{-1} \sum_{j=1}^{N}\left|\frac{h_{j}^{2}}{4} \partial_{t} c_{j, m}^{l}\right|^{2}+h \sum_{j=1}^{N} \sum_{m=2}^{k}\left(\int_{I_{j}} D^{-2} L_{j, m} \overline{\left(\zeta_{h}\right)}\right. \\
x x & d x)^{2} \leq C_{k} h^{4 k}+C h^{2}\left\|\left(\zeta_{h}\right)_{x x}\right\|^{2} \leq C_{k+2} h^{4 k}
\end{array}
$$

where we have used (42) in the first inequality, and (45) in the third inequality.

To estimate $\mathcal{A}_{1}$, we take the first and second antiderivative of $L_{j, 1}=\xi$ as $\frac{h_{j}}{2}\left(\frac{\xi^{2}}{2}-\frac{1}{6}\right)$, $\left(\frac{h_{j}}{2}\right)^{2} \frac{\xi^{3}-\xi}{6}$ and apply integration by parts twice,

$$
\begin{aligned}
\mathcal{A}_{1} & =\sum_{j=1}^{N} \frac{h_{j}}{2} \partial_{t} c_{j, 1}^{l}\left(\left.\left(\frac{\xi^{2}}{2}-\frac{1}{6}\right) \bar{\zeta}_{h}\right|_{x_{j-\frac{1}{2}}} ^{x_{j+\frac{1}{2}}}-\int_{I_{j}}\left(\frac{\xi^{2}}{2}-\frac{1}{6}\right)\left(\bar{\zeta}_{h}\right)_{x} d x\right) \\
& =\sum_{j=1}^{N} \frac{h_{j}}{2} \partial_{t} c_{j, 1}^{l}\left(\frac{1}{3}\left(\left.\bar{\zeta}_{h}\right|_{j+\frac{1}{2}} ^{-}-\left.\bar{\zeta}_{h}\right|_{j-\frac{1}{2}} ^{+}\right)+\frac{h_{j}}{2} \int_{I_{j}} \frac{\xi^{3}-\xi}{6}\left(\bar{\zeta}_{h}\right)_{x x} d x\right) \\
& =\sum_{j=1}^{N} \frac{h_{j}}{2} \partial_{t} c_{j, 1}^{l}\left(-\frac{1}{3}\left[\bar{\zeta}_{h}\right]_{j+\frac{1}{2}}+\frac{h_{j}}{2} \int_{I_{j}} \frac{\xi^{3}-\xi}{6}\left(\bar{\zeta}_{h}\right)_{x x} d x\right) \\
& +\left.\sum_{j=1}^{N}\left(\left(\frac{h_{j}}{2}\right) \partial_{t} c_{j, 1}^{l}-\left(\frac{h_{j+1}}{2}\right) \partial_{t} c_{j+1,1}^{l}\right) \frac{1}{3} \bar{\zeta}_{h}\right|_{j+\frac{1}{2}} ^{+},
\end{aligned}
$$


where we have used the periodicity in the last equality. Therefore,

$$
\begin{aligned}
\left|\mathcal{A}_{1}\right| & \leq \frac{1}{2} h \sum_{j=1}^{N}\left(\left(\frac{h_{j}}{2}\right)\left|\partial_{t} c_{j, 1}^{l}\right|\right)^{2}+h^{-1} \sum_{j=1}^{N} \frac{1}{9}\left|\left[\bar{\zeta}_{h}\right]\right|_{j+\frac{1}{2}}^{2}+C h^{2} \sum_{j=1}^{N}\left\|\left(\zeta_{h}\right)_{x x}\right\|_{L^{2}\left(I_{j}\right)}^{2} \\
& +\frac{1}{18} h^{-1} \sum_{j=1}^{N}\left|\left(\left(\frac{h_{j}}{2}\right) \partial_{t} c_{j, 1}^{l}-\left(\frac{h_{j+1}}{2}\right) \partial_{t} c_{j+1,1}^{l}\right)\right|^{2}+h \sum_{j=1}^{N}\left\|\zeta_{h}\right\|_{L^{2}\left(\partial I_{j}\right)}^{2} \\
& \leq C_{k+2} h^{4 k}+C\left\|\zeta_{h}\right\|^{2}+C h^{-1} \sum_{j=1}^{N}\left|\left(\left(\frac{h_{j}}{2}\right) \partial_{t} c_{j, 1}^{l}-\left(\frac{h_{j+1}}{2}\right) \partial_{t} c_{j+1,1}^{l}\right)\right|^{2}
\end{aligned}
$$

where we used (42), inverse inequality, (45) and (46) in the last inequality.

We estimate the last term in $\mathcal{A}_{1}$ by the estimation of the difference of $u_{j, m}$ in neighboring cells, similar to that in Proposition 3.1 of [3]. If $h_{j} \neq h_{j+1}$, then

$$
\left(\frac{h_{j}}{2}\right) \partial_{t} c_{j, 1}^{l}-\left(\frac{h_{j+1}}{2}\right) \partial_{t} c_{j+1,1}^{l} \leq C_{k} h^{2 k}
$$

If $h_{j}=h_{j+1}$, by (42) and (25),

$\left(\frac{h_{j}}{2}\right) \partial_{t} c_{j, 1}^{l}-\left(\frac{h_{j+1}}{2}\right) \partial_{t} c_{j+1,1}^{l}=C\left(\frac{h_{j}}{2}\right)^{2 l+1} \partial_{t}^{l+1}\left(u_{j, k-1}-\dot{u}_{j, k-1}-\left(u_{j+1, k-1}-\dot{u}_{j+1, k-1}\right)\right) \leq C_{k+1} h^{2 k+1}$.

Therefore, we have

$$
\left|\mathcal{A}_{1}\right| \leq C_{k+2} h^{4 k}+C\left\|\zeta_{h}\right\|^{2}+\sum_{I_{j} \subset I^{N U}} C_{k} h^{4 k-1}
$$

Combine with the estimates for $\mathcal{A}_{2}$, we have

$$
\frac{d}{d t}\left\|\zeta_{h}\right\|^{2} \leq C_{k+2} h^{4 k}+C\left\|\zeta_{h}\right\|^{2}+\sum_{I_{j} \subset I^{N U}} C_{k} h^{4 k-1}
$$

By Gronwall's inequality and the numerical initial condition, we obtain

$$
\left\|\zeta_{h}\right\| \leq\left(C_{k+2} h^{4 k}+\sum_{I_{j} \subset I^{N U}} C_{k} h^{4 k-1}\right)^{\frac{1}{2}}
$$

The proof is now complete.

With Theorem 4.4, we are able to prove the following superconvergence results.

Theorem 4.5 (Superconvergence of numerical fluxes and cell averages). Let

$E_{f}=\left(\left.\frac{1}{N} \sum_{j=1}^{N}\left(u-\widehat{u_{h}}\right)\right|_{j+\frac{1}{2}} ^{2}\right)^{\frac{1}{2}}, \quad E_{f_{x}}=\left(\left.\frac{1}{N} \sum_{j=1}^{N}\left(u_{x}-\widetilde{\left(u_{h}\right)_{x}}\right)\right|_{j+\frac{1}{2}} ^{2}\right)^{\frac{1}{2}}, \quad E_{c}=\left(\frac{1}{N} \sum_{j=1}^{N}\left|\frac{1}{h_{j}} \int_{I_{j}} u-u_{h} d x\right|^{2}\right)^{\frac{1}{2}}$

be the errors in the two numerical fluxes and the cell averages, respectively. For $k \geq 2$, suppose $u$ satisfies the condition in Theorem [3.3. Assume $\left.u_{h}\right|_{t=0}=\left.u_{I}\right|_{t=0}$, then $\forall t \in\left(0, T_{e}\right]$ 
- if $k$ is odd, parameters satisfy any of the assumptions A1/A2/A3, we have

$$
E_{f} \leq C_{2, \frac{k-1}{2}} h^{2 k}, \quad E_{f_{x}} \leq C_{2, \frac{k-1}{2}} h^{2 k-1}, \quad E_{c} \leq C_{2, \frac{k-1}{2}} h^{2 k},
$$

- if $k$ is even, parameters satisfy any of the assumptions A1/A2, we have

$$
\begin{aligned}
& E_{f} \leq\left(C_{k+2} h^{4 k}+\sum_{I_{j} \subset I^{N U}} C_{k} h^{4 k-1}\right)^{\frac{1}{2}}, E_{f_{x}} \leq\left(C_{k+2} h^{4 k}+\sum_{I_{j} \subset I^{N U}} C_{k} h^{4 k-1}\right)^{\frac{1}{2}} h^{-1}, \\
& E_{c} \leq\left(C_{k+2} h^{4 k}+\sum_{I_{j} \subset I^{N U}} C_{k} h^{4 k-1}\right)^{\frac{1}{2}},
\end{aligned}
$$

where $I^{N U}$ is the collection of cells in which the length of $I_{j}$ is different with at least one of its neighbors.

- if $k$ is even and parameters satisfy assumption A3, we have

$$
E_{f} \leq C_{2, \frac{k-2}{2}} h^{2 k-1}, \quad E_{f_{x}} \leq C_{2, \frac{k-2}{2}} h^{2 k-2}, \quad E_{c} \leq C_{2, \frac{k-2}{2}} h^{2 k-1} .
$$

Proof. We first prove the estimates for $E_{f}$. By (38b) and the definition of $P_{h}^{\star}, \widehat{\epsilon}_{h}\left(x_{j+\frac{1}{2}}\right)=$ $\widehat{u-u_{I}}\left(x_{j+\frac{1}{2}}\right)=0$, then

$$
\left.\left(u-\widehat{u_{h}}\right)\right|_{j+\frac{1}{2}}=\left.\left(\widehat{\zeta_{h}}\right)\right|_{j+\frac{1}{2}}=\left.\left(\left\{\zeta_{h}\right\}-\alpha_{1}\left[\zeta_{h}\right]+\beta_{2}\left[\left(\zeta_{h}\right)_{x}\right]\right)\right|_{j+\frac{1}{2}} .
$$

Therefore, by inverse inequality and the fact $\beta_{2}=\tilde{\beta}_{2} h$,

$$
\left(E_{f}\right)^{2} \leq\left(\frac{1}{N}\left\|\zeta_{h}\right\|_{L^{2}\left(\partial \mathcal{I}_{N}\right)}^{2}\right)^{\frac{1}{2}} \leq C\left\|\zeta_{h}\right\|,
$$

and the desired estimates for $E_{f}$ is obtained by (48). The estimates for $E_{f_{x}}$ can be obtained following same lines.

Next, we prove the estimates for $E_{c}$. If $k$ is odd, then $\int_{I_{j}} w_{q} d x=0,1 \leq q \leq \frac{k-3}{2}$, by (42) and orthogonality of Legendre polynomials. Thus,

$$
\int_{I_{j}} u-u_{h} d x=\int_{I_{j}} u-P_{h}^{\star} u+\sum_{q=1}^{\left\lfloor\frac{k-1}{2}\right\rfloor} w_{q}+\zeta_{h} d x=\int_{I_{j}} w_{\left\lfloor\frac{k-1}{2}\right\rfloor} d x+\int_{I_{j}} \zeta_{h} d x .
$$

By the Cauchy-Schwartz inequality, we have

$$
\left|\frac{1}{h_{j}} \int_{I_{j}} u-u_{h} d x\right|^{2} \leq \frac{1}{h_{j}}\left(\left\|\zeta_{h}\right\|_{L^{2}\left(I_{j}\right)}^{2}+\left\|w_{\frac{k-1}{2}}\right\|_{L^{2}\left(I_{j}\right)}^{2}\right) .
$$

If $k$ is even, then $\int_{I_{j}} w_{q} d x=0,1 \leq q \leq \frac{k-2}{2}$, by (42) and orthogonality of Legendre polynomials. Thus, by similar step, we have

$$
\int_{I_{j}} u-u_{h} d x=\int_{I_{j}} \zeta_{h} d x, \quad\left|\frac{1}{h_{j}} \int_{I_{j}} u-u_{h} d x\right|^{2} \leq \frac{1}{h_{j}}\left\|\zeta_{h}\right\|_{L^{2}\left(I_{j}\right)}^{2} .
$$

Therefore,

$$
E_{c} \leq C\left(\left\|\zeta_{h}\right\|^{2}+\left\|w_{\frac{k-1}{2}}\right\|^{2}\right)^{1 / 2} \text { if } k \text { is odd, } E_{c} \leq C\left(\left\|\zeta_{h}\right\|^{2}\right)^{1 / 2} \text { if } k \text { is even, }
$$

and the desired estimate for $E_{c}$ is obtained by (48) and (43). 
Theorem 4.6 (Superconvergence towards projections and at special points). Suppose $u$ satisfies the condition in Theorem 3.3. Assume $\left.u_{h}\right|_{t=0}=P_{h}^{\star} u_{0}$, then $\forall t \in\left(0, T_{e}\right]$,

$$
\left\|u_{h}-P_{h}^{\star} u\right\| \leq \begin{cases}\left(C_{4} h^{4 k}+\sum_{I_{j} \subset I^{N U}} C_{2} h^{4 k-1}\right)^{\frac{1}{2}} & k=2, \text { if } A 1 \text { or A2 } \\ C_{2,1}(1+t) h^{k+3} & k \geq 3,\end{cases}
$$

where $I^{N U}$ is the collection of cells in which the length of $I_{j}$ is different with at least one of its neighbors.

If $D^{s}, s=0,1,2$ defined in (33) are not empty sets. Let

$$
\begin{aligned}
& E_{u}=\left(\frac{1}{\left|D^{0}\right|} \sum_{x \in D^{0}}\left|\left(u-u_{h}\right)(x)\right|^{2}\right)^{\frac{1}{2}}, \quad E_{u_{x}}=\left(\frac{1}{\left|D^{1}\right|} \sum_{x \in D^{1}}\left|\left(u-u_{h}\right)_{x}(x)\right|^{2}\right)^{\frac{1}{2}}, \\
& E_{u_{x x}}=\left(\frac{1}{\left|D^{2}\right|} \sum_{x \in D^{2}}\left|\left(u-u_{h}\right)_{x x}(x)\right|^{2}\right)^{\frac{1}{2}}
\end{aligned}
$$

be the average point value error for the numerical solution, the derivative of solution and the second order derivative of solution at corresponding sets of points. Then

- if $k=2$ and any of the assumptions A1/A2 is satisfied, we have

$$
\begin{aligned}
& E_{u} \leq\left(C_{4} h^{4 k}+\sum_{I_{j} \subset I^{N U}} C_{2} h^{4 k-1}\right)^{\frac{1}{2}}, \quad E_{u_{x}} \leq h^{-1}\left(C_{4} h^{4 k}+\sum_{I_{j} \subset I^{N U}} C_{2} h^{4 k-1}\right)^{\frac{1}{2}}, \\
& E_{u_{x x}} \leq h^{-2}\left(C_{4} h^{4 k}+\sum_{I_{j} \subset I^{N U}} C_{2} h^{4 k-1}\right)^{\frac{1}{2}} .
\end{aligned}
$$

- if $k \geq 3$ and any of the assumptions A1/A2/A3 is satisfied, we have

$$
E_{u} \leq C_{2,1} h^{k+2}, \quad E_{u_{x}} \leq C_{2,1} h^{k+1}, \quad E_{u_{x x}} \leq C_{2,1} h^{k} .
$$

Proof. When $k=2$, we have $u_{h}-P_{h}^{\star} u=-\zeta_{h}$. If any of the assumptions A1/A2 is satisfied, by (48), we have

$$
\left\|u_{h}-P_{h}^{\star} u\right\| \leq\left(C_{4} h^{4 k}+\sum_{I_{j} \subset I^{N U}} C_{2} h^{4 k-1}\right)^{\frac{1}{2}} .
$$

When $k \geq 3$, to relax the regularity requirement, we follow the same steps in Lemma 4.2 , and change the definition of $u_{I}$ to $u_{I}=P_{h}^{\star} u-w_{1}$. Then $\epsilon_{h}=u-u_{I}, \zeta_{h}=u_{I}-u_{h}$ and we obtain

$$
\left|a\left(\epsilon_{h}, v_{h}\right)\right| \leq C_{2,1} h^{k+3}\left\|v_{h}\right\|, \quad \forall v_{h} \in V_{h}^{k} .
$$

By the estimates above, (43) and the error equation, we obtain

$$
\frac{d}{d t}\left\|\zeta_{h}\right\|^{2} \leq 2\left\|\left(w_{1}\right)_{t}\right\|\left\|\zeta_{h}\right\| \leq C_{2,1} h^{k+3}
$$

By Gronwall's inequality,

$$
\left\|\zeta_{h}\right\| \leq C_{2,1} t h^{k+3}+\left\|\left.\left(\zeta_{h}\right)\right|_{t=0}\right\|=C_{2,1} t h^{k+3}+\left\|\left.w_{1}\right|_{t=0}\right\| \leq C_{2,1}(1+t) h^{k+3}, \quad \forall t \in\left(0, T_{e}\right],
$$


where the initial numerical discretization is used in the first equality. Since $u_{h}-P_{h}^{\star} u=$ $-\zeta_{h}-w_{1}$, it follows that $\forall t \in\left(0, T_{e}\right]$,

$$
\left\|u_{h}-P_{h}^{\star} u\right\| \leq\left\|\zeta_{h}\right\|+\left\|w_{1}\right\| \leq C_{2,1}(1+t) h^{k+3} .
$$

Then the proof for (55) is complete.

If any of the assumptions A1/A2/A3 is satisfied, then

$$
\begin{aligned}
E_{u} & \leq\left(\frac{1}{\left|D^{0}\right|} \sum_{x \in D^{0}}\left|\left(u-P_{h}^{\dagger} u\right)(x)\right|^{2}+\left|\left(P_{h}^{\star} u-u_{h}\right)(x)\right|^{2}+\left|\left(P_{h}^{\star} u-P_{h}^{\dagger} u\right)(x)\right|^{2}\right)^{\frac{1}{2}} \\
& \leq C h^{k+2}|u|_{W^{k+2,2}(I)}+C\left\|P_{h}^{\star} u-u_{h}\right\|+C\left\|P_{h}^{\star} u-P_{h}^{\dagger} u\right\|,
\end{aligned}
$$

where (33), inverse inequality, and (29) are used in the last inequality. Then the estimates for $E_{u}$ is proven by Lemma 3.4 and (55). The estimates for $E_{u_{x}}$ and $E_{u_{x x}}$ can be proven following the same lines.

Remark 4.7. If the initial discretization is taken as $\left.u_{h}\right|_{t=0}=\left.u_{I}\right|_{t=0}$, the theorem above still holds. However, the regularity requirement will be higher.

\subsection{Superconvergence after postprocessing}

In this section, we analyze the superconvergence property of the postprocessed DG solutions for linear Schrödinger equation (1) on uniform mesh by using negative Sobolev norm estimates. The postprocessor was originally introduced in [4, 20] for finite difference and finite element methods, and later applied to DG methods in [13]. The postprocessed solution is computed by convoluting the numerical solution $u_{h}$ with a kernel function $K_{h}^{\nu, l}(x)=\frac{1}{h^{d}} K^{\nu, l}\left(\frac{x}{h}\right)$, where $d$ is the number of spatial dimensions, and $l$ is the index of $H^{-l}$ norm we're trying to estimate later. The convolution kernel has three main properties. First, it has compact support, making post processing computationally advantageous. Second, it preserves polynomials of degree up to $\nu-1$ by convolution, thus the convergence rate is not deteriorated. Third, the kernel $K^{\nu, l}$ is a linear combination of B-splines, which allows us to express the derivatives of kernel by difference quotients (see section 4.1 in [13]).

We give the formula for the convolution kernel when the DG scheme uses approximation space $V_{h}^{k}$ :

$$
K^{2(k+1), k+1}(x)=\sum_{\gamma=-k}^{k} k_{\gamma}^{2(k+1), k+1} \psi^{(k+1)}(x-\gamma),
$$

where $\psi^{(k+1)}$ are the B-spline bases and the computation procedure of coefficients $k_{\gamma}^{2(k+1), k+1}$ can be found in [22]. Then we can define the postprocessed DG solution as

$$
u^{*}=\int_{-\infty}^{\infty} K_{h}^{2(k+1), k+1}(y-x) u_{h}(y) d y .
$$

$u^{*}$ is an "averaged" version of $u_{h}$ such that it is closer as an approximation to the exact solution $u$. Lastly, we define divided difference as

$$
d_{h} v(x)=\frac{1}{h}\left(v\left(x+\frac{1}{2} h\right)-v\left(x-\frac{1}{2} h\right)\right) .
$$


Now we are ready to state an approximation result showing the smoothness of $u$ and negative Sobolev norm of divided difference lead to a bound on $u-u^{*}$.

Theorem 4.8 (Bramble and Schatz [4]). Suppose $u^{*}$ is defined in (59) and $K_{h}^{2(k+1), k+1}=$ $\frac{1}{h} K^{2(k+1), k+1}\left(\frac{x}{h}\right)$, where $K^{2(k+1), k+1}$ is a kernel function as defined above. Let $u$ be the exact solution of linear Schrödinger equation (1) satisfying periodic boundary condition, $u \in H^{2 k+2}(I)$. Then for arbitrary time $t \in\left(0, T_{e}\right], h$ sufficiently small, we have

$$
\left\|u-u^{*}\right\| \leq C h^{2 k+2}|u|_{H^{2 k+2}(I)}+\sum_{\alpha \leq k+1}\left\|d_{h}^{\alpha}\left(u-u_{h}\right)\right\|_{H^{-(k+1)}\left(\mathcal{I}_{N}\right)},
$$

where $C$ is independent of $u$ and $h$.

The right hand side of (60) indicates that if $\left\|d_{h}^{\alpha}\left(u-u_{h}\right)\right\|_{H^{-(k+1)}\left(\mathcal{I}_{N}\right)}$ converges at a rate higher than $k+1$, then we have superconvergence property for the postprocessed solution. In what follows, we estimate the negative-norm term following the steps in [13]. First, we introduce a dual problem: find a function $v$ such that $v(\cdot, t)$ is periodic function with period equal to the length of $I$, i.e., $b-a$ for all $t \in\left(0, T_{e}\right]$ and

$$
\begin{aligned}
i v_{t}-v_{x x}=0, & \text { in } \mathbb{R} \times\left(0, T_{e}\right), \\
v\left(x, T_{e}\right)=\Phi(x), & x \in \mathbb{R},
\end{aligned}
$$

where $\Phi$ is an arbitrary function in $\mathcal{C}_{0}^{\infty}(I)$. We use the notation $(\phi, \psi):=\int_{I} \phi \psi d x$ in this section. At final time $T_{e}$,

$$
\begin{aligned}
\left(u\left(T_{e}\right)-u_{h}\left(T_{e}\right), \Phi\right) & =(u, v)\left(T_{e}\right)-\left(u_{h}, v\right)\left(T_{e}\right) \\
& =(u, v)(0)+\int_{0}^{T_{e}}\left\{\left(u, v_{t}\right)+\left(u_{t}, v\right)\right\} d t-\left(u_{h}, v\right)\left(T_{e}\right) \\
& =(u, v)(0)-\left(u_{h}, v\right)(0)-\int_{0}^{T_{e}}\left\{\left(\left(u_{h}\right)_{t}, v\right)+\left(u_{h}, v_{t}\right)\right\} d t \\
& =\left(u-u_{h}, v\right)(0)-\int_{0}^{T_{e}}\left\{\left(\left(u_{h}\right)_{t}, v\right)+\left(u_{h}, v_{t}\right)\right\} d t,
\end{aligned}
$$

where the property $u v_{t}+u_{t} v=0$ is used to obtain the third equality.

The DG solution $u_{h}$ satisfies (44). Therefore, we have $\forall v_{h} \in V_{h}^{k}$

$$
\begin{aligned}
\left(\left(u_{h}\right)_{t}, v\right) & =\left(\left(u_{h}\right)_{t}, v-v_{h}\right)+\left(\left(u_{h}\right)_{t}, v_{h}\right) \\
& =\left(\left(u_{h}\right)_{t}, v-v_{h}\right)+i A\left(u_{h}, v_{h}\right) \\
& =\left(\left(u_{h}\right)_{t}, v-v_{h}\right)-i A\left(u_{h}, v-v_{h}\right)+i A\left(u_{h}, v\right) .
\end{aligned}
$$

Then we obtain

$$
\left(u\left(T_{e}\right)-u_{h}\left(T_{e}\right), \Phi\right)=\Theta_{M}+\Theta_{N}+\Theta_{C}
$$

where

$$
\begin{aligned}
& \Theta_{M}=\left(u-u_{h}, v\right)(0), \\
& \Theta_{N}=-\int_{0}^{T_{e}}\left\{\left(\left(u_{h}\right)_{t}, v-v_{h}\right)-i A\left(u_{h}, v-v_{h}\right)\right\} d t, \quad \forall v_{h} \in V_{h}^{k}, \\
& \Theta_{C}=-\int_{0}^{T_{e}}\left\{\left(u_{h}, v_{t}\right)+i A\left(u_{h}, v\right)\right\} d t .
\end{aligned}
$$


By choosing the initial numerical discretization $u_{h}(0)=P_{h}^{0} u_{0}$ and $v_{h}=P_{h}^{0} v$, we have $\Theta_{M}=\left(u-u_{h}, v\right)(0)=\left(u-u_{h}, v-v_{h}\right)(0)$ and

$$
\left|\Theta_{M}\right| \leq\left\|\left(u-u_{h}\right)(0)\right\| \cdot\left\|\left(v-v_{h}\right)(0)\right\| \leq C h^{2 k+2}\|u\|_{H^{k+1}(I)}\|v\|_{H^{k+1}(I)} .
$$

Since $v$ is a smooth function, we have

$$
\Theta_{C}=-\int_{0}^{T_{e}}\left\{\left(u_{h}, v_{t}\right)+i\left(u_{h}, v_{x x}\right)\right\} d t=0 .
$$

Choose $v_{h}=P_{h}^{0} v$ and from the symmetry of the operator $A(\cdot, \cdot)$, we get

$$
\begin{aligned}
\left|\Theta_{N}\right| & \left.=\left|\int_{0}^{T_{e}} A\left(u_{h}, v-v_{h}\right) d t\right|=\left|\int_{0}^{T_{e}} A\left(v-v_{h}, u_{h}\right) d t\right|=\mid \int_{0}^{T_{e}} \sum_{j=1}^{N}\left(\widehat{v-v_{h}}\left[\left(u_{h}\right)_{x}\right]-\widetilde{\left(v-v_{h}\right.}\right)_{x}\left[u_{h}\right]\right)\left.\right|_{j+\frac{1}{2}} d t \mid \\
& \left.=\mid \int_{0}^{T_{e}} \sum_{j=1}^{N}\left(\widehat{v-v_{h}}\left[u_{x}-\left(u_{h}\right)_{x}\right]-\widetilde{\left(v-v_{h}\right.}\right)_{x}\left[u-u_{h}\right]\right)\left.\right|_{j+\frac{1}{2}} d t \mid \\
& \leq C T_{e} \max _{t \in\left(0, T_{e}\right]}\left(\left\|u-u_{h}\right\|_{L^{2}\left(\partial \mathcal{I}_{N}\right)}\left\|\left(\widetilde{v-v_{h}}\right)_{x}\right\|_{L^{2}\left(\partial \mathcal{I}_{N}\right)}+\left\|\left(u-u_{h}\right)_{x}\right\|_{L^{2}\left(\partial \mathcal{I}_{N}\right)}\left\|\widehat{v-v_{h}}\right\|_{L^{2}\left(\partial \mathcal{I}_{N}\right)}\right) .
\end{aligned}
$$

By (26),

$$
\begin{aligned}
\left\|u-u_{h}\right\|_{L^{2}\left(\partial \mathcal{I}_{N}\right)} & =\left\|u-P_{h}^{\star} u\right\|_{L^{2}\left(\partial \mathcal{I}_{N}\right)}+\left\|P_{h}^{\star} u-u_{h}\right\|_{L^{2}\left(\partial \mathcal{I}_{N}\right)} \\
& \leq C_{0} h^{k+\frac{1}{2}}+C h^{-\frac{1}{2}}\left\|P_{h}^{\star} u-u_{h}\right\| \leq C_{2} h^{k+\frac{1}{2}},
\end{aligned}
$$

where we have used Lemma 3.2 and Theorem 3.3. Similarly, we have $\left\|u_{x}-\left(u_{h}\right)_{x}\right\|_{L^{2}\left(\partial \mathcal{I}_{N}\right)} \leq$ $C_{2} h^{k-\frac{1}{2}}$. By the property of $L^{2}$ projection $h^{\frac{3}{2}}\left\|v_{x}-\left(v_{h}\right)_{x}\right\|_{L^{2}\left(\partial \mathcal{I}_{N}\right)}+h\left\|v_{x}-\left(v_{h}\right)_{x}\right\|+h^{\frac{1}{2}} \| v-$ $v_{h}\left\|_{L^{2}\left(\partial \mathcal{I}_{N}\right)}+\right\| v-v_{h}\left\|\leq C h^{k+1}\right\| v \|_{H^{k+1}(I)}$. Then it is straightforward that for scale invariant fluxes

$$
\left\|\widehat{v-v_{h}}\right\|_{L^{2}\left(\partial \mathcal{I}_{N}\right)} \leq C h^{k+\frac{1}{2}}\|v\|_{H^{k+1}(I)}, \quad \|\left(\widetilde{\left.v-v_{h}\right)_{x}}\left\|_{L^{2}\left(\partial \mathcal{I}_{N}\right)} \leq C h^{k-\frac{1}{2}}\right\| v \|_{H^{k+1}(I)} .\right.
$$

Therefore, we have

$$
\left|\Theta_{N}\right| \leq C_{2} h^{2 k}\|v\|_{H^{k+1}(I)} .
$$

Combine the above three estimate and the fact $\|v\|_{H^{k+1}(I)}=\|\Phi\|_{H^{k+1}(I)}$, we have

$$
\left\|u\left(T_{e}\right)-u_{h}\left(T_{e}\right)\right\|_{H^{-(k+1)}(I)} \leq C_{2} h^{2 k} .
$$

Since we consider $u_{h}$ with optimal error estimates on uniform mesh with mesh size $h$, then the divided difference $d_{h}^{\alpha} u$ satisfies the linear Schrödinger but with initial data $d_{h}^{\alpha} u_{0}, \alpha \leq k+1$ on shifted mesh. Similarly, $d_{h}^{\alpha} u_{h}$ also satisfies the DG scheme (4) but with shifted mesh and initial numerical discretization $d_{h}^{\alpha} u_{h}=P_{h}^{0} d_{h}^{\alpha} u_{0}$. Then by the same proof for $u-u_{h}$ above,

$$
\left\|d_{h}^{\alpha}\left(u-u_{h}\right)\left(T_{e}\right)\right\|_{H^{-(k+1)}(I)} \leq C_{2+\alpha} h^{2 k},
$$

where we used Taylor expansion to estimate $d_{h}^{\alpha} u$ to obtain the last inequality.

The following theorem is a result of (63) and Theorem 4.8.

Theorem 4.9. Let $u_{h}$ be the UWDG solution of (4), suppose the conditions in Theorem 4.8 and any of the assumptions A1/A2/A3 is satisfied, then on a uniform mesh

$$
\left\|u\left(T_{e}\right)-u^{*}\left(T_{e}\right)\right\| \leq C_{k+3} h^{2 k} .
$$




\section{$5 \quad$ Numerical Experiments}

In this section, we provide numerical tests demonstrating superconvergence properties. In the proof, we see that the initial value of $u_{h}$ matters in estimating $\left\|u_{h}-u_{I}\right\|$, thus will impact the superconvergence estimation for $E_{f}$ and $E_{f_{x}}$. Therefore, in our numerical tests, we apply two types of initial discretization for $u_{h}$. For computing the postprocessed solution $u^{*}$, we use the standard $L^{2}$ projection $P_{h}^{0} u$ as numerical initialization to demonstrate the convergence enhancement ability of postprocessor. For verifying other superconvergence quantities, we apply the initial condition $\left.u_{h}\right|_{t=0}=\left.u_{I}\right|_{t=0}$. In order not to deteriorate the high order convergence rates, for temporal discretization, we use explicit Runge-Kutta fourth order method with $d t=c \cdot h^{2.5}, c=0.05$ when $k=2$ and $c=0.01$ when $k=3,4$.

Example 5.1. We compute (10) on $[0,2 \pi]$ with exact solution $u(x, t)=\exp (i 3(x-3 t))$ using $D G$ scheme (4). We verify the results with several flux parameters.

In the following tables, we show the convergence rate for quantities $E_{f}, E_{f_{x}}, E_{c}, E_{u}, E_{u_{x}}, E_{u_{x x}}$ as defined in (50) and (56) as well as

$$
E^{*}=\left\|u-u^{*}\right\|, \quad E_{P}=\left\|u_{h}-P_{h}^{\star} u\right\|,
$$

which represent the error after postprocessing, and the error between numerical solution and the projected exact solution $P_{h}^{\star} u$. In addition, we test the superconvergence of the intermediate quantities $\zeta_{h}=P_{h}^{\star} u-w-u_{h}$ as in Lemma 4.3, and introduce the following notations:

$$
E_{\left[\zeta_{h}\right]}=\left(\frac{1}{N} \sum_{j=1}^{N}\left|\left[\zeta_{h}\right]\right|_{j+\frac{1}{2}}^{2}\right)^{\frac{1}{2}}, \quad E_{\left[\left(\zeta_{h}\right)_{x}\right]}=\left(\frac{1}{N} \sum_{j=1}^{N}\left|\left[\left(\zeta_{h}\right)_{x}\right]\right|_{j+\frac{1}{2}}^{2}\right)^{\frac{1}{2}} .
$$

The numerical fluxes we tested include

1. Tables 2, 3: A1 parameters, alternating flux, $\alpha_{1}=0.5, \beta_{1}=\beta_{2}=0$, with nonuniform mesh;

2. Tables 4, 5: A1 parameters, a scale invariant flux, $\alpha_{1}=0.3, \beta_{1}=\frac{0.4}{h}, \beta_{2}=0.4 h$, with nonuniform mesh;

3. Tables 6. 7): A2 parameters, central flux, $\alpha_{1}=\beta_{1}=\beta_{2}=0$, with uniform mesh;

4. Tables 8, A3 parameters, $\alpha_{1}=0.25, \beta_{2}=0, \beta_{1}=\frac{2}{h}, \frac{5}{h}, \frac{9}{h}$ for $k=2,3,4$, respectively, with uniform mesh;

5. Table 9, all parameters mentioned above, with uniform mesh,

where the nonuniform mesh is generated by perturbing the location of the nodes of a uniform mesh by $10 \%$ of mesh size.

We first verify the results in Theorems 4.5, 4.6 by examining Tables 2, 4, 6, 8, where the parameters satisfy assumption A1, A1, A2, A3, respectively. We observe that the scheme can achieve at least the theoretical order of convergence for the quantities in these two theorems. To be more specific, $E_{P}$ shows $(k+\min (3, k))$-th order of convergence. $E_{u}, E_{u_{x}}, E_{u_{x x}}$ are shown to have $(k+2)$-th, $(k+1)$-th and $k$-th order of convergence, respectively. Note 
that when $k=2$, in Tables 4 and 8 , there are situations when no superconvergence point exists. This finding shows an evidence to the assertion that $D^{s}$ defined in (33) could be empty sets. The order of convergence for $E_{f},\left(E_{f}\right)_{x}, E_{c}$ in all tables are $2 k$. In addition, Table 8 shows that when $k$ is even and assumption A3 is satisfied, the convergence order for all quantities are the same as when any of assumption A1/A2 is satisfied, which is one order higher than the estimates in Theorems 4.5, 4.6. In Tables 2 and 4, we used nonuniform mesh in numerical test. The quantities tested have similar order of convergence compared to the order of convergence on uniform mesh. Another interesting observation is the order of convergence of $E_{f_{x}}$. Our numerical tests show that $E_{f_{x}}$ converges at an order of $2 k$ for all four sets of parameters, which is at least one order higher than the estimates in Theorem 4.5 .

Next, we test the order of convergence for quantities in Lemma 4.3, In Tables 3 and 5 , we observe clean convergence order of $2 k-1,2 k+1,2 k$ for $\left\|\left(\zeta_{h}\right)_{x x}\right\|, E_{\left[\zeta_{h}\right]}, E_{\left[\left(\zeta_{h}\right)_{x}\right]}$ when $k$ is even and $2 k, 2 k+2,2 k+1$ for these three quantities when $k$ is odd. In Table 7 , the order of convergence has some fluctuation, but the quantities are shown to have the same order of convergence as those in Tables 3 and 5. These convergence rates are consistent with the results in Lemma 4.3 .

Lastly, we test the order of convergence for $E^{*}$ on uniform mesh for the four sets of parameters. Table 9 shows that $E^{*}$ has a convergence rate of at least $2 k$, and can go up to $2 k+2$. Similar higher order of convergence behaviors exists in the literature [13, 22].

Table 2: Example 5.1 Error table when using alternating flux on nonuniform mesh. Ending time $T_{e}=1, x \in[0,2 \pi]$.

\begin{tabular}{|c|c|c|c|c|c|c|c|c|c|c|c|c|c|c|c|c|c|}
\hline & $\mathrm{N}$ & $L^{2}$ error & order & $E_{P}$ & order & $E_{u_{x x}}$ & order & $E_{u_{x}}$ & order & $E_{u}$ & order & $E_{f}$ & order & $E_{f_{x}}$ & order & $E_{c}$ & order \\
\hline \multirow{5}{*}{$P^{2}$} & 10 & $2.68 \mathrm{E}-01$ & - & $2.53 \mathrm{E}-01$ & - & $2.36 \mathrm{E}+00$ & - & $8.92 \mathrm{E}-01$ & - & $2.83 \mathrm{E}-01$ & - & $2.79 \mathrm{E}-01$ & 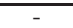 & $9.10 \mathrm{E}-01$ & - & $5.00 \mathrm{E}-01$ & - \\
\hline & 20 & $2.68 \mathrm{E}-02$ & 3.32 & $2.47 \mathrm{E}-02$ & 3.36 & $2.78 \mathrm{E}-01$ & 3.09 & $7.43 \mathrm{E}-02$ & 3.59 & $2.30 \mathrm{E}-02$ & 3.62 & $2.31 \mathrm{E}-02$ & 3.60 & $6.94 \mathrm{E}-02$ & 3.71 & $4.48 \mathrm{E}-02$ & 3.48 \\
\hline & 40 & $2.00 \mathrm{E}-03$ & 3.75 & $1.42 \mathrm{E}-03$ & 4.12 & $6.02 \mathrm{E}-02$ & 2.21 & $5.29 \mathrm{E}-03$ & 3.81 & $1.47 \mathrm{E}-03$ & 3.97 & $1.46 \mathrm{E}-03$ & 3.98 & $4.45 \mathrm{E}-03$ & 3.96 & $2.91 \mathrm{E}-03$ & 3.94 \\
\hline & 80 & $1.91 \mathrm{E}-04$ & 3.39 & $9.22 \mathrm{E}-05$ & 3.95 & $1.50 \mathrm{E}-02$ & 2.00 & $3.83 \mathrm{E}-04$ & 3.79 & $9.12 \mathrm{E}-05$ & 4.01 & $9.11 \mathrm{E}-05$ & 4.01 & $2.75 \mathrm{E}-04$ & 4.02 & $1.82 \mathrm{E}-04$ & 4.00 \\
\hline & 160 & $2.19 \mathrm{E}-05$ & 3.12 & $5.83 \mathrm{E}-06$ & 3.98 & $3.78 \mathrm{E}-03$ & 1.99 & $3.24 \mathrm{E}-05$ & 3.56 & $5.78 \mathrm{E}-06$ & 3.98 & $5.77 \mathrm{E}-06$ & 3.98 & $1.74 \mathrm{E}-05$ & 3.98 & $1.15 \mathrm{E}-05$ & 3.98 \\
\hline \multirow{5}{*}{$P^{3}$} & 10 & $1.02 \mathrm{E}-02$ & - & $7.51 \mathrm{E}-03$ & - & $1.60 \mathrm{E}-01$ & - & $2.50 \mathrm{E}-02$ & - & $6.81 \mathrm{E}-03$ & - & $6.78 \mathrm{E}-03$ & - & $1.98 \mathrm{E}-02$ & 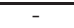 & $1.16 \mathrm{E}-02$ & - \\
\hline & 20 & $5.65 \mathrm{E}-04$ & 4.18 & $1.24 \mathrm{E}-04$ & 5.93 & $2.00 \mathrm{E}-02$ & 3.00 & $8.23 \mathrm{E}-04$ & 4.93 & $1.40 \mathrm{E}-04$ & 5.61 & $1.36 \mathrm{E}-04$ & 5.64 & $4.08 \mathrm{E}-04$ & 5.60 & $2.64 \mathrm{E}-04$ & 5.45 \\
\hline & 40 & $2.94 \mathrm{E}-05$ & 4.26 & $2.13 \mathrm{E}-06$ & 5.86 & $2.43 \mathrm{E}-03$ & 3.04 & $3.83 \mathrm{E}-05$ & 4.42 & $2.16 \mathrm{E}-06$ & 6.01 & $2.02 \mathrm{E}-06$ & 6.07 & $6.06 \mathrm{E}-06$ & 6.07 & $4.03 \mathrm{E}-06$ & 6.03 \\
\hline & 80 & $1.87 \mathrm{E}-06$ & 3.98 & $3.02 \mathrm{E}-08$ & 6.14 & $2.99 \mathrm{E}-04$ & 3.02 & $2.29 \mathrm{E}-06$ & 4.06 & $3.73 \mathrm{E}-08$ & 5.86 & $3.05 \mathrm{E}-08$ & 6.05 & $9.13 \mathrm{E}-08$ & 6.05 & $6.11 \mathrm{E}-08$ & 6.04 \\
\hline & 160 & $1.18 \mathrm{E}-07$ & 3.98 & $4.57 \mathrm{E}-10$ & 6.05 & $3.76 \mathrm{E}-05$ & 2.99 & $1.45 \mathrm{E}-07$ & 3.98 & $8.04 \mathrm{E}-10$ & 5.53 & $4.59 \mathrm{E}-10$ & 6.05 & $1.38 \mathrm{E}-09$ & 6.05 & $9.25 \mathrm{E}-10$ & 6.05 \\
\hline \multirow{5}{*}{$P^{4}$} & 10 & $6.45 \mathrm{E}-04$ & - & $8.76 \mathrm{E}-05$ & - & $1.79 \mathrm{E}-02$ & $\begin{array}{lll}- & - \\
- & -1\end{array}$ & $8.67 \mathrm{E}-04$ & - & $1.09 \mathrm{E}-04$ & - & $9.69 \mathrm{E}-05$ & 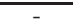 & $2.83 \mathrm{E}-04$ & 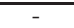 & $1.62 \mathrm{E}-04$ & - \\
\hline & 20 & $2.06 \mathrm{E}-05$ & 4.97 & $6.12 \mathrm{E}-07$ & 7.16 & $1.25 \mathrm{E}-03$ & 3.84 & $3.06 \mathrm{E}-05$ & 4.82 & $1.04 \mathrm{E}-06$ & 6.72 & $5.02 \mathrm{E}-07$ & 7.59 & $1.57 \mathrm{E}-06$ & 7.49 & $9.69 \mathrm{E}-07$ & 7.39 \\
\hline & 40 & $6.83 \mathrm{E}-07$ & 4.91 & $2.52 \mathrm{E}-09$ & 7.92 & $7.45 \mathrm{E}-05$ & 4.06 & $9.00 \mathrm{E}-07$ & 5.09 & $1.28 \mathrm{E}-08$ & 6.34 & $1.76 \mathrm{E}-09$ & 8.16 & $5.26 \mathrm{E}-09$ & 8.22 & $3.50 \mathrm{E}-09$ & 8.11 \\
\hline & 80 & $2.04 \mathrm{E}-08$ & 5.07 & $1.55 \mathrm{E}-11$ & 7.35 & $4.70 \mathrm{E}-06$ & 3.99 & $2.78 \mathrm{E}-08$ & 5.02 & $1.94 \mathrm{E}-10$ & 6.04 & $6.99 \mathrm{E}-12$ & 7.97 & $2.09 \mathrm{E}-11$ & 7.97 & $1.40 \mathrm{E}-11$ & 7.97 \\
\hline & 160 & $6.10 \mathrm{E}-10$ & 5.06 & $1.02 \mathrm{E}-13$ & 7.24 & $2.85 \mathrm{E}-07$ & 4.04 & $8.34 \mathrm{E}-10$ & 5.06 & $2.85 \mathrm{E}-12$ & 6.09 & $2.79 \mathrm{E}-14$ & 7.97 & $5.55 \mathrm{E}-13$ & 5.23 & $5.07 \mathrm{E}-14$ & 8.10 \\
\hline
\end{tabular}

Table 3: Example 5.1 Error table for intermediate quantities when using alternating flux on nonuniform mesh. Ending time $T_{e}=1, x \in[0,2 \pi]$.

\begin{tabular}{|c|c|c|c|c|c|c|c|c|c|}
\hline & $\mathrm{N}$ & $\left\|\zeta_{h}\right\|$ error & order & $\left\|\left(\zeta_{h}\right)_{x x}\right\|$ & order & $E_{\left[\zeta_{h}\right]}$ & order & $E_{\left[\left(\zeta_{h}\right)_{x}\right]}$ & order \\
\hline \multirow{5}{*}{$P^{2}$} & 10 & $3.96 \mathrm{E}-01$ & - & $3.02 \mathrm{E}+00$ & - & $4.51 \mathrm{E}-02$ & - & $3.37 \mathrm{E}-01$ & - \\
& 20 & $3.28 \mathrm{E}-02$ & 3.60 & $2.23 \mathrm{E}-01$ & 3.76 & $1.57 \mathrm{E}-03$ & 4.84 & $1.82 \mathrm{E}-02$ & 4.21 \\
& 40 & $2.08 \mathrm{E}-03$ & 3.98 & $1.42 \mathrm{E}-02$ & 3.98 & $4.21 \mathrm{E}-05$ & 5.22 & $9.42 \mathrm{E}-04$ & 4.28 \\
& 80 & $1.29 \mathrm{E}-04$ & 4.01 & $7.95 \mathrm{E}-04$ & 4.15 & $1.17 \mathrm{E}-06$ & 5.16 & $5.50 \mathrm{E}-05$ & 4.10 \\
& 160 & $8.17 \mathrm{E}-06$ & 3.98 & $9.54 \mathrm{E}-05$ & 3.06 & $3.83 \mathrm{E}-08$ & 4.94 & $3.55 \mathrm{E}-06$ & 3.96 \\
\hline \multirow{4}{*}{$P^{3}$} & 10 & $9.54 \mathrm{E}-03$ & - & $8.83 \mathrm{E}-02$ & - & $7.52 \mathrm{E}-05$ & - & $1.31 \mathrm{E}-03$ & - \\
& 20 & $1.93 \mathrm{E}-04$ & 5.63 & $1.76 \mathrm{E}-03$ & 5.65 & $1.75 \mathrm{E}-07$ & 8.75 & $6.16 \mathrm{E}-06$ & 7.74 \\
& 40 & $2.86 \mathrm{E}-06$ & 6.08 & $2.59 \mathrm{E}-05$ & 6.09 & $3.42 \mathrm{E}-10$ & 9.00 & $2.45 \mathrm{E}-08$ & 7.97 \\
& 80 & $4.31 \mathrm{E}-08$ & 6.05 & $3.91 \mathrm{E}-07$ & 6.05 & $1.45 \mathrm{E}-12$ & 7.88 & $2.71 \mathrm{E}-10$ & 6.50 \\
& 160 & $6.87 \mathrm{E}-10$ & 5.97 & $6.19 \mathrm{E}-09$ & 5.98 & $6.76 \mathrm{E}-15$ & 7.74 & $2.59 \mathrm{E}-12$ & 6.71 \\
\hline \multirow{5}{*}{$P^{4}$} & 10 & $1.35 \mathrm{E}-04$ & - & $1.41 \mathrm{E}-03$ & - & $4.97 \mathrm{E}-07$ & - & $2.81 \mathrm{E}-05$ & - \\
& 20 & $7.10 \mathrm{E}-07$ & 7.57 & $8.60 \mathrm{E}-06$ & 7.36 & $1.20 \mathrm{E}-09$ & 8.69 & $9.88 \mathrm{E}-08$ & 8.15 \\
& 40 & $2.50 \mathrm{E}-09$ & 8.15 & $4.24 \mathrm{E}-08$ & 7.66 & $2.56 \mathrm{E}-12$ & 8.88 & $2.90 \mathrm{E}-10$ & 8.41 \\
& 80 & $9.90 \mathrm{E}-12$ & 7.98 & $2.55 \mathrm{E}-10$ & 7.38 & $2.98 \mathrm{E}-15$ & 9.74 & $7.48 \mathrm{E}-13$ & 8.60 \\
& 160 & $3.58 \mathrm{E}-14$ & 8.11 & $2.22 \mathrm{E}-12$ & 6.85 & $8.90 \mathrm{E}-18$ & 8.39 & $5.46 \mathrm{E}-15$ & 7.10 \\
\hline
\end{tabular}


Table 4: Example 5.1 Error table when using flux parameters: $\alpha_{1}=0.3, \beta_{1}=\frac{0.4}{h}, \beta_{2}=0.4 h$ on nonuniform mesh. Ending time $T_{e}=1, x \in[0,2 \pi]$.

\begin{tabular}{|c|c|c|c|c|c|c|c|c|c|c|c|c|c|c|c|c|c|}
\hline & $\mathrm{N}$ & $L^{2}$ error & order & $E_{P}$ & order & $E_{u_{x x}}$ & order & $E_{u_{x}}$ & order & $E_{u}$ & order & $E_{f}$ & order & $E_{f_{x}}$ & order & $E_{c}$ & order \\
\hline \multirow{5}{*}{$P^{2}$} & 40 & $1.66 \mathrm{E}-02$ & 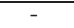 & $1.28 \mathrm{E}-02$ & 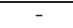 & $2.38 \mathrm{E}-01$ & - & DNE & - & $1.26 \mathrm{E}-02$ & - & $1.26 \mathrm{E}-02$ & 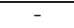 & $3.83 \mathrm{E}-02$ & - & $2.49 \mathrm{E}-02$ & 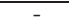 \\
\hline & 80 & $1.50 \mathrm{E}-03$ & 3.47 & $7.46 \mathrm{E}-04$ & 4.10 & $2.52 \mathrm{E}-02$ & 3.24 & DNE & - & $7.43 \mathrm{E}-04$ & 4.08 & $7.42 \mathrm{E}-04$ & 4.08 & $2.28 \mathrm{E}-03$ & 4.07 & $1.48 \mathrm{E}-03$ & 4.07 \\
\hline & 160 & $1.70 \mathrm{E}-04$ & 3.14 & $4.91 \mathrm{E}-05$ & 3.92 & $4.51 \mathrm{E}-03$ & 2.48 & DNE & - & $4.82 \mathrm{E}-05$ & 3.95 & $4.82 \mathrm{E}-05$ & 3.95 & $1.47 \mathrm{E}-04$ & 3.95 & $9.63 \mathrm{E}-05$ & 3.94 \\
\hline & 320 & $2.16 \mathrm{E}-05$ & 2.98 & $3.12 \mathrm{E}-06$ & 3.98 & $8.89 \mathrm{E}-04$ & 2.34 & DNE & - & $3.09 \mathrm{E}-06$ & 3.96 & $3.09 \mathrm{E}-06$ & 3.96 & $9.39 \mathrm{E}-06$ & 3.97 & $6.18 \mathrm{E}-06$ & 3.96 \\
\hline & 640 & $2.64 \mathrm{E}-06$ & 3.03 & $1.96 \mathrm{E}-07$ & 3.99 & $2.00 \mathrm{E}-04$ & 2.15 & DNE & - & $1.94 \mathrm{E}-07$ & 3.99 & $1.94 \mathrm{E}-07$ & 3.99 & $5.86 \mathrm{E}-07$ & 4.00 & $3.88 \mathrm{E}-07$ & 3.99 \\
\hline \multirow{5}{*}{$P^{3}$} & 10 & $2.08 \mathrm{E}-02$ & - & $1.57 \mathrm{E}-02$ & - & $2.00 \mathrm{E}-01$ & $\begin{array}{lll}- & - \\
\end{array}$ & $4.47 \mathrm{E}-02$ & - & $1.44 \mathrm{E}-02$ & - & $1.41 \mathrm{E}-02$ & $\begin{array}{ll}- \\
-\end{array}$ & $4.38 \mathrm{E}-02$ & - & $2.49 \mathrm{E}-02$ & 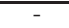 \\
\hline & 20 & $1.14 \mathrm{E}-03$ & 4.19 & $2.75 \mathrm{E}-04$ & 5.84 & $2.23 \mathrm{E}-02$ & 3.17 & $1.42 \mathrm{E}-03$ & 4.98 & $3.08 \mathrm{E}-04$ & 5.54 & $3.05 \mathrm{E}-04$ & 5.53 & $9.17 \mathrm{E}-04$ & 5.58 & $5.92 \mathrm{E}-04$ & 5.39 \\
\hline & 40 & $5.91 \mathrm{E}-05$ & 4.27 & $4.83 \mathrm{E}-06$ & 5.83 & $2.72 \mathrm{E}-03$ & 3.03 & $6.57 \mathrm{E}-05$ & 4.43 & $4.70 \mathrm{E}-06$ & 6.04 & $4.56 \mathrm{E}-06$ & 6.06 & $1.37 \mathrm{E}-05$ & 6.06 & $9.12 \mathrm{E}-06$ & 6.02 \\
\hline & 80 & $3.75 \mathrm{E}-06$ & 3.98 & $6.81 \mathrm{E}-08$ & 6.15 & $3.36 \mathrm{E}-04$ & 3.02 & $3.91 \mathrm{E}-06$ & 4.07 & $7.56 \mathrm{E}-08$ & 5.96 & $6.88 \mathrm{E}-08$ & 6.05 & $2.06 \mathrm{E}-07$ & 6.05 & $1.38 \mathrm{E}-07$ & 6.04 \\
\hline & 160 & $2.39 \mathrm{E}-07$ & 3.97 & $1.09 \mathrm{E}-09$ & 5.96 & $4.23 \mathrm{E}-05$ & 2.99 & $2.47 \mathrm{E}-07$ & 3.98 & $1.03 \mathrm{E}-09$ & 6.20 & $1.10 \mathrm{E}-09$ & 5.97 & $3.29 \mathrm{E}-09$ & 5.97 & $2.21 \mathrm{E}-09$ & 5.97 \\
\hline \multirow{5}{*}{$P^{4}$} & 10 & $9.72 \mathrm{E}-04$ & - & $1.48 \mathrm{E}-04$ & - & $1.93 \mathrm{E}-02$ & - & $1.32 \mathrm{E}-03$ & - & $1.77 \mathrm{E}-04$ & - & $1.65 \mathrm{E}-04$ & - & $4.69 \mathrm{E}-04$ & - & $2.73 \mathrm{E}-04$ & - \\
\hline & 20 & $3.17 \mathrm{E}-05$ & 4.94 & $1.04 \mathrm{E}-06$ & 7.16 & $1.37 \mathrm{E}-03$ & 3.82 & $4.63 \mathrm{E}-05$ & 4.83 & $1.56 \mathrm{E}-06$ & 6.83 & $8.66 \mathrm{E}-07$ & 7.57 & $2.52 \mathrm{E}-06$ & 7.54 & $1.66 \mathrm{E}-06$ & 7.36 \\
\hline & 40 & $1.05 \mathrm{E}-06$ & 4.91 & $4.15 \mathrm{E}-09$ & 7.97 & $8.18 \mathrm{E}-05$ & 4.06 & $1.35 \mathrm{E}-06$ & 5.10 & $1.83 \mathrm{E}-08$ & 6.41 & $3.04 \mathrm{E}-09$ & 8.15 & $8.85 \mathrm{E}-09$ & 8.15 & $6.02 \mathrm{E}-09$ & 8.11 \\
\hline & 80 & $3.14 \mathrm{E}-08$ & 5.07 & $2.51 \mathrm{E}-11$ & 7.37 & $5.16 \mathrm{E}-06$ & 3.99 & $4.27 \mathrm{E}-08$ & 4.99 & $2.82 \mathrm{E}-10$ & 6.02 & $1.21 \mathrm{E}-11$ & 7.98 & $3.76 \mathrm{E}-11$ & 7.88 & $2.41 \mathrm{E}-11$ & 7.97 \\
\hline & 160 & $9.39 \mathrm{E}-10$ & 5.06 & $1.63 \mathrm{E}-13$ & 7.27 & 3.13E-07 & 4.04 & $1.27 \mathrm{E}-09$ & 5.07 & $4.12 \mathrm{E}-12$ & 6.10 & $4.41 \mathrm{E}-14$ & 8.10 & $1.30 \mathrm{E}-13$ & 8.18 & $8.73 \mathrm{E}-14$ & 8.11 \\
\hline
\end{tabular}

Table 5: Example 5.1 Error table for intermediate quantities when using flux parameters: $\alpha_{1}=0.3, \beta_{1}=\frac{0.4}{h}, \beta_{2}=0.4 h$ on nonuniform mesh. Ending time $T_{e}=1, x \in[0,2 \pi]$.

\begin{tabular}{|c|c|c|c|c|c|c|c|c|c|}
\hline & $\mathrm{N}$ & $\left\|\zeta_{h}\right\|$ error & order & $\left\|\left(\zeta_{h}\right)_{x x}\right\|$ & order & $E_{\left[\zeta_{h}\right]}$ & order & $E_{\left[\left(\zeta_{h}\right)_{x}\right]}$ & order \\
\hline \multirow{5}{*}{$P^{2}$} & 40 & $1.46 \mathrm{E}-02$ & - & $2.67 \mathrm{E}-01$ & - & $2.55 \mathrm{E}-03$ & - & $2.13 \mathrm{E}-02$ & - \\
& 80 & $9.35 \mathrm{E}-04$ & 3.97 & $2.57 \mathrm{E}-02$ & 3.38 & $7.74 \mathrm{E}-05$ & 5.04 & $1.25 \mathrm{E}-03$ & 4.09 \\
& 160 & $5.96 \mathrm{E}-05$ & 3.97 & $2.86 \mathrm{E}-03$ & 3.17 & $2.52 \mathrm{E}-06$ & 4.94 & $7.56 \mathrm{E}-05$ & 4.05 \\
& 320 & $3.76 \mathrm{E}-06$ & 3.99 & $3.30 \mathrm{E}-04$ & 3.11 & $7.74 \mathrm{E}-08$ & 5.02 & $4.76 \mathrm{E}-06$ & 3.99 \\
& 640 & $2.38 \mathrm{E}-07$ & 3.98 & $4.25 \mathrm{E}-05$ & 2.96 & $2.57 \mathrm{E}-09$ & 4.91 & $3.19 \mathrm{E}-07$ & 3.90 \\
\hline \multirow{5}{*}{$P^{3}$} & 10 & $2.02 \mathrm{E}-02$ & - & $1.78 \mathrm{E}-01$ & - & $5.73 \mathrm{E}-04$ & - & $1.15 \mathrm{E}-03$ & - \\
& 20 & $4.31 \mathrm{E}-04$ & 5.55 & $3.90 \mathrm{E}-03$ & 5.52 & $1.09 \mathrm{E}-06$ & 9.04 & $5.48 \mathrm{E}-06$ & 7.71 \\
& 40 & $6.46 \mathrm{E}-06$ & 6.06 & $5.83 \mathrm{E}-05$ & 6.06 & $3.27 \mathrm{E}-09$ & 8.38 & $2.99 \mathrm{E}-08$ & 7.52 \\
& 80 & $9.72 \mathrm{E}-08$ & 6.05 & $8.76 \mathrm{E}-07$ & 6.06 & $9.55 \mathrm{E}-12$ & 8.42 & $1.84 \mathrm{E}-10$ & 7.34 \\
& 160 & $1.55 \mathrm{E}-09$ & 5.97 & $1.40 \mathrm{E}-08$ & 5.97 & $4.28 \mathrm{E}-14$ & 7.80 & $1.56 \mathrm{E}-12$ & 6.89 \\
\hline \multirow{5}{*}{$P^{4}$} & 10 & $2.27 \mathrm{E}-04$ & - & $2.23 \mathrm{E}-03$ & - & $1.03 \mathrm{E}-06$ & - & $2.06 \mathrm{E}-06$ & - \\
& 20 & $1.22 \mathrm{E}-06$ & 7.54 & $1.30 \mathrm{E}-05$ & 7.42 & $4.13 \mathrm{E}-09$ & 7.96 & $1.66 \mathrm{E}-08$ & 6.96 \\
& 40 & $4.30 \mathrm{E}-09$ & 8.15 & $5.93 \mathrm{E}-08$ & 7.78 & $1.01 \mathrm{E}-11$ & 8.67 & $6.95 \mathrm{E}-11$ & 7.90 \\
& 80 & $1.71 \mathrm{E}-11$ & 7.98 & $4.66 \mathrm{E}-10$ & 6.99 & $1.82 \mathrm{E}-14$ & 9.12 & $2.33 \mathrm{E}-13$ & 8.22 \\
& 160 & $6.17 \mathrm{E}-14$ & 8.11 & $3.19 \mathrm{E}-12$ & 7.19 & $3.61 \mathrm{E}-17$ & 8.98 & $8.34 \mathrm{E}-16$ & 8.13 \\
\hline
\end{tabular}

Table 6: Example 5.1 Error table when using central flux on uniform mesh. Ending time $T_{e}=1, x \in[0,2 \pi]$.

\begin{tabular}{|c|c|c|c|c|c|c|c|c|c|c|c|c|c|c|c|c|c|}
\hline & $\mathrm{N}$ & $L^{2}$ error & order & $E_{P}$ & order & $E_{u_{x x}}$ & order & $E_{u_{x}}$ & order & $E_{u}$ & order & $E_{f}$ & order & $E_{f_{x}}$ & order & $E_{c}$ & order \\
\hline \multirow{5}{*}{$P^{2}$} & 40 & $4.20 \mathrm{E}-03$ & - & $3.21 \mathrm{E}-03$ & & $4.86 \mathrm{E}-01$ & - & $3.39 \mathrm{E}-02$ & - & $3.24 \mathrm{E}-03$ & - & $3.21 \mathrm{E}-03$ & 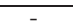 & $9.58 \mathrm{E}-03$ & 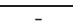 & $6.36 \mathrm{E}-03$ & - \\
\hline & 80 & $4.31 \mathrm{E}-04$ & 3.29 & $2.23 \mathrm{E}-04$ & 3.85 & $1.33 \mathrm{E}-01$ & 1.87 & $4.49 \mathrm{E}-03$ & 2.92 & $2.25 \mathrm{E}-04$ & 3.85 & $2.23 \mathrm{E}-04$ & 3.85 & $6.86 \mathrm{E}-04$ & 3.80 & $4.44 \mathrm{E}-04$ & 3.84 \\
\hline & 160 & $4.92 \mathrm{E}-05$ & 3.13 & $1.43 \mathrm{E}-05$ & 3.96 & $3.41 \mathrm{E}-02$ & 1.97 & $5.69 \mathrm{E}-04$ & 2.98 & $1.45 \mathrm{E}-05$ & 3.96 & $1.43 \mathrm{E}-05$ & 3.96 & $3.90 \mathrm{E}-05$ & 4.14 & $2.86 \mathrm{E}-05$ & 3.96 \\
\hline & 320 & $5.99 \mathrm{E}-06$ & 3.04 & $9.01 \mathrm{E}-07$ & 3.99 & $8.57 \mathrm{E}-03$ & 1.99 & $7.14 \mathrm{E}-05$ & 2.99 & $9.10 \mathrm{E}-07$ & 3.99 & $9.01 \mathrm{E}-07$ & 3.99 & $3.00 \mathrm{E}-06$ & 3.70 & $1.80 \mathrm{E}-06$ & 3.99 \\
\hline & 640 & $7.44 \mathrm{E}-07$ & 3.01 & $5.60 \mathrm{E}-08$ & 4.01 & $2.15 \mathrm{E}-03$ & 2.00 & $8.94 \mathrm{E}-06$ & 3.00 & $5.66 \mathrm{E}-08$ & 4.01 & $5.60 \mathrm{E}-08$ & 4.01 & $1.51 \mathrm{E}-07$ & 4.31 & $1.12 \mathrm{E}-07$ & 4.01 \\
\hline \multirow{5}{*}{$P^{3}$} & 20 & $3.18 \mathrm{E}-04$ & - & $7.32 \mathrm{E}-05$ & - & $4.31 \mathrm{E}-02$ & - & $3.34 \mathrm{E}-03$ & - & $1.88 \mathrm{E}-04$ & - & $7.28 \mathrm{E}-05$ & - & $2.16 \mathrm{E}-04$ & 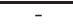 & $1.41 \mathrm{E}-04$ & - \\
\hline & 40 & $1.71 \mathrm{E}-05$ & 4.21 & $1.02 \mathrm{E}-06$ & 6.16 & $5.49 \mathrm{E}-03$ & 2.97 & $2.04 \mathrm{E}-04$ & 4.03 & $5.17 \mathrm{E}-06$ & 5.19 & $1.02 \mathrm{E}-06$ & 6.16 & $3.07 \mathrm{E}-06$ & 6.14 & $2.03 \mathrm{E}-06$ & 6.12 \\
\hline & 80 & $1.03 \mathrm{E}-06$ & 4.05 & $1.55 \mathrm{E}-08$ & 6.04 & $6.89 \mathrm{E}-04$ & 2.99 & $1.27 \mathrm{E}-05$ & 4.01 & $1.63 \mathrm{E}-07$ & 4.99 & $1.54 \mathrm{E}-08$ & 6.04 & $4.63 \mathrm{E}-08$ & 6.05 & $3.10 \mathrm{E}-08$ & 6.03 \\
\hline & 160 & $6.41 \mathrm{E}-08$ & 4.01 & $2.41 \mathrm{E}-10$ & 6.01 & $8.62 \mathrm{E}-05$ & 3.00 & $7.91 \mathrm{E}-07$ & 4.00 & $5.04 \mathrm{E}-09$ & 5.01 & $2.39 \mathrm{E}-10$ & 6.01 & $7.18 \mathrm{E}-10$ & 6.01 & $4.81 \mathrm{E}-10$ & 6.01 \\
\hline & 320 & $4.00 \mathrm{E}-09$ & 4.00 & 3.76E- 12 & 6.00 & $1.08 \mathrm{E}-05$ & 3.00 & $4.94 \mathrm{E}-08$ & 4.00 & $1.57 \mathrm{E}-10$ & 5.01 & 3.73E- 12 & 6.00 & $1.12 \mathrm{E}-11$ & 6.00 & $7.51 \mathrm{E}-12$ & 6.00 \\
\hline \multirow{5}{*}{$P^{4}$} & 10 & $5.04 \mathrm{E}-04$ & - & $7.75 \mathrm{E}-05$ & - & $1.14 \mathrm{E}-01$ & - & $1.32 \mathrm{E}-02$ & - & $2.21 \mathrm{E}-04$ & - & $7.63 \mathrm{E}-05$ & - & $2.15 \mathrm{E}-04$ & - & $1.27 \mathrm{E}-04$ & - \\
\hline & 20 & $2.10 \mathrm{E}-05$ & 4.58 & $4.91 \mathrm{E}-07$ & 7.30 & $1.11 \mathrm{E}-02$ & 3.36 & $6.17 \mathrm{E}-04$ & 4.42 & $5.70 \mathrm{E}-06$ & 5.28 & $4.52 \mathrm{E}-07$ & 7.40 & $1.26 \mathrm{E}-06$ & 7.42 & $8.66 \mathrm{E}-07$ & 7.20 \\
\hline & 40 & $7.32 \mathrm{E}-07$ & 4.84 & $2.65 \mathrm{E}-09$ & 7.53 & $8.01 \mathrm{E}-04$ & 3.79 & $2.17 \mathrm{E}-05$ & 4.83 & $1.05 \mathrm{E}-07$ & 5.76 & $2.05 \mathrm{E}-09$ & 7.78 & $6.17 \mathrm{E}-09$ & 7.67 & $4.04 \mathrm{E}-09$ & 7.74 \\
\hline & 80 & $2.36 \mathrm{E}-08$ & 4.96 & $1.60 \mathrm{E}-11$ & 7.37 & $5.21 \mathrm{E}-05$ & 3.94 & $6.76 \mathrm{E}-07$ & 5.01 & $1.72 \mathrm{E}-09$ & 5.93 & $8.31 \mathrm{E}-12$ & 7.94 & $2.57 \mathrm{E}-11$ & 7.91 & $1.66 \mathrm{E}-11$ & 7.93 \\
\hline & 160 & $7.42 \mathrm{E}-10$ & 4.99 & $1.13 \mathrm{E}-13$ & 7.15 & $3.29 \mathrm{E}-06$ & 3.99 & $2.19 \mathrm{E}-08$ & 4.95 & $2.72 \mathrm{E}-11$ & 5.98 & $3.27 \mathrm{E}-14$ & 7.99 & $9.96 \mathrm{E}-14$ & 8.01 & $6.55 \mathrm{E}-14$ & 7.98 \\
\hline
\end{tabular}

Table 7: Example 5.1 Error table for intermediate quantities when using central flux on uniform mesh. Ending time $T_{e}=1$, $x \in[0,2 \pi]$.

\begin{tabular}{|c|c|c|c|c|c|c|c|c|c|}
\hline & $\mathrm{N}$ & $\left\|\zeta_{h}\right\|$ error & order & $\left\|\left(\zeta_{h}\right)_{x x}\right\|$ & order & $E_{\left[\zeta_{h}\right]}$ & order & $E_{\left[\left(\zeta_{h}\right)_{x}\right]}$ & order \\
\hline \multirow{5}{*}{$P^{2}$} & 40 & $4.53 \mathrm{E}-03$ & - & $3.84 \mathrm{E}-02$ & - & $3.04 \mathrm{E}-05$ & - & $2.79 \mathrm{E}-04$ & - \\
& 80 & $3.15 \mathrm{E}-04$ & 3.85 & $3.03 \mathrm{E}-03$ & 3.66 & $1.16 \mathrm{E}-06$ & 4.71 & $8.31 \mathrm{E}-06$ & 5.07 \\
& 160 & $2.02 \mathrm{E}-05$ & 3.96 & $1.29 \mathrm{E}-04$ & 4.55 & $1.79 \mathrm{E}-07$ & 2.70 & $1.31 \mathrm{E}-06$ & 2.66 \\
& 320 & $1.27 \mathrm{E}-06$ & 3.99 & $1.59 \mathrm{E}-05$ & 3.03 & $7.14 \mathrm{E}-09$ & 4.65 & $5.08 \mathrm{E}-08$ & 4.69 \\
& 640 & $7.92 \mathrm{E}-08$ & 4.01 & $5.73 \mathrm{E}-07$ & 4.79 & $2.90 \mathrm{E}-10$ & 4.62 & $2.11 \mathrm{E}-09$ & 4.59 \\
\hline \multirow{5}{*}{$P^{3}$} & 20 & $1.03 \mathrm{E}-04$ & - & $9.27 \mathrm{E}-04$ & - & $4.27 \mathrm{E}-08$ & - & $5.25 \mathrm{E}-06$ & - \\
& 40 & $1.44 \mathrm{E}-06$ & 6.16 & $1.29 \mathrm{E}-05$ & 6.17 & $1.75 \mathrm{E}-10$ & 7.93 & $4.32 \mathrm{E}-08$ & 6.93 \\
& 80 & $2.18 \mathrm{E}-08$ & 6.04 & $1.99 \mathrm{E}-07$ & 6.02 & $4.23 \mathrm{E}-13$ & 8.69 & $1.64 \mathrm{E}-10$ & 8.04 \\
& 160 & $3.38 \mathrm{E}-10$ & 6.01 & $3.05 \mathrm{E}-09$ & 6.03 & $2.91 \mathrm{E}-16$ & 10.50 & $3.24 \mathrm{E}-14$ & 12.31 \\
& 320 & $5.28 \mathrm{E}-12$ & 6.00 & $4.76 \mathrm{E}-11$ & 6.00 & $1.28 \mathrm{E}-18$ & 7.83 & $1.41 \mathrm{E}-15$ & 4.52 \\
\hline \multirow{5}{*}{$P^{4}$} & 10 & $1.06 \mathrm{E}-04$ & - & $1.05 \mathrm{E}-03$ & - & $7.67 \mathrm{E}-07$ & - & $2.26 \mathrm{E}-05$ & - \\
& 20 & $6.37 \mathrm{E}-07$ & 7.37 & $7.12 \mathrm{E}-06$ & 7.21 & $3.03 \mathrm{E}-09$ & 7.99 & $7.70 \mathrm{E}-08$ & 8.20 \\
& 40 & $2.88 \mathrm{E}-09$ & 7.79 & $3.20 \mathrm{E}-08$ & 7.80 & $5.78 \mathrm{E}-12$ & 9.03 & $6.36 \mathrm{E}-11$ & 10.24 \\
& 80 & $1.17 \mathrm{E}-11$ & 7.94 & $1.84 \mathrm{E}-10$ & 7.44 & $9.33 \mathrm{E}-15$ & 9.28 & $5.24 \mathrm{E}-13$ & 6.92 \\
& 160 & $4.63 \mathrm{E}-14$ & 7.98 & $2.45 \mathrm{E}-12$ & 6.23 & $3.04 \mathrm{E}-17$ & 8.26 & $8.82 \mathrm{E}-16$ & 9.21 \\
\hline
\end{tabular}


Table 8: Example 5.1 Error table when using flux parameters: $\alpha_{1}=0.25, \beta_{1}=\frac{2}{h}, \frac{5}{h}, \frac{9}{h}, \beta_{2}=0$ on uniform mesh. Ending time $T_{e}=1, x \in[0,2 \pi]$.

\begin{tabular}{|c|c|c|c|c|c|c|c|c|c|c|c|c|c|c|c|c|c|}
\hline & $\mathrm{N}$ & $L^{2}$ error & order & $E_{P}$ & order & $E_{u_{x x}}$ & order & $E_{u_{x}}$ & order & $E_{u}$ & order & $E_{f}$ & order & $E_{f_{x}}$ & order & $E_{c}$ & order \\
\hline \multirow{5}{*}{$P^{2}$} & 80 & $1.41 \mathrm{E}-03$ & 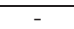 & $8.17 \mathrm{E}-05$ & - & DNE & - & $1.15 \mathrm{E}-02$ & - & $1.19 \mathrm{E}-04$ & - & $8.07 \mathrm{E}-05$ & 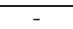 & $1.71 \mathrm{E}-04$ & - & $1.61 \mathrm{E}-04$ & 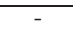 \\
\hline & 160 & $1.65 \mathrm{E}-04$ & 3.09 & $4.74 \mathrm{E}-06$ & 4.11 & DNE & - & $1.34 \mathrm{E}-03$ & 3.11 & $6.89 \mathrm{E}-06$ & 4.11 & $4.67 \mathrm{E}-06$ & 4.11 & $5.39 \mathrm{E}-06$ & 4.99 & $9.34 \mathrm{E}-06$ & 4.11 \\
\hline & 320 & $2.03 \mathrm{E}-05$ & 3.02 & $2.92 \mathrm{E}-07$ & 4.02 & DNE & - & $1.65 \mathrm{E}-04$ & 3.02 & $4.21 \mathrm{E}-07$ & 4.03 & $2.86 \mathrm{E}-07$ & 4.03 & $1.82 \mathrm{E}-06$ & 1.57 & $5.75 \mathrm{E}-07$ & 4.02 \\
\hline & 640 & $2.53 \mathrm{E}-06$ & 3.01 & $1.80 \mathrm{E}-08$ & 4.03 & DNE & - & $2.05 \mathrm{E}-05$ & 3.01 & $2.62 \mathrm{E}-08$ & 4.01 & $1.78 \mathrm{E}-08$ & 4.00 & $1.40 \mathrm{E}-07$ & 3.70 & $3.58 \mathrm{E}-08$ & 4.01 \\
\hline & 1280 & $3.16 \mathrm{E}-07$ & 3.00 & $1.22 \mathrm{E}-09$ & 3.88 & DNE & - & $2.55 \mathrm{E}-06$ & 3.01 & $1.71 \mathrm{E}-09$ & 3.94 & $1.21 \mathrm{E}-09$ & 3.88 & $5.55 \mathrm{E}-09$ & 4.65 & $2.43 \mathrm{E}-09$ & 3.88 \\
\hline \multirow{5}{*}{$P^{3}$} & 20 & $8.27 \mathrm{E}-04$ & - & $4.58 \mathrm{E}-05$ & - & $2.98 \mathrm{E}-01$ & - & $6.63 \mathrm{E}-03$ & - & $3.40 \mathrm{E}-04$ & - & $3.50 \mathrm{E}-05$ & 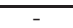 & $9.99 \mathrm{E}-05$ & - & $7.80 \mathrm{E}-05$ & - \\
\hline & 40 & $3.92 \mathrm{E}-05$ & 4.40 & $5.20 \mathrm{E}-07$ & 6.46 & $3.11 \mathrm{E}-02$ & 3.26 & $3.60 \mathrm{E}-04$ & 4.20 & $8.78 \mathrm{E}-06$ & 5.27 & $4.26 \mathrm{E}-07$ & 6.36 & $1.24 \mathrm{E}-06$ & 6.34 & $9.58 \mathrm{E}-07$ & 6.35 \\
\hline & 80 & $2.29 \mathrm{E}-06$ & 4.10 & 7.54E-09 & 6.11 & $3.72 \mathrm{E}-03$ & 3.06 & $2.18 \mathrm{E}-05$ & 4.05 & $2.61 \mathrm{E}-07$ & 5.07 & $6.24 \mathrm{E}-09$ & 6.09 & $1.87 \mathrm{E}-08$ & 6.05 & $1.42 \mathrm{E}-08$ & 6.08 \\
\hline & 160 & $1.40 \mathrm{E}-07$ & 4.03 & $1.16 \mathrm{E}-10$ & 6.03 & $4.60 \mathrm{E}-04$ & 3.02 & $1.35 \mathrm{E}-06$ & 4.01 & $8.05 \mathrm{E}-09$ & 5.02 & $9.60 \mathrm{E}-11$ & 6.02 & $2.86 \mathrm{E}-10$ & 6.03 & $2.19 \mathrm{E}-10$ & 6.02 \\
\hline & 320 & $8.74 \mathrm{E}-09$ & 4.01 & $1.80 \mathrm{E}-12$ & 6.01 & $5.74 \mathrm{E}-05$ & 3.00 & $8.43 \mathrm{E}-08$ & 4.00 & $2.50 \mathrm{E}-10$ & 5.01 & $1.49 \mathrm{E}-12$ & 6.01 & $4.47 \mathrm{E}-12$ & 6.00 & $3.41 \mathrm{E}-12$ & 6.00 \\
\hline \multirow{4}{*}{$P^{4}$} & 20 & $5.10 \mathrm{E}-04$ & - & $2.08 \mathrm{E}-04$ & - & $3.76 \mathrm{E}-01$ & - & $3.96 \mathrm{E}-03$ & - & $1.36 \mathrm{E}-04$ & - & $1.08 \mathrm{E}-05$ & - & $3.52 \mathrm{E}-05$ & - & $2.10 \mathrm{E}-05$ & - \\
\hline & 40 & $8.28 \mathrm{E}-06$ & 5.95 & $2.38 \mathrm{E}-07$ & 9.77 & $1.24 \mathrm{E}-02$ & 4.92 & $6.76 \mathrm{E}-05$ & 5.87 & $1.16 \mathrm{E}-06$ & 6.87 & $2.19 \mathrm{E}-08$ & 8.95 & $6.74 \mathrm{E}-08$ & 9.03 & $4.34 \mathrm{E}-08$ & 8.92 \\
\hline & 80 & $1.87 \mathrm{E}-07$ & 5.47 & $1.04 \mathrm{E}-09$ & 7.84 & $5.64 \mathrm{E}-04$ & 4.47 & $1.55 \mathrm{E}-06$ & 5.45 & $1.33 \mathrm{E}-08$ & 6.45 & $6.23 \mathrm{E}-11$ & 8.46 & $2.15 \mathrm{E}-10$ & 8.29 & $1.25 \mathrm{E}-10$ & 8.45 \\
\hline & 160 & $5.44 \mathrm{E}-09$ & 5.10 & $7.11 \mathrm{E}-12$ & 7.19 & $3.29 \mathrm{E}-05$ & 4.10 & $4.53 \mathrm{E}-08$ & 5.09 & $1.94 \mathrm{E}-10$ & 6.09 & $2.28 \mathrm{E}-13$ & 8.09 & $6.15 \mathrm{E}-13$ & 8.45 & $4.56 \mathrm{E}-13$ & 8.09 \\
\hline
\end{tabular}

Table 9: Example 5.1 Postprocessing error table for the four sets of parameters. Ending time $T_{e}=1$, uniform mesh on $x \in[0,2 \pi]$. The first row below labels the parameters by $\left(\tilde{\alpha_{1}}, \tilde{\beta_{1}}, \tilde{\beta_{2}}\right)$.

\begin{tabular}{|c|c|c|c|c|c|c|c|c|c|}
\hline \multicolumn{2}{|c|}{ Fluxes } & \multicolumn{2}{|c|}{$(0.5,0,0)$} & \multicolumn{2}{|c|}{$(0,0,0)$} & \multicolumn{2}{|c|}{$(0.3,0.4,0.4)$} & \multicolumn{2}{|c|}{$(0.25,\{2,5,9\}, 0)$} \\
\hline & $\mathrm{N}$ & $E^{*}$ & order & $E^{*}$ & order & $E^{*}$ & order & $E^{*}$ & order \\
\hline \multirow{5}{*}{$P^{2}$} & 10 & $1.00 \mathrm{E}+00$ & & $2.81 \mathrm{E}-01$ & 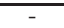 & $1.00 \mathrm{E}+00$ & & $1.53 \mathrm{E}-01$ & \\
\hline & 20 & $2.84 \mathrm{E}-01$ & 1.81 & $3.71 \mathrm{E}-02$ & 2.92 & $1.20 \mathrm{E}-01$ & 3.06 & $8.05 \mathrm{E}-02$ & 0.93 \\
\hline & 40 & $2.11 \mathrm{E}-02$ & 3.75 & $3.23 \mathrm{E}-03$ & 3.52 & $9.63 \mathrm{E}-03$ & 3.64 & $2.68 \mathrm{E}-03$ & 4.91 \\
\hline & 80 & $1.37 \mathrm{E}-03$ & 3.94 & $2.24 \mathrm{E}-04$ & 3.85 & $7.55 \mathrm{E}-04$ & 3.67 & $1.20 \mathrm{E}-04$ & 4.49 \\
\hline & 160 & $8.69 \mathrm{E}-05$ & 3.98 & $1.44 \mathrm{E}-05$ & 3.96 & $5.13 \mathrm{E}-05$ & 3.88 & $6.99 \mathrm{E}-06$ & 4.10 \\
\hline \multirow{5}{*}{$P^{3}$} & 10 & $1.00 \mathrm{E}+00$ & - & $1.00 \mathrm{E}+00$ & - & $1.00 \mathrm{E}+00$ & - & $1.00 \mathrm{E}+00$ & - \\
\hline & 20 & $6.04 \mathrm{E}-02$ & 4.05 & $6.29 \mathrm{E}-02$ & 3.99 & $6.05 \mathrm{E}-02$ & 4.05 & $7.02 \mathrm{E}-02$ & 3.83 \\
\hline & 40 & $5.39 \mathrm{E}-04$ & 6.81 & $6.05 \mathrm{E}-04$ & 6.70 & $5.26 \mathrm{E}-04$ & 6.85 & $5.46 \mathrm{E}-04$ & 7.01 \\
\hline & 80 & $3.28 \mathrm{E}-06$ & 7.36 & $5.04 \mathrm{E}-06$ & 6.91 & $2.82 \mathrm{E}-06$ & 7.54 & $2.91 \mathrm{E}-06$ & 7.55 \\
\hline & 160 & $3.14 \mathrm{E}-08$ & 6.70 & $6.49 \mathrm{E}-08$ & 6.28 & $2.04 \mathrm{E}-08$ & 7.11 & $1.79 \mathrm{E}-08$ & 7.34 \\
\hline \multirow{5}{*}{$P^{4}$} & 10 & $1.00 \mathrm{E}+00$ & 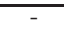 & $1.00 \mathrm{E}+00$ & 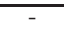 & $1.00 \mathrm{E}+00$ & 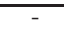 & $1.00 \mathrm{E}+00$ & - \\
\hline & 20 & $4.54 \mathrm{E}-02$ & 4.46 & $4.54 \mathrm{E}-02$ & 4.46 & $4.54 \mathrm{E}-02$ & 4.46 & $4.54 \mathrm{E}-02$ & 4.46 \\
\hline & 40 & $1.32 \mathrm{E}-04$ & 8.42 & $1.32 \mathrm{E}-04$ & 8.42 & $1.32 \mathrm{E}-04$ & 8.42 & $1.36 \mathrm{E}-04$ & 8.39 \\
\hline & 80 & $1.70 \mathrm{E}-07$ & 9.60 & $1.70 \mathrm{E}-07$ & 9.60 & $1.70 \mathrm{E}-07$ & 9.60 & $1.66 \mathrm{E}-07$ & 9.67 \\
\hline & 160 & $1.79 \mathrm{E}-10$ & 9.89 & $1.80 \mathrm{E}-10$ & 9.89 & $1.79 \mathrm{E}-10$ & 9.89 & $1.75 \mathrm{E}-10$ & 9.89 \\
\hline
\end{tabular}

\section{Conclusions and Future Work}

In this paper, we studied the superconvergence property of the UWDG methods with scale invariant fluxes for linear Schrödinger equation in one dimension with periodic boundary condition. When $k$ is odd, and $k$ is even with the flux parameters satisfying certain assumptions, we proved $(2 k)$-th order convergence rate for cell averages and numerical flux, and $(2 k-1)$-th order convergence rate for numerical flux for the derivative. In addition, the numerical solution is convergent towards a special projection with $(k+\min (3, k))$-th order convergence rate. The results were obtained by the correction function techniques in [8] and intermediate results of the superconvergence of the second derivative and jump across cell interfaces of the difference between numerical solution and projected exact solution. However, for some special flux parameter choices when $k$ is even, such intermediate results are no longer valid. Therefore, under this condition, the provable convergence rate is one order lower than the previous cases, though numerical results seem to suggest otherwise. Indeed, our numerical experiments show $(2 k)$-th convergence rate for cell average, numerical flux, and the numerical flux for the derivative, and $(k+\min (3, k))$-th convergence rate of the difference between numerical solution and a special projection. The surprising finding that the numerical flux of solution and derivative of solution both converge at rate $2 k$ indicates that our proof can be improved for the numerical flux for derivative. We also showed that the convergence order of UWDG scheme can be enhanced to $2 k$ by postprocessing. In numerical tests, the orders of convergence for the postprocessed solution are at least $2 k$, and can go up to $2 k+2$ when $k \geq 3$.

There are some recent development of extending the correction function technique to nonlinear equations and high dimensional equations. It would be our future work to extend 
this work to nonlinear Schrödinger equations in two dimensional setting. Also, there are developments in the negative norm estimates for the error of DG schemes for nonlinear equations, it will also be an interesting subject to consider.

\section{References}

[1] S. Adjerid, K. D. Devine, J. E. Flaherty, and L. Krivodonova. A posteriori error estimation for discontinuous Galerkin solutions of hyperbolic problems. Computer methods in applied mechanics and engineering, 191(11-12):1097-1112, 2002.

[2] S. Adjerid and T. C. Massey. Superconvergence of discontinuous Galerkin solutions for a nonlinear scalar hyperbolic problem. Computer methods in applied mechanics and engineering, 195(25-28):3331-3346, 2006.

[3] J. Bona, H. Chen, O. Karakashian, and Y. Xing. Conservative, discontinuous Galerkinmethods for the generalized Korteweg-de Vries equation. Mathematics of Computation, 82(283):1401-1432, 2013.

[4] J. H. Bramble and A. H. Schatz. Higher order local accuracy by averaging in the finite element method. Mathematics of Computation, 31(137):94-111, 1977.

[5] W. Cao, D. Li, Y. Yang, and Z. Zhang. Superconvergence of discontinuous Galerkin methods based on upwind-biased fluxes for 1d linear hyperbolic equations. ESAIM: Mathematical Modelling and Numerical Analysis, 51(2):467-486, 2017.

[6] W. Cao, H. Liu, and Z. Zhang. Superconvergence of the direct discontinuous Galerkin method for convection-diffusion equations. Numerical Methods for Partial Differential Equations, 33(1):290-317, 2017.

[7] W. Cao, C.-W. Shu, Y. Yang, and Z. Zhang. Superconvergence of discontinuous Galerkin method for scalar nonlinear hyperbolic equations. SIAM Journal on Numerical Analysis, $56(2): 732-765,2018$.

[8] W. Cao, Z. Zhang, and Q. Zou. Superconvergence of discontinuous Galerkin methods for linear hyperbolic equations. SIAM Journal on Numerical Analysis, 52(5):2555-2573, 2014.

[9] O. Cessenat and B. Despres. Application of an ultra weak variational formulation of elliptic PDEs to the two-dimensional helmholtz problem. SIAM journal on numerical analysis, 35(1):255-299, 1998.

[10] A. Chen, F. Li, and Y. Cheng. An ultra-weak discontinuous Galerkin method for Schrödingerequation in one dimension. Journal of Scientific Computing, 78(2):772-815, Feb 2019.

[11] Y. Cheng and C.-W. Shu. A discontinuous Galerkin finite element method for time dependent partial differential equations with higher order derivatives. Mathematics of Computation, 77(262):699-730, 2008. 
[12] Y. Cheng and C.-W. Shu. Superconvergence of discontinuous Galerkin and local discontinuous Galerkin schemes for linear hyperbolic and convection-diffusion equations in one space dimension. SIAM Journal on Numerical Analysis, 47(6):4044-4072, 2010.

[13] B. Cockburn, M. Luskin, C.-W. Shu, and E. Süli. Enhanced accuracy by post-processing for finite element methods for hyperbolic equations. Mathematics of Computation, $72(242): 577-606,2003$.

[14] B. Cockburn and C.-W. Shu. Runge-kutta discontinuous Galerkin methods for convection-dominated problems. Journal of scientific computing, 16(3):173-261, 2001.

[15] P. J. Davis. Circulant matrices. Wiley, New York, 1979.

[16] L. Ji, Y. Xu, and J. K. Ryan. Negative-order norm estimates for nonlinear hyperbolic conservation laws. Journal of Scientific Computing, 54(2):531-548, Feb 2013.

[17] X. Liang, A. Q. M. Khaliq, and Y. Xing. Fourth order exponential time differencing method with local discontinuous Galerkin approximation for coupled nonlinear Schrödinger equations. Communications in Computational Physics, 17(2):510-541, 2015.

[18] W. Lu, Y. Huang, and H. Liu. Mass preserving discontinuous Galerkin methods for Schrödinger equations. Journal of Computational Physics, 282:210-226, 2015.

[19] X. Meng and J. K. Ryan. Discontinuous Galerkin methods for nonlinear scalar hyperbolic conservation laws: divided difference estimates and accuracy enhancement. Numerische Mathematik, 136(1):27-73, May 2017.

[20] M. S. Mock and P. D. Lax. The computation of discontinuous solutions of linear hyperbolic equations. Communications on Pure and Applied Mathematics, 31(4):423-430, 1978.

[21] W. H. Reed and T. Hill. Triangular mesh methods for the neutron transport equation. Technical report, Los Alamos Scientific Lab., N. Mex.(USA), 1973.

[22] J. Ryan, C.-W. Shu, and H. Atkins. Extension of a post processing technique for the discontinuous Galerkin method for hyperbolic equations with application to an aeroacoustic problem. SIAM Journal on Scientific Computing, 26(3):821-843, 2005.

[23] J. Shen, T. Tang, and L.-L. Wang. Spectral methods: algorithms, analysis and applications, volume 41. Springer Science \& Business Media, 2011.

[24] C.-W. Shu. Discontinuous Galerkin methods for time-dependent convection dominated problems: Basics, recent developments and comparison with other methods. In A. C. G.R. Barrenechea, F. Brezzi and E. Georgoulis, editors, Building Bridges: Connections and Challenges in Modern Approaches to Numerical Partial Differential Equations, volume 114, pages 369-397. Springer, Switzerland, 2016.

[25] Y. Xu and C.-W. Shu. Local discontinuous Galerkin methods for nonlinear Schrödinger equations. Journal of Computational Physics, 205(1):72-97, 2005. 
[26] Y. Xu and C.-W. Shu. Optimal error estimates of the semidiscrete local discontinuous Galerkin methods for high order wave equations. SIAM Journal on Numerical Analysis, 50(1):79-104, 2012.

[27] Y. Yang and C.-W. Shu. Analysis of optimal superconvergence of discontinuous Galerkin method for linear hyperbolic equations. SIAM Journal on Numerical Analysis, 50(6):3110-3133, 2012.

[28] L. Zhou, Y. Xu, Z. Zhang, and W. Cao. Superconvergence of local discontinuous Galerkin method for one-dimensional linear schrödinger equations. Journal of Scientific Computing, 73(2-3):1290-1315, 2017.

\section{A Appendix}

\section{A.1 Collections of intermediate results}

In this section, we list some results that will be used in the rest of the appendix. First, we gather some results from [10]. (59) - (63) in [10] yields

$$
\sum_{j=1}^{N}\left\|r_{j}\right\|_{\infty} \leq C, \quad \text { if } \mathrm{A} 2
$$

where $r_{j}$ has been defined in (17). (59) and (71) and the first equation in A.3.3 in [10] yields

$$
\sum_{j=1}^{N}\left\|r_{j}\right\|_{\infty} \leq C h^{-2}, \quad \text { if A3. }
$$

Next, we provide estimates of the Legendre coefficients in neighboring cells of equal size.

If $u \in W^{k+2+n, \infty}(I)$, then expand $\hat{u}_{j}(\xi)$ at $\xi=-1$ in (9) by Taylor series, we have for $m \geq k+1, \exists z \in[-1,1]$, s.t.

$$
\begin{aligned}
u_{j, m} & =C \int_{-1}^{1} \frac{d}{d \xi^{k+1}}\left(\sum_{s=0}^{n} \frac{d}{d \xi^{s}} \hat{u}_{j}(-1) \frac{(\xi+1)^{s}}{s !}+\frac{d}{d \xi^{n+1}} \hat{u}_{j}(z) \frac{(\xi+1)^{n+1}}{(n+1) !}\right) \frac{d}{d \xi^{m-k-1}}\left(\xi^{2}-1\right)^{m} d \xi \\
& =\sum_{s=0}^{n} \theta_{s} h_{j}^{k+1+s} u^{(k+1+s)}\left(x_{j-\frac{1}{2}}\right)+O\left(h_{j}^{k+2+n}|u|_{W^{k+2+n, \infty}\left(I_{j}\right)}\right),
\end{aligned}
$$

where $\theta_{l}$ are constants independent of $u$ and $h_{j}$.

Therefore, when $h_{j}=h_{j+1}$, we use Taylor expansion again, and compute the difference of two $u_{j, m}$ from neighboring cells

$$
u_{j, m}-u_{j+1, m}=\sum_{s=1}^{n} \mu_{s} h_{j}^{k+1+s} u^{(k+1+s)}\left(x_{j-\frac{1}{2}}\right)+O\left(h_{j}^{k+2+n}|u|_{W^{k+2+n, \infty}\left(I_{j} \cup I_{j+1}\right)}\right) .
$$

Then we obtain the estimates

$$
\left|u_{j, m}-u_{j+1, m}+\sum_{s=1}^{n} \mu_{s} h_{j}^{k+1+s} u^{(k+1+s)}\left(x_{j-\frac{1}{2}}\right)\right| \leq C h^{k+2+n}|u|_{W^{k+2+n, \infty}\left(I_{j} \cup I_{j+1}\right)},
$$

where $\mu_{s}$ are constants independent of $u$ and $h_{j}$. 


\section{A.1.1 Two convolution-like operators}

In the proof of Lemmas 3.8 and 3.9 in [10], we used Fourier analysis for error analysis. Now we extract the main ideas and generalize the results to facilitate the proof of superconvergence results in Lemmas 3.4 and 4.2 .

We define two operators on a periodic functions $u$ in $L^{2}(I)$ :

$$
\begin{aligned}
\bigotimes_{\lambda} u(x) & =\frac{1}{1-\lambda^{N}} \sum_{l=0}^{N-1} \lambda^{l} u\left(x+L \frac{l}{N}\right), \\
\boxplus u(x) & =\sum_{l=0}^{N-1}(-1)^{l} \frac{-N+2 l}{2} u\left(x+L \frac{l}{N}\right),
\end{aligned}
$$

where $L=b-a$ is the size of $I$.

Expand $u$ by Fourier series, i.e., $u(x)=\sum_{n=-\infty}^{\infty} \hat{f}(n) e^{2 \pi i n x / L}$, we have

$$
\begin{aligned}
\bigotimes_{\lambda} u(x) & =\frac{1}{1-\lambda^{N}} \sum_{l=0}^{N-1} \lambda^{l} \sum_{n=-\infty}^{\infty} \hat{f}(n) e^{i n\left(\frac{2 \pi}{L} x+2 \pi \frac{l}{N}\right)}=\frac{1}{1-\lambda^{N}} \sum_{n=-\infty}^{\infty} \hat{f}(n) e^{\frac{2 \pi}{L} i n x} \sum_{l=0}^{N-1}\left(\lambda e^{\left.i 2 \pi \frac{n}{N}\right)^{l}}\right. \\
& =\sum_{n=-\infty}^{\infty} \frac{\hat{f}(n)}{1-\lambda e^{2 \pi i \frac{n}{N}}} e^{\frac{2 \pi}{L} i n x}, \\
\boxplus u(x) & =\sum_{l=0}^{N-1}(-1)^{l} \frac{-N+2 l}{2} \sum_{n=-\infty}^{\infty} \hat{f}(n) e^{i n\left(\frac{2 \pi}{L} x+2 \pi \frac{l}{N}\right)}=\sum_{n=-\infty}^{\infty} \hat{f}(n) e^{\frac{2 \pi}{L} i n x} \sum_{l=0}^{N-1} \frac{-N+2 l}{2}\left(-e^{\left.i 2 \pi \frac{n}{N}\right)^{l}}\right. \\
& =\sum_{n=-\infty}^{\infty} \frac{-2 e^{2 \pi i \frac{n}{N}}}{\left(1+e^{2 \pi i \frac{n}{N}}\right)^{2}} \hat{f}(n) e^{\frac{2 \pi}{L} i n x} .
\end{aligned}
$$

In addition, we can apply the operator on the same function recursively, we have

$$
\begin{aligned}
\otimes_{\lambda_{1}}^{\nu_{1}} \cdots \bigotimes_{\lambda_{n}}^{\nu_{n}} u(x) & =\sum_{n=-\infty}^{\infty} \frac{1}{\left(1-\lambda_{1} e^{2 \pi i \frac{n}{N}}\right)^{\nu_{1}}} \cdots \frac{1}{\left(1-\lambda_{n} e^{2 \pi i \frac{n}{N}}\right)^{\nu_{n}}} \hat{f}(n) e^{i \frac{2 \pi}{L} i n x}, \\
\left(\bigotimes_{\lambda}\right)^{\nu} u(x) & =\sum_{n=-\infty}^{\infty} \frac{\hat{f}(n)}{\left(1-\lambda e^{2 \pi i \frac{n}{N}}\right)^{\nu}} e^{\frac{2 \pi}{L} i n x}, \\
(\boxplus)^{\nu} u(x) & =\sum_{n=-\infty}^{\infty}\left(\frac{-2 e^{2 \pi i \frac{n}{N}}}{\left(1+e^{2 \pi i \frac{n}{N}}\right)^{2}}\right)^{\nu} \hat{f}(n) e^{\frac{2 \pi}{L} i n x} .
\end{aligned}
$$

As shown in the proof of Lemmas 3.8 and 3.9 in [10], if $\lambda_{i}, i \leq n$ is a complex number with $\left|\lambda_{i}\right|=1$, independent of $h$, then

$$
\bigotimes_{\lambda_{1}}^{\nu_{1}} \cdots \bigotimes_{\lambda_{n}}^{\nu_{n}} u(x) \leq C|u|_{W^{1+\sum_{i=1}^{n} \nu_{i}, 1}(I)}, \quad(\boxplus u(x))^{\nu} \leq C|u|_{W^{1+2 \nu, 1}(I)} .
$$

\section{A.2 Proof of Lemma 3.1}

Proof.

$$
A_{j}+B_{j}=G\left[L_{j, k-1}^{-}, L_{j, k}^{-}\right]+H\left[L_{j, k-1}^{+}, L_{j, k}^{+}\right]=\frac{1}{2}\left[\begin{array}{cc}
1 & 0 \\
0 & \frac{1}{h_{j}}
\end{array}\right] M_{+}+\left[\begin{array}{cc}
\alpha_{1} & -\beta_{2} \\
-\beta_{1} & -\alpha_{1}
\end{array}\right]\left[\begin{array}{cc}
1 & 0 \\
0 & \frac{1}{h_{j}}
\end{array}\right] M_{-},
$$


where

$$
M_{ \pm}=\left[\begin{array}{cc}
1 & 0 \\
0 & h_{j}
\end{array}\right]\left[L_{j, k-1}^{-} \pm L_{j, k-1}^{+}, L_{j, k}^{-} \pm L_{j, k}^{+}\right]=\left[\begin{array}{cc}
1 \pm(-1)^{k-1} & 1 \pm(-1)^{k} \\
k(k-1)\left(1 \pm(-1)^{k}\right) & k(k+1)\left(1 \pm(-1)^{k+1}\right)
\end{array}\right] .
$$

Therefore,

$$
\left(A_{j}+B_{j}\right)^{-1}=\frac{1}{D_{1}} M_{-}^{-1}\left[\begin{array}{cc}
-\alpha_{1} & \beta_{2}-\frac{h_{j}}{2 k\left(k+(-1)^{k}\right)} \\
\beta_{1} h_{j}-\frac{k\left(k-(-1)^{k}\right)}{2} & \alpha_{1} h_{j}
\end{array}\right]
$$

where $D_{1}=\frac{(-1)^{k} h_{j}}{2 k\left(k+(-1)^{k}\right)}\left((-1)^{k} \Gamma_{j}+\Lambda_{j}\right)$ is bounded by definitions of $\Gamma_{j}, \Lambda_{j}$ and mesh regularity condition. Then

$$
\begin{aligned}
& \left(A_{j}+B_{j}\right)^{-1} G\left[\begin{array}{cc}
1 & 0 \\
0 & \frac{1}{h_{j}}
\end{array}\right]=\frac{1}{D_{1}} M_{-}^{-1}\left[\begin{array}{cc}
\tilde{\beta}_{2} h h_{j}^{-1}-\frac{1}{2 k\left(k+(-1)^{k}\right)} \\
\tilde{\beta}_{1} h^{-1} h_{j}-\frac{k\left(k-(-1)^{k}\right)}{2} & \alpha_{1}
\end{array}\right]\left[\begin{array}{cc}
\frac{1}{2}+\alpha_{1} & -\tilde{\beta}_{2} h h_{j}^{-1} \\
-\tilde{\beta}_{1} h^{-1} h_{j} & \frac{1}{2}-\alpha_{1}
\end{array}\right], \\
& \left(A_{j}+B_{j}\right)^{-1} H\left[\begin{array}{cc}
1 & 0 \\
0 & \frac{1}{h_{j}}
\end{array}\right]=\frac{1}{D_{1}} M_{-}^{-1}\left[\begin{array}{ccc}
-\alpha_{1} & \tilde{\beta}_{2} h h_{j}^{-1}-\frac{1}{2 k\left(k+(-1)^{k}\right)} \\
\tilde{\beta}_{1} h^{-1} h_{j}-\frac{k\left(k-(-1)^{k}\right)}{2} & \alpha_{1}
\end{array}\right]\left[\begin{array}{cc}
\frac{1}{2}-\alpha_{1} & \tilde{\beta}_{2} h h_{j}^{-1} \\
\tilde{\beta}_{1} h^{-1} h_{j} & \frac{1}{2}+\alpha_{1}
\end{array}\right]
\end{aligned}
$$

and

$$
\mathcal{M}_{j, m}=\left(A_{j}+B_{j}\right)^{-1} G\left[\begin{array}{cc}
1 & 0 \\
0 & \frac{1}{h_{j}}
\end{array}\right]\left[\begin{array}{c}
1 \\
m(m+1)
\end{array}\right]+(-1)^{m}\left(A_{j}+B_{j}\right)^{-1} H\left[\begin{array}{cc}
1 & 0 \\
0 & \frac{1}{h_{j}}
\end{array}\right]\left[\begin{array}{c}
1 \\
-m(m+1)
\end{array}\right] .
$$

By mesh regularity condition, $\exists \sigma_{1}, \sigma_{2}$, s.t., $\sigma_{1} h_{j} \leq h \leq \sigma_{2} h_{j}$ and the proof is complete.

\section{A.3 Proof of Lemma 3.2}

By Definition 3.1, $\left.P_{h}^{\star} u\right|_{I_{j}}=\sum_{m=0}^{k-2} u_{j, m} L_{j, m}+\dot{u}_{j, k-1} L_{j, k-1}+\dot{u}_{j, k} L_{j, k}$. We solve the two coefficients $\dot{u}_{j, k-1}, \dot{u}_{j, k}$ on every cell $I_{j}$ according to definition (14).

If assumption A1 is satisfied, it has been shown in Lemma 3.1 in [10] that (14) is equivalent to (21). Substitute $u$ and $u_{x}$ by (8), we obtain the following equation

$$
\left(A_{j}+B_{j}\right)\left[\begin{array}{c}
\dot{u}_{j, k-1} \\
\hat{u}_{j, k}
\end{array}\right]=\left(A_{j}+B_{j}\right)\left[\begin{array}{c}
u_{j, k-1} \\
u_{j, k}
\end{array}\right]+\sum_{m=k+1}^{\infty} u_{j, m}\left(G L_{j, m}^{-}+H L_{j, m}^{+}\right),
$$

the existence and uniqueness of the system above is ensured by assumption A1, that is, $\operatorname{det}\left(A_{j}+B_{j}\right)=2(-1)^{k} \Gamma_{j} \neq 0$. Thus, (22) is proven.

If any of the assumptions A2/A3 is satisfied, we obtain

$$
A\left[\begin{array}{c}
\dot{u}_{j, k-1} \\
\dot{u}_{j, k}
\end{array}\right]+B\left[\begin{array}{c}
\dot{u}_{j+1, k-1} \\
\dot{u}_{j+1, k}
\end{array}\right]=\sum_{m=k-1}^{\infty} u_{j, m} G L_{m}^{-}+u_{j+1, m} H L_{m}^{+},
$$


which can be solved by a global linear system with coefficient matrix $M$. The solution is

$$
\begin{aligned}
{\left[\begin{array}{c}
\dot{u}_{j, k-1} \\
\dot{u}_{j, k}
\end{array}\right] } & =\sum_{l=0}^{N-1} r_{l} A^{-1}\left(A\left[\begin{array}{c}
u_{j+l, k-1} \\
u_{j+l, k}
\end{array}\right]+B\left[\begin{array}{c}
u_{j+l+1, k-1} \\
u_{j+l+1, k}
\end{array}\right]+\sum_{m=k+1}^{\infty} u_{j+l, m} G L_{m}^{-}+u_{j+l+1, m} H L_{m}^{+}\right) \\
& =\sum_{l=0}^{N-1} r_{l}\left(\left[\begin{array}{c}
u_{j+l, k-1} \\
u_{j+l, k}
\end{array}\right]-Q\left[\begin{array}{c}
u_{j+l+1, k-1} \\
u_{j+l+1, k}
\end{array}\right]+\sum_{m=k+1}^{\infty} u_{j+l, m}\left[L_{k-1}^{-}, L_{k}^{-}\right]^{-1} L_{m}^{-}-u_{j+l+1, m} Q\left[L_{k-1}^{+}, L_{k}^{+}\right]^{-1} L_{m}^{+}\right) \\
& =\left[\begin{array}{c}
u_{j, k-1} \\
u_{j, k}
\end{array}\right]+\sum_{m=k+1}^{\infty}\left(\sum_{l=1}^{N-1} u_{j+l, m} V_{2, m}+u_{j, m} r_{0}\left[L_{k-1}^{-}, L_{k}^{-}\right]^{-1} L_{m}^{-}-u_{j+N, m} r_{N}\left[L_{k-1}^{-}, L_{k}^{-}\right]^{-1} L_{m}^{-}\right) \\
& =\left[\begin{array}{c}
u_{j, k-1} \\
u_{j, k}
\end{array}\right]+\sum_{m=k+1}^{\infty}\left(u_{j, m} V_{1, m}+\sum_{l=0}^{N-1} u_{j+l, m} r_{l} V_{2, m}\right)
\end{aligned}
$$

where $r_{N}=Q^{N}\left(I_{2}-Q^{N}\right)^{-1}=r_{0}-I_{2}$ is used in the third equality. Therefore, (23) is proven. The proof of (24) is given in Lemmas 3.2, 3.4, 3.8, 3.9 in [10].

If $h_{j}=h_{j+1}$, denote

$$
\mathcal{U}_{j}=\left[\begin{array}{c}
\dot{u}_{j, k-1}-u_{j, k-1} \\
\dot{u}_{j, k}-u_{j, k}
\end{array}\right]-\left[\begin{array}{c}
\dot{u}_{j+1, k-1}-u_{j+1, k-1} \\
\dot{u}_{j+1, k}-u_{j+1, k}
\end{array}\right]
$$

then by (22) and (23), we have

$$
\begin{aligned}
& \mathcal{U}_{j}=\sum_{m=k+1}^{\infty}\left(u_{j, m}-u_{j+1, m}\right) \mathcal{M}_{m}, \quad \text { if A1, } \\
& \mathcal{U}_{j}=\sum_{m=k+1}^{\infty}\left(\left(u_{j, m}-u_{j, m}\right) V_{1, m}+\sum_{l=0}^{N-1}\left(u_{j+l, m}-u_{j+l+1, m}\right) r_{l} V_{2, m}\right), \text { if A2/A3. }
\end{aligned}
$$

When assumption A1 is satisfied (25) is a direct result of (69).

When assumption A2 is satisfied, we have

$$
\left\|\mathcal{U}_{j}\right\|_{\infty} \leq C\left(1+\sum_{l=0}^{N-1}\left\|r_{l}\right\|_{\infty}\right) \max _{j+l \in \mathbb{Z}_{N}} h^{k+2}\left|u^{(k+2)}\left(x_{j+l-\frac{1}{2}}\right)\right| \leq C h^{k+2}|u|_{W^{k+2, \infty}(I)}
$$

where (66), (69) and the fact that $V_{1, m}, V_{2, m}, \forall m \geq 0$ are constant matrices independent of $h$ are used in above inequalities.

When assumption A3 is satisfied, we perform more detailed computation of $\mathcal{S}_{j}$ and use Fourier analysis to bound it by utilizing the smoothness and periodicity. If $u \in W^{k+2+n, \infty}(I)$,

$$
\begin{aligned}
\mathcal{U}_{j} & =\sum_{l=0}^{N-1} r_{l} \sum_{m=k+1}^{\infty}\left(u_{l+j, m}-u_{l+j+1, m}+\sum_{s=1}^{n} \mu_{s} h_{j}^{k+1+s} u^{(k+1+s)}\left(x_{j+l-\frac{1}{2}}\right)\right) V_{2, m} \\
& -\sum_{l=0}^{N-1} r_{l} \sum_{m=k+1}^{\infty} \sum_{s=1}^{n} \mu_{s} h_{j}^{k+1+s} u^{(k+1+s)}\left(x_{j+l-\frac{1}{2}}\right) V_{2, m}+\sum_{m=k+1}^{\infty}\left(u_{j, m}-u_{j, m}\right) V_{1, m} .
\end{aligned}
$$


When $\frac{|\Gamma|}{|\Lambda|}<1, Q=-A^{-1} B$ has two imaginary eigenvalues $\lambda_{1}, \lambda_{2}$ with $\left|\lambda_{1}\right|=\left|\lambda_{2}\right|=1$. By (59) of [10], we have $r_{l}=\frac{\lambda_{1}^{l}}{1-\lambda_{1}^{N}} Q_{1}+\frac{\lambda_{2}^{l}}{1-\lambda_{2}^{N}}\left(I_{2}-Q_{1}\right)$, where $Q_{1}$ is a constant matrix independent of $h$, and defined in (60) and (61) in [10].

Thus, by (69) and (70)

$$
\begin{aligned}
\left\|\mathcal{U}_{j}\right\|_{\infty} & \leq C \sum_{l=0}^{N-1}\left\|r_{l}\right\|_{\infty} h^{k+2+n}|u|_{W^{k+2+n, \infty}(I)}+C h^{k+2}|u|_{W^{k+2, \infty}(I)} \\
& +\left\|\sum_{m=k+1}^{\infty} \sum_{s=1}^{n} \mu_{s} h^{k+1+s}\left(Q_{1} \bigotimes_{\lambda_{1}}+\left(I_{2}-Q_{1}\right) \bigotimes_{\lambda_{2}}\right) u^{(k+1+s)}\left(x_{j-\frac{1}{2}}\right) V_{2, m}\right\|_{\infty} .
\end{aligned}
$$

By (72), we have

$$
\left\|\mathcal{U}_{j}\right\|_{\infty} \leq C_{1} h^{k+2}
$$

When $\frac{|\Gamma|}{|\Lambda|}=1, Q=-A^{-1} B$ has two repeated eigenvalues. By (71) of [10], we have $r_{l}=\frac{(-1)^{l}}{2} I_{2}+(-1)^{l} \frac{-N+2 l}{4 \Gamma} Q_{2}$, where $Q_{2} / \Gamma$ is a constant matrix, we estimate $\mathcal{U}_{j}$ by the same procedure as previous case and obtain

$$
\begin{aligned}
\left\|\mathcal{U}_{j}\right\|_{\infty} & \leq C \sum_{l=0}^{N-1}\left\|r_{l}\right\|_{\infty} h^{k+2+n}|u|_{W^{k+4, \infty}(I)}+C h^{k+2}|u|_{W^{k+2, \infty}(I)} \\
& +\left\|\sum_{m=k+1}^{\infty} \sum_{s=1}^{n} \mu_{s} h^{k+1+s} \frac{1}{2}\left(\bigotimes_{-1}+\frac{Q_{2}}{\Gamma} \boxplus\right) u^{(k+1+n)}\left(x_{j-\frac{1}{2}}\right) V_{2, m}\right\|_{\infty} \leq C_{1} h^{k+2} .
\end{aligned}
$$

Finally, the estimates for $\mathcal{U}_{j}$ is complete for all assumptions and (25) is proven.

\section{A.4 Proof of Lemma 3.4}

Proof. By the definition of $P_{h}^{\dagger}$, the solution of $\grave{u}_{j, k-1}, \grave{u}_{j, k}$ has similar linear algebraic system as (22). That is, under assumption A2 or A3, the existence and uniqueness condition is $\operatorname{det}(A+B)=2\left((-1)^{k} \Gamma+\Lambda\right) \neq 0$. Thus,

$$
\left[\begin{array}{c}
\grave{u}_{j, k-1} \\
\grave{u}_{j, k}
\end{array}\right]=\left[\begin{array}{c}
u_{j, k-1} \\
u_{j, k}
\end{array}\right]+\sum_{m=k+1}^{\infty} u_{j, m} \mathcal{M}_{m} .
$$

And then, by (19) and (10), (28) is proven.

If any of the assumptions A2/A3 is satisfied, then the difference can be written as

$$
\left.W u\right|_{I_{j}}=\left.P_{h}^{\star} u\right|_{I_{j}}-\left.P_{h}^{\dagger} u\right|_{I_{j}}=\left(\dot{u}_{j, k-1}-\grave{u}_{j, k-1}\right) L_{j, k-1}+\left(\dot{u}_{j, k}-\grave{u}_{j, k}\right) L_{j, k} .
$$

The properties of $P_{h}^{\star} u$ and $P_{h}^{\dagger} u$ yield the following coupled system

$$
\begin{gathered}
A\left[\begin{array}{c}
\dot{u}_{j, k-1}-\grave{u}_{j, k-1} \\
\dot{u}_{j, k}-\grave{u}_{j, k}
\end{array}\right]+B\left[\begin{array}{c}
\dot{u}_{j+1, k-1}-\grave{u}_{j+1, k-1} \\
\dot{u}_{j+1, k}-\grave{u}_{j+1, k}
\end{array}\right]=\left[\begin{array}{c}
\tau_{j} \\
\iota_{j}
\end{array}\right], \quad \forall j \in \mathbb{Z}_{N}, \\
{\left[\begin{array}{c}
\tau_{j} \\
\iota_{j}
\end{array}\right]=\left.\left[\begin{array}{c}
u \\
u_{x}
\end{array}\right]\right|_{x_{j+\frac{1}{2}}}-\left.G\left[\begin{array}{c}
P_{h}^{\dagger} u \\
\left(P_{h}^{\dagger} u\right)_{x}
\end{array}\right]\right|_{x_{j+\frac{1}{2}}} ^{-}-\left.H\left[\begin{array}{c}
P_{h}^{\dagger} u \\
\left(P_{h}^{\dagger} u\right)_{x}
\end{array}\right]\right|_{x_{j+\frac{1}{2}}} ^{+}=G\left[\begin{array}{c}
\left.\left(u-P_{h}^{\dagger} u\right)\right|_{x_{j+\frac{1}{2}}} ^{-}-\left.\left(u-P_{h}^{\dagger} u\right)\right|_{x_{j+\frac{3}{2}}} ^{-} \\
\left.\left(u-P_{h}^{\dagger} u\right)_{x}\right|_{x_{j+\frac{1}{2}}} ^{-}-\left.\left(u-P_{h}^{\dagger} u\right)_{x}\right|_{x_{j+\frac{3}{2}}} ^{-}
\end{array}\right],}
\end{gathered}
$$


where the second equality was obtained by the definition of $P_{h}^{\dagger} u(27 \mathrm{~b})$.

Gather the relations above for all $j$ results in a large $2 N \times 2 N$ linear system with block circulant matrix $M$, defined in (15), as coefficient matrix, then the solution is

$$
\left[\begin{array}{c}
\dot{u}_{j, k-1}-\grave{u}_{j, k-1} \\
\dot{u}_{j, k}-\grave{u}_{j, k}
\end{array}\right]=\sum_{l=0}^{N-1} r_{l} A^{-1}\left[\begin{array}{c}
\tau_{l+j} \\
\iota_{l+j}
\end{array}\right], \quad j \in \mathbb{Z}_{N}
$$

where by periodicity, when $l+j>N, \tau_{l+j}=\tau_{l+j-N}, \iota_{l+j}=\iota_{l+j-N}$.

On uniform mesh, by the definition of $R_{j, m}$ in (31), $R_{j, m}(1)$ and $\left(R_{j, m}\right)_{x}(1)$ are independent of $j$, we denote the corresponding values as $R_{m}(1)$ and $\left(R_{m}\right)_{x}(1)$ and let $R_{m}^{-}=$ $\left[R_{m}(1),\left(R_{m}\right)_{x}(1)\right]^{T}$. By (30), we have

$$
\left[\begin{array}{c}
\left.\left(u-P_{h}^{\dagger} u\right)\right|_{x_{j+\frac{1}{2}}} ^{-}-\left.\left(u-P_{h}^{\dagger} u\right)\right|_{x_{j+\frac{3}{2}}} \\
\left.\left(u-P_{h}^{\dagger} u\right)_{x}\right|_{\bar{x}_{j+\frac{1}{2}}} ^{-}-\left.\left(u-P_{h}^{\dagger} u\right)_{x}\right|_{x_{j+\frac{3}{2}}} ^{-}
\end{array}\right]=\sum_{m=k+1}^{\infty}\left(u_{j, m}-u_{j+1, m}\right) R_{m}^{-}
$$

and

$$
\left[\begin{array}{c}
\dot{u}_{j, k-1}-\grave{u}_{j, k-1} \\
\dot{u}_{j, k}-\grave{u}_{j, k}
\end{array}\right]=\sum_{l=0}^{N-1} r_{l}\left(\sum_{m=k+1}^{\infty}\left(u_{l+j, m}-u_{l+j+1, m}\right) A^{-1} G R_{m}^{-}\right) \doteq \mathcal{S}_{j}, \quad j \in \mathbb{Z}_{N} .
$$

We can estimate $\mathcal{S}_{j}$ by the same lines as the estimation of $\mathcal{U}_{j}$ in Appendix A.3 and (29) is proven.

\section{A.5 Proof of Lemma 4.1}

Proof. By error equation, the symmetry of $A(\cdot, \cdot)$ and the definition of $s_{h}$, we have

$$
0=a\left(e, v_{h}\right)=a\left(\epsilon_{h}, v_{h}\right)+a\left(\zeta_{h}, v_{h}\right)=\int_{I} s_{h} v_{h} d x+\int_{I}\left(\zeta_{h}\right)_{t} v_{h} d x-i A\left(v_{h}, \zeta_{h}\right), \quad \forall v_{h} \in V_{h}^{k} .
$$

Now, we are going to choose three special test functions to extract superconvergence properties (35)-(37) about $\zeta_{h}$. We first prove (35). Due to the invertibility of the coefficient matrix $M$, there exists a nontrivial function $v_{1} \in V_{h}^{k}$, such that $\forall j \in \mathbb{Z}_{N},\left.v_{1}\right|_{I_{j}}=$ $\alpha_{j, k-1} L_{j, k-1}+\alpha_{j, k} L_{j, k}+\overline{\left(\zeta_{h}\right)_{x x}}, \int_{I_{j}} v_{1}\left(\zeta_{h}\right)_{x x} d x=\left\|\left(\zeta_{h}\right)_{x x}\right\|_{L^{2}\left(I_{j}\right)}^{2},\left.\hat{v}_{1}\right|_{j+\frac{1}{2}}=0$ and $\left.\widetilde{\left(v_{1}\right)_{x}}\right|_{j+\frac{1}{2}}=0$. Thus $A\left(v_{h}, \zeta_{h}\right)=\left\|\left(\zeta_{h}\right)_{x x}\right\|^{2}$. Let $v_{h}=v_{1}$, then (76) becomes

$$
0=\int_{I} s_{h} v_{1} d x+\int_{I}\left(\zeta_{h}\right)_{t} v_{1} d x-i\left\|\left(\zeta_{h}\right)_{x x}\right\|^{2}
$$

Hence $\left\|\left(\zeta_{h}\right)_{x x}\right\|^{2} \leq\left\|s_{h}+\left(\zeta_{h}\right)_{t}\right\| \cdot\left\|v_{1}\right\|$. In order to show the estimates for $\left\|\left(\zeta_{h}\right)_{x x}\right\|$, it remains to estimate $\left\|v_{1}\right\|$.

When the assumption A1 holds, the definition of $v_{1}$ yields the following local system for each pair of $\alpha_{j, k-1}$ and $\alpha_{j, k}$,

$$
\left(A_{j}+B_{j}\right)\left[\begin{array}{c}
\alpha_{j, k-1} \\
\alpha_{j, k}
\end{array}\right]=-\left.G\left[\overline{\frac{\left(\zeta_{h}\right)_{x x}^{-}}{\left(\zeta_{h}\right)_{x x x}^{-}}}\right]\right|_{j+\frac{1}{2}}-\left.H\left[\overline{\frac{\left(\zeta_{h}\right)_{x x}^{+}}{\left(\zeta_{h}\right)_{x x x}^{+}}}\right]\right|_{j-\frac{1}{2}}, \quad \forall j \in \mathbb{Z}_{N} .
$$


By simple algebra

$$
\left[\begin{array}{c}
\alpha_{j, k-1} \\
\alpha_{j, k}
\end{array}\right]=-\left.\left(A_{j}+B_{j}\right)^{-1} G\left[\begin{array}{cc}
1 & 0 \\
0 & \frac{1}{h_{j}}
\end{array}\right]\left[\overline{\frac{\left(\zeta_{h}\right)_{x x}^{-}}{h_{j}\left(\zeta_{h}\right)_{x x x}^{-}}}\right]\right|_{j+\frac{1}{2}}-\left.\left(A_{j}+B_{j}\right)^{-1} H\left[\begin{array}{cc}
1 & 0 \\
0 & \frac{1}{h_{j}}
\end{array}\right]\left[\overline{\frac{\left(\zeta_{h}\right)_{x x}^{+}}{h_{j}\left(\zeta_{h}\right)_{x x x}^{+}}}\right]\right|_{j-\frac{1}{2}},
$$

By orthogonality of Legendre polynomials, it follows that

$$
\begin{aligned}
\left\|v_{1}\right\|_{L^{2}\left(I_{j}\right)}^{2} & =\left|\alpha_{j, k-1}\right|^{2} \int_{I_{j}} L_{j, k-1}^{2} d x+\left|\alpha_{j, k}\right|^{2} \int_{I_{j}} L_{j, k}^{2} d x+\left\|\left(\zeta_{h}\right)_{x x}\right\|_{L^{2}\left(I_{j}\right)}^{2} \\
& \leq C\left(h_{j}\left\|\left(\zeta_{h}\right)_{x x}\right\|_{L^{2}\left(\partial I_{j}\right)}^{2}+h_{j}^{3}\left\|\left(\zeta_{h}\right)_{x x x}\right\|_{L^{2}\left(\partial I_{j}\right)}^{2}+\left\|\left(\zeta_{h}\right)_{x x}\right\|_{L^{2}\left(I_{j}\right)}^{2}\right) \leq C\left\|\left(\zeta_{h}\right)_{x x}\right\|_{L^{2}\left(I_{j}\right)}^{2},
\end{aligned}
$$

where Lemma 3.1, trace inequalities and inverse inequalities are used in above inequality. Therefore, (35) is proven when assumption A1 is satisfied.

Similarly, we define $v_{2} \in V_{h}^{k}$, such that $\forall j \in \mathbb{Z}_{N},\left.v_{2}\right|_{I_{j}}=\alpha_{j, k-1} L_{j, k-1}+\alpha_{j, k} L_{j, k}, \int_{I_{j}} v_{2}\left(\zeta_{h}\right)_{x x} d x=$ $0,\left.\hat{v}_{2}\right|_{j+\frac{1}{2}}=0$ and $\left.\widetilde{\left(v_{2}\right)_{x}}\right|_{j+\frac{1}{2}}=\left.\overline{\left[\zeta_{h}\right]}\right|_{j+\frac{1}{2}}$. Thus $A\left(v_{h}, \zeta_{h}\right)=-\sum_{j=1}^{N} \mid\left[\zeta_{h}\right]_{j+\frac{1}{2}}^{2}$. When assumption A1 is satisfied, this definition yields the following local system for each pair of $\alpha_{j, k-1}$ and $\alpha_{j, k}$

$$
\left(A_{j}+B_{j}\right)\left[\begin{array}{c}
\alpha_{j, k-1} \\
\alpha_{j, k}
\end{array}\right]=\left.G\left[\frac{0}{\left[\zeta_{h}\right]}\right]\right|_{j+\frac{1}{2}}+\left.H\left[\frac{0}{\left[\zeta_{h}\right]}\right]\right|_{j-\frac{1}{2}}, \quad \forall j \in \mathbb{Z}_{N} .
$$

By same algebra as above, we have

$$
\left[\begin{array}{c}
\alpha_{j, k-1} \\
\alpha_{j, k}
\end{array}\right]=\left.\left(A_{j}+B_{j}\right)^{-1} G\left[\begin{array}{cc}
1 & 0 \\
0 & \frac{1}{h_{j}}
\end{array}\right]\left[\begin{array}{c}
0 \\
h_{j} \overline{\left[\zeta_{h}\right]}
\end{array}\right]\right|_{j+\frac{1}{2}}+\left.\left(A_{j}+B_{j}\right)^{-1} H\left[\begin{array}{cc}
1 & 0 \\
0 & \frac{1}{h_{j}}
\end{array}\right]\left[\begin{array}{c}
0 \\
h_{j}\left[\zeta_{h}\right]
\end{array}\right]\right|_{j-\frac{1}{2}} .
$$

By Lemma 3.1, it follows directly that

$$
\left\|v_{2}\right\|_{L^{2}\left(I_{j}\right)}^{2} \leq C h_{j}^{3}\left(\left|\left[\zeta_{h}\right]\right|_{j+\frac{1}{2}}^{2}+\left|\left[\zeta_{h}\right]\right|_{j-\frac{1}{2}}^{2}\right) .
$$

Plug $v_{2}$ in (76), we obtain

$$
\sum_{j=1}^{N}\left|\left[\zeta_{h}\right]\right|_{j+\frac{1}{2}}^{2}=i \int_{I} s_{h} v_{2} d x+i \int_{I}\left(\zeta_{h}\right)_{t} v_{2} d x \leq\left\|s_{h}+\left(\zeta_{h}\right)_{t}\right\|\left\|v_{2}\right\| .
$$

Therefore, (36) is proven when assumption A1 is satisfied.

Finally, we can also choose $v_{3} \in V_{h}^{k}$, such that $\forall j \in \mathbb{Z}_{N},\left.v_{3}\right|_{I_{j}}=\alpha_{j, k-1} L_{j, k-1}+\alpha_{j, k} L_{j, k}$ such that $\int_{I_{j}} v_{3}\left(\zeta_{h}\right)_{x x} d x=0,\left.\hat{v}_{3}\right|_{j+\frac{1}{2}}=\left.\overline{\left[\left(\zeta_{h}\right)_{x}\right]}\right|_{j+\frac{1}{2}}$ and $\left.\widetilde{\left(v_{3}\right)_{x}}\right|_{j+\frac{1}{2}}=0$. Thus $A\left(v_{h}, \zeta_{h}\right)=$ $\sum_{j=1}^{N}\left|\left[\left(\zeta_{h}\right)_{x}\right]\right|_{j+\frac{1}{2}}^{2}$. Follow the same lines as the estimates for $v_{2}$, we end up with the estimates

$$
\left\|v_{3}\right\|_{L^{2}\left(I_{j}\right)}^{2} \leq C h_{j}\left(\left|\left[\left(\zeta_{h}\right)_{x}\right]\right|_{j+\frac{1}{2}}^{2}+\left|\left[\left(\zeta_{h}\right)_{x}\right]\right|_{j-\frac{1}{2}}^{2}\right) .
$$

Plug $v_{3}$ in (76), we obtain (37) when assumption A1 is satisfied.

Under assumption A2, we need to compute $\sum_{j=1}^{N}\left(\left|\alpha_{j, k-1}\right|^{2}+\left|\alpha_{j, k}\right|^{2}\right)$ to estimate $\left\|v_{1}\right\|^{2}$. The definition of $v_{1}$ yields the following coupled system

$$
A\left[\begin{array}{c}
\alpha_{j, k-1} \\
\alpha_{j, k}
\end{array}\right]+B\left[\begin{array}{c}
\alpha_{j+1, k-1} \\
\alpha_{j+1, k}
\end{array}\right]=-\left.G\left[\overline{\frac{\left(\zeta_{h}\right)_{x x}^{-}}{\left(\zeta_{h}\right)_{x x x}^{-}}}\right]\right|_{j+\frac{1}{2}}-\left.H\left[\overline{\frac{\left(\zeta_{h}\right)_{x x}^{+}}{\left(\zeta_{h}\right)_{x x x}^{+}}}\right]\right|_{j+\frac{1}{2}}, \quad j \in \mathbb{Z}_{N} .
$$


Write it in matrix form

$$
M \boldsymbol{\alpha}=\boldsymbol{b}, \quad \boldsymbol{\alpha}=\left[\boldsymbol{\alpha}_{1}, \cdots, \boldsymbol{\alpha}_{N}\right]^{T},
$$

where $M$ is defined in (15) and

$$
\boldsymbol{\alpha}_{j}=\left[\alpha_{j, k-1}, \alpha_{j, k}\right], \boldsymbol{b}=\left[\boldsymbol{b}_{1}, \cdots, \boldsymbol{b}_{N}\right]^{T}, \boldsymbol{b}_{j}=-\left.G\left[\overline{\frac{\left(\zeta_{h}\right)_{x x}^{-}}{\left(\zeta_{h}\right)_{x x x}^{-}}}\right]\right|_{j+\frac{1}{2}}-\left.H\left[\frac{\overline{\left(\zeta_{h}\right)_{x x}^{+}}}{\left(\zeta_{h}\right)_{x x x}^{+}}\right]\right|_{j+\frac{1}{2}} .
$$

Multiply $A^{-1}$ from the left in (78), we get an equivalent system

$$
M^{\prime} \boldsymbol{\alpha}=\boldsymbol{b}^{\prime}, \quad M^{\prime}=\operatorname{circ}\left(I_{2}, A^{-1} B, 0_{2}, \cdots, 0_{2}\right), \boldsymbol{b}^{\prime}=\left[\boldsymbol{b}_{1}^{\prime}, \cdots, \boldsymbol{b}_{N}^{\prime}\right]^{T}, \boldsymbol{b}_{j}^{\prime}=A^{-1} \boldsymbol{b}_{j},
$$

and $\left(M^{\prime}\right)^{-1}=\operatorname{circ}\left(r_{0}, \cdots, r_{N-1}\right)$. By Theorem 5.6.4 in [15] and similar to the proof in Lemma 3.1 in [6],

$$
M^{\prime}=\left(\mathcal{F}_{N}^{*} \otimes I_{2}\right) \Omega\left(\mathcal{F}_{N} \otimes I_{2}\right),
$$

where $\mathcal{F}_{N}$ is the $N \times N$ discrete Fourier transform matrix defined by $\left(\mathcal{F}_{N}\right)_{i j}=\frac{1}{\sqrt{N}} \bar{\omega}^{(i-1)(j-1)}, \omega=$ $e^{i \frac{2 \pi}{N}} . \mathcal{F}_{N}$ is symmetric and unitary and

$$
\boldsymbol{\Omega}=\operatorname{diag}\left(I_{2}+A^{-1} B, I_{2}+\omega A^{-1} B, \cdots, I_{2}+\omega^{N-1} A^{-1} B\right) .
$$

The assumption $\frac{|\Gamma|}{|\Lambda|}>1$ in A2 ensures that the eigenvalues of $Q=-A^{-1} B$ (see (52) in [10]) are not 1 , thus $I_{2}+\omega^{j} A^{-1} B, \forall j$, is nonsingular and $\Omega$ is invertible. Then

$$
\left|\rho\left(\left(M^{\prime}\right)^{-1}\right)\right|=\left\|\left(M^{\prime}\right)^{-1}\right\|_{2} \leq\left\|\mathcal{F}_{N}^{*} \otimes I_{2}\right\|_{2}\|\Omega\|_{2}\left\|\mathcal{F}_{N} \otimes I_{2}\right\|_{2} \leq C .
$$

Therefore,

$$
\sum_{j=1}^{N}\left(\left|\alpha_{j, k-1}\right|^{2}+\left|\alpha_{j, k}\right|^{2}\right)=\boldsymbol{\alpha}^{T} \boldsymbol{\alpha}=\left(\boldsymbol{b}^{\prime}\right)^{T}\left(M^{\prime}\right)^{-T}\left(M^{\prime}\right)^{-1}\left(\boldsymbol{b}^{\prime}\right)^{T} \leq\left\|\left(M^{\prime}\right)^{-1}\right\|_{2}^{2}\left\|\boldsymbol{b}^{\prime}\right\|_{2}^{2} \leq C \sum_{j=1}^{N}\left\|\boldsymbol{b}_{j}^{\prime}\right\|_{2}^{2} .
$$

Since $A^{-1} G\left[\begin{array}{cc}1 & 0 \\ 0 & \frac{1}{h}\end{array}\right], A^{-1} H\left[\begin{array}{cc}1 & 0 \\ 0 & \frac{1}{h}\end{array}\right]$ are constant matrices, we have

$$
\begin{aligned}
\left\|\boldsymbol{b}_{j}^{\prime}\right\|_{2}^{2} & \leq C\left(\left.\left\|\left.\left[\frac{\overline{\left(\zeta_{h}\right)_{x x}^{-}}}{h \overline{\left(\zeta_{h}\right)_{x x x}^{-}}}\right]\right|_{j+\frac{1}{2}}\right\|\left\|_{2}+\right\|\left[\frac{\overline{\left(\zeta_{h}\right)_{x x}^{+}}}{h\left(\overline{\left.\zeta_{h}\right)_{x x x}^{+}}\right.}\right]\right|_{j+\frac{1}{2}}\|\|_{2}\right) \\
& \leq C\left(\left\|\left(\zeta_{h}\right)_{x x}\right\|_{L^{2}\left(\partial I_{j}\right)}^{2}+\left\|\left(\zeta_{h}\right)_{x x}\right\|_{L^{2}\left(\partial I_{j+1}\right)}^{2}+h^{2}\left\|\left(\zeta_{h}\right)_{x x x}\right\|_{L^{2}\left(\partial I_{j}\right)}^{2}+h^{2}\left\|\left(\zeta_{h}\right)_{x x x}\right\|_{L^{2}\left(\partial I_{j+1}\right)}^{2}\right) \\
& \leq C\left(\left\|\left(\zeta_{h}\right)_{x x}\right\|_{L^{2}\left(\partial I_{j}\right)}^{2}+\left\|\left(\zeta_{h}\right)_{x x}\right\|_{L^{2}\left(\partial I_{j+1}\right)}^{2}\right)
\end{aligned}
$$

where inverse inequality is used to obtain the last inequality. Finally, we obtain the estimate

$$
\begin{aligned}
\left\|v_{1}\right\|^{2} & =\sum_{j=1}^{N}\left|\alpha_{j, k-1}\right|^{2}\left\|L_{j, k-1}\right\|_{L^{2}\left(I_{j}\right)}^{2}+\sum_{j=1}^{N}\left|\alpha_{j, k}\right|^{2}\left\|L_{j, k}\right\|_{L^{2}\left(I_{j}\right)}^{2}+\left\|\left(\zeta_{h}\right)_{x x}\right\|^{2} \\
& \leq\left\|\left(\zeta_{h}\right)_{x x}\right\|^{2}+C h \sum_{j=1}^{N}\left(\left|\alpha_{j, k-1}\right|^{2}+\left|\alpha_{j, k}\right|^{2}\right) \\
& \leq\left\|\left(\zeta_{h}\right)_{x x}\right\|^{2}+C h \sum_{j=1}^{N}\left(\left\|\left(\zeta_{h}\right)_{x x}\right\|_{L^{2}\left(\partial I_{j}\right)}^{2}+\left\|\left(\zeta_{h}\right)_{x x}\right\|_{L^{2}\left(\partial I_{j+1}\right)}^{2}\right) \\
& \leq\left\|\left(\zeta_{h}\right)_{x x}\right\|^{2}+C h\left\|\left(\zeta_{h}\right)_{x x}\right\|_{L^{2}\left(\partial \mathcal{I}_{N}\right)}^{2} \leq C\left\|\left(\zeta_{h}\right)_{x x}\right\|^{2}
\end{aligned}
$$


where inverse inequality is used to obtain the last inequality. Then the estimates for (35) hold true. (36) and (37) can be proven by the same procedure when assumption A2 is satisfied, and the steps are omitted for brevity.

Remark A.1. When assumption A3 is satisfied, the eigenvalues of $Q$ are two complex number with magnitude 1, then a constant bound for $\rho\left(\left(M^{\prime}\right)^{-1}\right)$ as in (79) is not possible. Therefore, we cannot obtain similar results for assumption A3.

\section{A.6 Proof for Lemma 4.2}

Proof. Since $w_{q} \in V_{h}^{k}$, we have

$$
\left.w_{q}\right|_{I_{j}}=\sum_{m=0}^{k} c_{j, m}^{q} L_{j, m} .
$$

Let $v_{h}=D^{-2} L_{j, m}, m \leq k-2$ in (38a), we obtain

$$
c_{j, m}^{q}=-i \frac{2 m+1}{h_{j}} \frac{h_{j}^{2}}{4} \int_{I_{j}} \partial_{t} w_{q-1} D^{-2} L_{j, m} d x .
$$

Since $D^{-2} L_{j, m} \in P_{c}^{m+2}\left(I_{j}\right)$, by the property $u-P_{h}^{\star} u \perp V_{h}^{k-2}$ in the $L^{2}$ inner product sense, we have

$$
c_{j, m}^{1}= \begin{cases}-i \frac{2 m+1}{h_{j}} \frac{h_{j}^{2}}{4} \int_{I_{j}} \partial_{t}\left(u-P_{h}^{\star} u\right) D^{-2} L_{j, m} d x=0, & m \leq k-4, \\ -i \frac{2 m+1}{h_{j}} \frac{h_{j}^{2}}{4} \int_{I_{j}} \partial_{t}\left(\left(u_{j, k-1}-\dot{u}_{j, k-1}\right) L_{j, k-1}+\left(u_{j, k}-\dot{u}_{j, k}\right) L_{j, k}\right) D^{-2} L_{j, m} d x, & m=k-3, k-2 .\end{cases}
$$

By induction using (80), (81), (82), for $0 \leq m \leq k-2-2 q, c_{j, m}^{q}=0$.

Furthermore, the first nonzero coefficient can be written in a simpler form related to $u_{j, k-1}$ by induction.

When $q=1$, we compute $c_{j, k-3}^{1}$ by $(\underline{82})$ and the definition of $w_{0}$. That is

$$
\begin{aligned}
c_{j, k-3}^{1} & =-i \frac{2(k-3)+1}{h_{j}}\left(\frac{h_{j}}{2}\right)^{2} \partial_{t}\left(u_{j, k-1}-\dot{u}_{j, k-1}\right) \int_{I_{j}} D^{-2} L_{j, k-3} L_{j, k-1} d x \\
& =C h_{j}^{2} \partial_{t}\left(u_{j, k-1}-\dot{u}_{j, k-1}\right) .
\end{aligned}
$$

Suppose $c_{k+1-2 q}^{q-1}=C h_{j}^{2 q-2} \partial_{t}^{q-1}\left(u_{j, k-1}-\dot{u}_{j, k-1}\right)$, then

$$
\begin{aligned}
c_{j, k-1-2 q}^{q} & =-i \frac{2(k-1-2 q)+1}{h_{j}}\left(\frac{h_{j}}{2}\right)^{2} \partial_{t} c_{j, k+1-2 q}^{q-1} \int_{I_{j}} D^{-2} L_{j, k-1-2 q} L_{j, k+1-2 q} d x \\
& =C h_{j}{ }^{2 q} \partial_{t}^{q}\left(u_{j, k-1}-\dot{u}_{j, k-1}\right) .
\end{aligned}
$$

The induction is completed and (42) is proven when $r=0$.

Next, we begin estimating the coefficient $c_{j, m}^{q}$. By Holder's inequality and (81), we have the estimates for $c_{j, m}^{q}, k-1-2 q \leq m \leq k-2$,

$$
\left|c_{j, m}^{q}\right| \leq C h^{2-\frac{1}{s}}\left\|\partial_{t} w_{q-1}\right\|_{L^{s}\left(I_{j}\right)} .
$$


To estimate the coefficients $c_{j, k-1}^{q}, c_{j, k}^{q}$, we need to discuss it by cases. If assumption A1 is satisfied, meaning (38b) and (38c) can be decoupled and therefore $w_{q}$ is locally defined by (38). By (39) and following the same algebra of solving the $k$-th and $(k+1)$-th coefficients in (73),

$$
\left[\begin{array}{c}
c_{j, k-1}^{q} \\
c_{j, k}^{q}
\end{array}\right]=-\sum_{m=0}^{k-2} \mathcal{M}_{j, m} c_{j, m}^{q}
$$

By (19), for all $j \in \mathbb{Z}_{N}$,

$$
\begin{gathered}
\left|c_{j, k-1}^{q}\right|^{2}+\left|c_{j, k}^{q}\right|^{2} \leq C \sum_{m=k-2 q-3}^{k-2}\left|c_{j, m}^{q}\right|^{2} \leq C h^{3}\left\|\partial_{t} w_{q-1}\right\|_{L^{2}\left(I_{j}\right)}^{2} . \\
\max \left(\left|c_{j, k-1}^{q}\right|,\left|c_{j, k}^{q}\right|\right) \leq C \max _{k-2 q-3 \leq m \leq k-2}\left|c_{j, m}^{q}\right| \leq C h^{2}\left\|\partial_{t} w_{q-1}\right\|_{L^{\infty}\left(I_{j}\right)}
\end{gathered}
$$

If one of assumption A2/A3 is satisfied, (39) defines a coupled system. From the same lines for obtaining (23) in Appendix A.3, the solution for $c_{j, k-1}^{q}, c_{j, k}^{q}$ is

$$
\begin{aligned}
{\left[\begin{array}{c}
c_{j, k-1}^{q} \\
c_{j, k}^{q}
\end{array}\right] } & =-\sum_{m=k-1-2 q}^{k-2} \sum_{l=0}^{N-1} r_{l} A^{-1}\left(G L_{m}^{-} c_{j+l, m}^{q}+H L_{m}^{+} c_{j+l+1, m}^{q}\right) \\
& =-\sum_{m=k-1-2 q}^{k-2}\left(c_{j, m}^{q} V_{1, m}+\sum_{l=0}^{N-1} c_{j+l, m}^{q} r_{l} V_{2, m}\right) .
\end{aligned}
$$

Under assumption A2, we can estimate $c_{j, m}^{q}, m=k-1, k$, using (66), that is

$$
\left\|\left[\begin{array}{c}
c_{j, k-1}^{q} \\
c_{j, k}^{q}
\end{array}\right]\right\|_{\infty} \leq C\left(1+\sum_{l=0}^{N-1}\left\|r_{l}\right\|_{\infty}\right) \max \left|c_{j+l, m}^{q}\right| \leq C h^{2}\left\|\partial_{t} w_{q-1}\right\|_{L^{\infty}\left(\mathcal{I}_{N}\right)} .
$$

Under assumption A3, $\sum_{l=0}^{N-1}\left\|r_{l}\right\|_{\infty}$ is unbounded. Thus we use Fourier analysis to bound the coefficients utilizing the smoothness and periodicity by similar idea in [10]. In the rest of the proof, we make use of two operators $\otimes$ and $\boxplus$, which are defined in (71a) and (71b).

When $\frac{|\Gamma|}{|\Lambda|}<1, Q=-A^{-1} B$ has two imaginary eigenvalues $\lambda_{1}, \lambda_{2}$ with $\left|\lambda_{1}\right|=\left|\lambda_{2}\right|=1$. By (59) of [10], we have $r_{l}=\frac{\lambda_{1}^{l}}{1-\lambda_{1}^{N}} Q_{1}+\frac{\lambda_{2}^{l}}{1-\lambda_{2}^{N}}\left(I_{2}-Q_{1}\right)$, where $Q_{1}$ is a constant matrix independent of $h$, and defined in (60) and (61) in [10]. We perform more detailed computation of the 
coefficients. In (82), plug in (23), for $m=k-3, k-2$, when $u_{t} \in W^{k+2+n, \infty}(I)$,

$$
\begin{aligned}
c_{j, m}^{1} & =i \frac{2 m+1}{h} \frac{h^{2}}{4} \int_{I_{j}}\left[L_{j, k-1}, L_{j, k}\right] \partial_{t} \sum_{p=k+1}^{\infty}\left(u_{j, p} V_{1, p}+\sum_{l=0}^{N-1} u_{j+l, p} r_{l} V_{2, p}\right) D^{-2} L_{j, m} d x \\
& =i \frac{2 m+1}{2} \frac{h^{2}}{4} \sum_{p=k+1}^{\infty} \partial_{t}\left(u_{j, p} F_{p, m}^{1}+\sum_{l=0}^{N-1} u_{j+l, p} r_{l} F_{p, m}^{2}\right) \\
& =i \frac{2 m+1}{8} h^{2} \sum_{p=k+1}^{\infty}\left(\sum_{s=0}^{n} \mu_{s} h^{k+1+s} u_{t}^{(k+1+s)}\left(x_{j-\frac{1}{2}}\right) F_{p, m}^{1}\right. \\
& \left.+\sum_{l=0}^{N-1}\left(\frac{\lambda_{1}^{l}}{1-\lambda_{1}^{N}} Q_{1}+\frac{\lambda_{2}^{l}}{1-\lambda_{2}^{N}}\left(I_{2}-Q_{1}\right)\right) \sum_{s=0}^{n} \mu_{s} h^{k+1+s} u_{t}^{(k+1+s)}\left(x_{j+l-\frac{1}{2}}\right) F_{p, m}^{2}+O\left(h^{k+n+1}\left|u_{t}\right|_{W^{k+2+n, \infty}(I)}\right)\right) \\
& =i \frac{2 m+1}{8} h^{2} \sum_{p=k+1}^{\infty} \sum_{s=0}^{n} \mu_{s} h^{k+1+s}\left(u_{t}^{(k+1+s)}\left(x_{j-\frac{1}{2}}\right) F_{p, m}^{1}\right. \\
& \left.+\left(Q_{1} \otimes_{\lambda_{1}}+\left(I_{2}-Q_{1}\right) \bigotimes_{\lambda_{2}}\right) u_{t}^{(k+1+s)}\left(x_{j-\frac{1}{2}}\right) F_{p, m}^{2}\right)+O\left(h^{k+3+n}\left|u_{t}\right|_{W^{k+2+n, \infty}(I)}\right),
\end{aligned}
$$

where $F_{p, m}^{\nu}=\frac{2}{h} \int_{I_{j}}\left[L_{j, k-1}, L_{j, k}\right] V_{\nu, p} D^{-2} L_{j, m} d x, \nu=1,2$, are constants independent of $h$ and (68) is used in the third equality.

Plug the formula above into (83), by similar computation, we have

$$
\begin{aligned}
{\left[\begin{array}{c}
c_{j, k-1}^{1} \\
c_{j, k}^{1}
\end{array}\right] } & =-i \frac{2 m+1}{8} h^{2} \sum_{m=k-3}^{k-2} \sum_{p=k+1}^{\infty} \sum_{s=0}^{n} \mu_{s} h^{k+1+s}\left(u_{t}^{(k+1+s)}\left(x_{j-\frac{1}{2}}\right) F_{p, m}^{1} V_{1, m}\right. \\
& +\left(Q_{1} \bigotimes_{\lambda_{1}}+\left(I_{2}-Q_{1}\right) \bigotimes_{\lambda_{2}}\right) u_{t}^{(k+1+s)}\left(x_{j-\frac{1}{2}}\right)\left(F_{p, m}^{2} V_{1, m}+F_{p, m}^{1} V_{2, m}\right) \\
& \left.+\left(Q_{1} \bigotimes_{\lambda_{1}}+\left(I_{2}-Q_{1}\right) \bigotimes_{\lambda_{2}}\right)^{2} u_{t}^{(k+1+s)}\left(x_{j-\frac{1}{2}}\right) F_{p, m}^{2} V_{2, m}\right)+O\left(h^{k+2+n}\left|u_{t}\right|_{W^{k+2+n, \infty}(I)}\right) .
\end{aligned}
$$

By (72), we have

$$
\left(Q_{1} \bigotimes_{\lambda_{1}}+\left(I_{2}-Q_{1}\right) \bigotimes_{\lambda_{2}}\right)^{\nu} u_{t}^{(k+1+s)}\left(x_{j-\frac{1}{2}}\right) \leq C\left|u_{t}\right|_{W^{k+2+s+\nu, 1}(I)} \leq C|u|_{W^{k+4+s+\nu, 1}(I)} .
$$

Therefore,

$$
\left|c_{j, m}^{1}\right| \leq C_{2} h^{k+3}, \quad m=k-3, k-2, \quad \text { and }\left|c_{j, m}^{1}\right| \leq C_{3} h^{k+3}, \quad m=k-1, k .
$$

By induction and similar computation, we can obtain the formula for $c_{j, m}^{q}$. For brevity, we omit the computation and directly show the estimates

$$
\left|c_{j, m}^{q}\right| \leq C_{3 q} h^{k+1+2 q}, \quad k-1-2 q \leq m \leq k .
$$

When $\frac{|\Gamma|}{|\Lambda|}=1, Q=-A^{-1} B$ has two repeated eigenvalues. By (71) of [10], we have $r_{l}=\frac{(-1)^{l}}{2} I_{2}+(-1)^{l} \frac{-N+2 l}{4 \Gamma} Q_{2}$, where $Q_{2} / \Gamma$ is a constant matrix, then by (23) and (68). For $m=k-3, k-2$, when $u_{t} \in W^{k+2+n, \infty}(I)$, we compute $c_{j, m}^{1}$ by the same procedure as previous case and obtain

$$
\begin{aligned}
c_{j, m}^{1} & =i \frac{2 m+1}{8} h^{2} \sum_{p=k+1}^{\infty} \sum_{s=0}^{n} \mu_{s} h^{k+1+s}\left(u_{t}^{(k+1+s)}\left(x_{j-\frac{1}{2}}\right) F_{p, m}^{1}\right. \\
& \left.+\frac{1}{2}\left(\bigotimes_{-1}+\frac{Q_{2}}{\Gamma} \boxplus\right) u_{t}^{(k+1+s)}\left(x_{j-\frac{1}{2}}\right) F_{p, m}^{2}\right)+O\left(h^{k+3+n}\left|u_{t}\right|_{W^{k+2+n, \infty}(I)}\right) .
\end{aligned}
$$


Plug formula above into (져), we have

$$
\begin{aligned}
{\left[\begin{array}{c}
c_{j, k-1}^{1} c_{j, k}^{1}
\end{array}\right] } & =-i \frac{2 m+1}{8} h^{2} \sum_{m=k-3}^{k-2} \sum_{p=k+1}^{\infty} \sum_{s=0}^{n} \mu_{s} h^{k+1+s}\left(u_{t}^{(k+1+s)}\left(x_{j-\frac{1}{2}}\right) F_{p, m}^{1} V_{1, m}\right. \\
& +\frac{1}{2}\left(\bigotimes_{-1}+\frac{Q_{2}}{\Gamma} \boxplus\right) u_{t}^{(k+1+s)}\left(x_{j-\frac{1}{2}}\right)\left(F_{p, m}^{2} V_{1, m}+F_{p, m}^{1} V_{2, m}\right) \\
& \left.+\frac{1}{4}\left(\bigotimes_{-1}+\frac{Q_{2}}{\Gamma} \boxplus\right)^{2} u_{t}^{(k+1+s)}\left(x_{j-\frac{1}{2}}\right) F_{p, m}^{2} V_{2, m}\right)+O\left(h^{k+2+n}\left|u_{t}\right|_{W^{k+2+n, \infty}(I)}\right) .
\end{aligned}
$$

By (72), we have

$$
\left(\bigotimes_{-1}+\frac{Q_{2}}{\Gamma} \boxplus\right)^{\nu} u_{t}^{(k+1+s)}\left(x_{j-\frac{1}{2}}\right) \leq C\left|u_{t}\right|_{W^{k+2+s+2 \nu, 1}(I)} \leq C|u|_{W^{k+4+s+2 \nu, 1}(I)}
$$

and

$$
\left|c_{j, m}^{1}\right| \leq C_{2} h^{k+3}, \quad m=k-3, k-2, \quad \text { and }\left|c_{j, m}^{1}\right| \leq C_{4} h^{k+3}, \quad m=k-1, k .
$$

By induction and similar computation, we can obtain the formula for $c_{j, m}^{q}$. For brevity, we omit the computation and directly show the estimates

$$
\left|c_{j, m}^{q}\right| \leq C_{4 q} h^{k+1+2 q}, \quad k-1-2 q \leq m \leq k
$$

All the analysis above works when we change definition of $w_{q}$ to $\partial_{t}^{r} w_{q}$ (and change $\left(w_{q-1}\right)_{t}$ to $\partial_{t}^{r+1} w_{q}$ accordingly) in (38). Summarize the estimates for $c_{j, m}^{q}$ under all three assumptions, for $1 \leq q \leq\left\lfloor\frac{k-1}{2}\right\rfloor$, we have

$$
\left|\partial_{t}^{r} c_{j, m}^{q}\right| \leq C_{2 r, q} h^{k+1+2 q}, \quad\left\|\partial_{t}^{r} w_{q}\right\| \leq C\left(\sum_{j=1}^{N} \sum_{m=k-2 q-1}^{k}\left|\partial_{t}^{r} c_{j, m}^{q}\right|^{2} h_{j}\right)^{\frac{1}{2}} \leq C_{2 r, q} h^{k+1+2 q} .
$$

Then (42), (43) is proven. And (44) is a direct result of above estimate and (41). 